Draft VERSiON MARCh 2, 2021

Typeset using $\mathrm{LAT}_{\mathrm{E}} \mathrm{X}$ twocolumn style in AASTeX63

\title{
A new sample of warm extreme debris disks from the ALLWISE catalog
}

\author{
Attila Moór, ${ }^{1,2}$ Péter Ábrahám,,${ }^{1,2}$ Gyula Szabó,${ }^{3,4}$ Krisztián Vida,,${ }^{1,2}$ Gianni Cataldi, ${ }^{5,6}$ Alíz Derekas, ${ }^{3,4}$ \\ Thomas Henning, ${ }^{7}$ Karen Kinemuchi, ${ }^{8}$ Ágnes Kóspál, ${ }^{1,7,2}$ József Kovács, ${ }^{3,4}$ András Pál, ${ }^{1,2}$ Paula Sarkis, ${ }^{7}$ \\ Bálint Seli, ${ }^{1,9}$ Zsófia M. Szabó, ${ }^{9,1}$ and Katalin Takáts ${ }^{10}$ \\ ${ }^{1}$ Konkoly Observatory, Research Centre for Astronomy and Earth Sciences, Eötvös Loránd Research Network (ELKH) \\ Konkoly-Thege Miklós út 15-17, 1121 Budapest, Hungary \\ ${ }^{2}$ ELTE Eötvös Loránd University, Institute of Physics, Pázmány Péter sétány 1/A, 1117 Budapest, Hungary \\ ${ }^{3}$ ELTE Eötvös Loránd University, Gothard Astrophysical Observatory, Szombathely, Hungary \\ ${ }^{4}$ MTA-ELTE Exoplanet Research Group, 9700 Szombathely, Szent Imre h. u. 112, Hungary \\ ${ }^{5}$ National Astronomical Observatory of Japan, Osawa 2-21-1, Mitaka, Tokyo 181-8588, Japan \\ ${ }^{6}$ Department of Astronomy, Graduate School of Science, The University of Tokyo, 7-3-1 Hongo, Bunkyo-ku, Tokyo 113-0033, Japan \\ ${ }^{7}$ Max-Planck-Institut für Astronomie, Königstuhl 17, D-69117 Heidelberg, Germany \\ ${ }^{8}$ Apache Point Observatory and New Mexico State University, Sunspot NM 88349, USA \\ ${ }^{9}$ Eötvös Loránd University, Department of Astronomy, Pázmány Péter sétány 1/A, 1117 Budapest, Hungary \\ ${ }^{10}$ Departamento de Ciencias Físicas, Universidad Andrés Bello, Avda. República 252, 32349 Santiago, Chile
}

\begin{abstract}
Extreme debris disks (EDDs) are rare systems with peculiarly large amounts of warm dust that may stem from recent giant impacts between planetary embryos during the final phases of terrestrial planet growth. Here we report on the identification and characterization of six new EDDs. These disks surround F5-G9 type main-sequence stars with ages $>100 \mathrm{Myr}$, have dust temperatures higher than $300 \mathrm{~K}$ and fractional luminosities between 0.01 and 0.07 . Using time-domain photometric data at 3.4 and $4.6 \mu \mathrm{m}$ from the WISE all sky surveys, we conclude that four of these disks exhibited variable mid-infrared emission between 2010 and 2019. Analyzing the sample of all known EDDs, now expanded to 17 objects, we find that 14 of them showed changes at $3-5 \mu \mathrm{m}$ over the past decade suggesting that mid-infrared variability is an inherent characteristic of EDDs. We also report that wide-orbit pairs are significantly more common in EDD systems than in the normal stellar population. While current models of rocky planet formation predict that the majority of giant collisions occur in the first $100 \mathrm{Myr}$, we find that the sample of EDDs is dominated by systems older than this age. This raises the possibility that the era of giant impacts may be longer than we think, or that some other mechanism(s) can also produce EDDs. We examine a scenario where the observed warm dust stems from the disruption and/or collisions of comets delivered from an outer reservoir into the inner regions, and explore what role the wide companions could play in this process.
\end{abstract}

Keywords: Circumstellar disks(235); Debris disks(363); Exozodiacal dust(500); Extrasolar rocky planets(511); Infrared excess(788); Time domain astronomy(2109)

\section{INTRODUCTION}

Debris disks are comprised of planetesimals and of their erosional products down to micrometer-sized dust particles (Wyatt 2008; Krivov 2010). From this mixture of solids of different sizes, only the smallest particles are observable. The majority of debris disks have been

Corresponding author: Attila Moór

moor@konkoly.hu detected based on the infrared (IR) excess caused by thermal emission from their optically thin dust material. Large far-IR surveys with the Spitzer Space Telescope (Spitzer, Werner et al. 2004) and the Herschel Space Observatory (Pilbratt et al. 2010) indicated that at least $20 \%$ of main-sequence $\mathrm{A}-\mathrm{K}$ type stars are encircled by cold dust ( $\lesssim 100 \mathrm{~K}$ ) implying the presence of debris belts at tens of astronomical units from the central stars (Su et al. 2006; Thureau et al. 2014; Montesinos et al. 2016; Sibthorpe et al. 2018; Hughes et al. 2018). These structures are analogous to the Kuiper-belt of our solar 
system. Their dust particles, which are continuously removed by interactions with the radiation forces of the star and/or with the stellar wind, are thought to be replenished by a collisional cascade involving larger bodies.

Analysis of the spectral energy distribution (SED) implies that a fraction of systems with cold debris dust also host warmer $(>150 \mathrm{~K})$ dust particles, suggesting that an inner debris ring resembling our asteroid belt also exists around the star (Kennedy \& Wyatt 2014; Chen et al. 2014; Ertel et al. 2020). We also know of some systems that possess only warm dust (e.g., Beichman et al. 2011). The majority of these warm dust belts can be explained as a product of long term collisional evolution of a planetesimal belt co-located with the dust (Geiler \& Krivov 2017). Alternatively, or in addition to the in situ dust generation, disrupting comets delivered from an outer reservoir (Bonsor et al. 2012; Nesvorný et al. 2010) or grains transported inward from an outer debris cloud under the influence of PoyntingRobertson and stellar wind drag (Reidemeister et al. 2011; Kennedy \& Piette 2015; Rigley \& Wyatt 2020) can also produce warm dust particles in the inner regions.

The last fifteen years have seen the discovery of several FGK-type main-sequence stars that host unusually dustrich warm debris disks with typical dust temperature of $>300 \mathrm{~K}$ and with fractional luminosity $\left(f_{d}=L_{\text {disk }} / L_{*}\right)$ of $>0.01$. These fractional luminosities are three orders of magnitude higher than those of typical warm debris systems, and suggest that these extreme debris disks (EDDs, Balog et al. 2009) are at least partly optically thick for the stellar illumination. EDDs share some additional common properties. Mid-IR spectroscopic observations revealed that they tend to show strong solid state emission features, implying the presence of small, submicron-sized crystalline dust particles (Rhee et al. 2008; Olofsson et al. 2012; Lisse et al. 2020). Even more uniquely, their photometric monitoring with Spitzer demonstrated that most EDDs display significant variability on monthly to yearly timescales at 3-5 $\mathrm{mm}$ (Meng et al. 2014, 2015; Su et al. 2019b). The ages of known EDDs fall predominantly in the range between 10 and $200 \mathrm{Myr}$, the only exception is BD+20 307 with an age of $>1$ Gyr (Weinberger et al. 2011).

The peculiarly high dust content of EDDs is orders of magnitude above what can be explained by a steady state grinding down process of an in situ planetesimal population, that started when the system was formed (e.g., Wyatt et al. 2007). Instead, the observed properties point to a recent, episodic increase in dust production, which may be related to a giant collision of plan- etary embryos in the inner 1-2 au region (Melis et al. 2010; Su et al. 2019b). Similar collisions - including the Moon-forming event between the proto-Earth and a planetary embryo - are thought to be common during the final accumulation of terrestrial planets in the early ( $\lesssim 100 \mathrm{Myr}$ ) Solar system (Chambers 2013). Such impacts produce a large quantity of debris material that escapes the planets entirely, forming a dust disk that causes significant mid-IR excess for a period (Jackson \& Wyatt 2012; Genda et al. 2015). In this scenario, the observed rapid time variations can be linked to the orbital and collisional evolution of the dust and vapor cloud that emerged from the impact event ( $\mathrm{Su}$ et al. 2019b). Submicron-sized grains produced in optically thick clumps of vapor condensates can explain the observed prominent $10 \mu \mathrm{m}$ solid-state features ( $\mathrm{Su}$ et al. 2020).

By studying EDDs, we can learn more about the immediate aftermath of major transient dust production events possibly associated to giant impacts. Such investigations thus also have the potential to improve our knowledge on the formation of terrestrial planets. However, EDDs are rare objects, currently only $11^{1}$ such disks are known (Appendix C). To understand this phenomenon in greater detail, we need a larger sample. In the present work we aim to identify additional EDDs around Sun-like stars and study their properties with special regard to infrared variability and age distribution. The discovery of currently known EDDs in the literature was mainly based on infrared data from the Infrared Astronomical Satellite (IRAS, Neugebauer et al. 1984) survey and from dedicated Spitzer programs targeting young open clusters. In our study we use photometry obtained with the Wide-Field Infrared Survey Explorer (WISE, Wright et al. 2010), that scanned the whole sky in four infrared bands, at $3.4 \mu \mathrm{m}$ (W1), $4.6 \mu \mathrm{m}$ (W2), $12 \mu \mathrm{m}$ (W3), and $22 \mu \mathrm{m}$ (W4). Thanks to its substantially better sensitivity in the latter two bands than IRAS had at 12 and $25 \mu \mathrm{m}$, WISE offers an excellent opportunity to search for warm debris disks over the entire sky, as it has already been demonstrated by several studies (e.g., Kennedy \& Wyatt 2013; Cruz-Saenz de Miera et al. 2014; Patel et al. 2014, 2017; Cotten \& Song 2016). The two shorter wavelength

\footnotetext{
1 This number refers only to EDDs hosted by FGK-type mainsequence stars. We note that recent years have seen the discovery of several young $\mathrm{M}$ dwarf stars with warm circumstellar disks having extremely high fractional luminosity (Flaherty et al. 2019; Silverberg et al. 2020; Zuckerman et al. 2019). These systems may have a mixed nature while some of them are probably long lived accretion disks (e.g. Silverberg et al. 2020) some may harbor debris disks (Zuckerman et al. 2019).
} 
bands enable the detection and characterization of hot dust at temperatures above $400 \mathrm{~K}$. The WISE satellite is still in operation and has been continuing the all sky surveys at 3.4 and $4.6 \mu \mathrm{m}$ providing photometry spaced by 6 months. Our study on infrared variability of EDDs is principally based on this data set.

This paper is structured as follows. Section 2 reviews our investigation to search for new EDD systems utilizing the AllWISE infrared (Cutri \& et al. 2013) and the Tycho-Gaia Astrometric Solution (TGAS, Michalik et al. 2015; Lindegren et al. 2016) astrometric catalogs. Our procedure - in which we took special care to filter out possible false disk identifications - resulted in six disks. In Section 3 we discuss the characterization of the host stars including the age estimates of the systems. The determination of the disk properties is described in Section 4, while the mid-infrared variability of the selected objects is studied in Section 5. We then discuss our results (Section 6) and provide a summary of the work (Section 7).

\section{IDENTIFICATION OF NEW EDDS}

Aimed at revealing new EDDs hosted by Sun-like (F5K7-type) stars, we took mid-infrared photometric data from the AllWISE catalog and combined them with astrometric information from the TGAS database. The AllWISE data release is based on observations from the WISE cryogenic and NEOWISE post-cryogenic survey phases of the WISE mission obtained in 2010-2011. The catalog contains positions and four band (W1-W4) midIR photometric information for $\sim 750$ million sources. To reduce the chance of source confusion and contamination from strong diffuse background emission, we discarded all sources located within $5^{\circ}$ of the galactic plane. Correspondingly to our targeted spectral type range, we selected objects with $J-K_{s}$ color index between 0.22 and 0.8. The conversion between spectral types and colors was adopted from Pecaut \& Mamajek (2013). To discard suspicious WISE photometric data from the compiled sample, we applied a number of additional selection criteria. We only used sources where (1) the W1, W2, and W3 band flux measurements have signal-to-noise ratios $(\mathrm{S} / \mathrm{Ns})>10 ;(2)$ the $\mathrm{W} 4$ band measurement has an $\mathrm{S} / \mathrm{N}$ of $>3$; and (3) the reduced $\chi^{2}$ (w1rchi2-w4rchi2) of the profile-fit photometry measurement is $<3$ in each band. Moreover, we removed all objects with contamination and confusion flags (cc_flags) of either 'D', 'P', 'H' or 'O' in any of the four bands, i.e., where the photometry is probably affected by diffraction, persistence, halo, or ghost artifacts. In the finally selected sample, we corrected the potential overestimate of the real flux in saturated W2 photometry at $4.6 \mu \mathrm{m}$ by applying the formula proposed by Cotten \& Song (2016, eq. 5).

The TGAS catalog provides a 5D astrometric solution for over 2 million sources. From this data set we retained only those objects that are located within $300 \mathrm{pc}$ of the Sun, and have a good quality parallax measurement with $\pi / \sigma_{\pi}>5$. We also eliminated giant stars from the list by removing TGAS sources with $J$ band absolute magnitude $M_{J}<5 \times\left(J-K_{s}\right)$. We took near-IR photometry from the Two Micron All-Sky Survey (2MASS, Skrutskie et al. 2006). The applied criteria were developed based on Rhee et al. (2007), who used $B$ and $V$ band data, but - due to possible reddening - we based our formula on near-infrared photometry, which is less sensitive to extinction.

Accounting for the epoch difference between the TGAS (J2015.0) and AllWISE (J2010.0) catalogs, we corrected the positions of the selected TGAS objects for proper motion. After applying all of the above selection criteria, we cross-matched the two samples adopting a match radius of $00^{\prime \prime} 3$. This resulted in a combined data set including 78650 objects.

To identify new EDDs, we looked for objects exhibiting significant excess emission in the W2 and W3 bands. The W2 band excess was requested because we aim at examining the mid-IR variability of the revealed disks. To assess possible excesses in both bands, we compared the measured $K_{\mathrm{s}}-W 2$ and $K_{\mathrm{s}}-W 3$ color indices with the predicted ones. The predicted $K_{\mathrm{s}}-W 2$ and $K_{\mathrm{s}}-W 3$ $\left(P_{\mathrm{K}_{\mathrm{s}}-\mathrm{W}_{\mathrm{i}}}\right.$, where $i$ is 2 or 3$)$ values were inferred from the $J-K_{\mathrm{s}}$ color of the stars using color-color sequences quoted in table 5 of Pecaut \& Mamajek (2013). The uncertainty of this prediction $\left(\sigma_{\mathrm{P}_{\mathrm{K}_{\mathrm{s}}}-\mathrm{W}_{\mathrm{i}}}\right)$ is estimated to be $2 \%$. The significance of the excesses was then computed as

$$
\chi_{\mathrm{E}\left(\mathrm{K}_{\mathrm{s}}-\mathrm{W}_{\mathrm{i}}\right)}=\frac{E\left(K_{s}-W_{i}\right)}{\left.\sigma_{\mathrm{E}\left(\mathrm{K}_{\mathrm{s}}-\mathrm{W}_{\mathrm{i}}\right)}\right)}=\frac{K_{s}-W_{i}-P_{\mathrm{K}_{\mathrm{s}}-\mathrm{W}_{\mathrm{i}}}}{\sigma_{\mathrm{E}\left(\mathrm{K}_{\mathrm{s}}-\mathrm{W}_{\mathrm{i}}\right)}},
$$

where $\sigma_{\mathrm{E}\left(\mathrm{K}_{\mathrm{s}}-\mathrm{W}_{\mathrm{i}}\right)}$ is the quadratic sum of the measured uncertainties in the $K_{\mathrm{s}}$ and $W_{\mathrm{i}}$ bands, and $\sigma_{\mathrm{P}_{\mathrm{K}_{\mathrm{s}}-\mathrm{W}_{\mathrm{i}}}}$, i.e.: $\sigma_{\mathrm{E}\left(\mathrm{K}_{\mathrm{s}}-\mathrm{W}_{\mathrm{i}}\right)}=\sqrt{\sigma_{\mathrm{K}_{\mathrm{s}}}^{2}+\sigma_{\mathrm{W}_{\mathrm{i}}}^{2}+\sigma_{\mathrm{P}_{\mathrm{K}_{\mathrm{s}}-\mathrm{W}_{\mathrm{i}}}}^{2}}$. We selected those objects as EDD candidates where both $\chi_{\mathrm{E}\left(\mathrm{K}_{\mathrm{s}}-\mathrm{W}_{2}\right)}$ and $\chi_{\mathrm{E}\left(\mathrm{K}_{\mathrm{s}}-\mathrm{W}_{3}\right)}$ were higher than 5. This left us with 27 objects.

Looking at the candidates, we found that two of them were previously known EDDs (HD 23514 and $\mathrm{BD}+20$ 307). For a further ten stars, the spectral type found in the literature does not correspond to the interval we selected. Four among them have A0-F2 spectral type, while the other six objects are nearby M-type stars. By comparing with the proper atmosphere models, the latter six no longer show significant excess. For 
Table 1. Stellar and disk properties

\begin{tabular}{|c|c|c|c|c|c|c|c|}
\hline Parameters & TYC 4515 & TYC 5940 & TYC 8105 & TYC 4946 & TYC 4209 & TYC 4479 & References \\
\hline \multicolumn{8}{|c|}{ Identifiers } \\
\hline ТҮСНО & TYC $4515-485-1$ & TYC 5940-1510-1 & TYC $8105-370-1$ & TYC 4946-1106-1 & TYC 4209-1322-1 & TYC $4479-3-1$ & Høg et al. (2000) \\
\hline Gaia DR2 & 552973538962350080 & 2942418533272205312 & 5554553591848668672 & 3596395748683517440 & 2161325369818713984 & 2210856513228282496 & Gaia DR2 \\
\hline Gaia EDR3 & 552973538964681088 & 2942418533272205312 & 5554553591848668672 & 3596395748683517440 & 2161325369818713984 & 2210856513228282496 & Gaia EDR3 \\
\hline \multicolumn{8}{|c|}{ Astrometric Information } \\
\hline$\alpha$ R.A. (2000) & $05: 04: 07.2$ & $06: 05: 13.6$ & $06: 11: 03.5$ & $12: 13: 34.2$ & $18: 17: 03.9$ & $23: 52: 50.6$ & SIMBAD \\
\hline$\delta$ Decl. $(2000)$ & $+77: 58: 57.5$ & $-19: 13: 08.3$ & $-47: 11: 29.4$ & $-05: 35: 43.4$ & $+64: 33: 55.0$ & $+67: 30: 37.6$ & SIMBAD \\
\hline$\mu_{\alpha^{*}}\left(\operatorname{mas} \mathrm{yr}^{-1}\right)$ & $+6.710 \pm 0.012$ & $+0.011 \pm 0.010$ & $+6.277 \pm 0.012$ & $-27.754 \pm 0.023$ & $+12.051 \pm 0.014$ & $+38.619 \pm 0.016$ & Gaia EDR3 \\
\hline$\mu_{\delta}\left(\operatorname{mas~yr} r^{-1}\right)$ & $-23.625 \pm 0.015$ & $-11.719 \pm 0.012$ & $+12.577 \pm 0.014$ & $-4.047 \pm 0.018$ & $-3.106 \pm 0.013$ & $-39.644 \pm 0.014$ & Gaia EDR3 \\
\hline$\pi(\mathrm{mas})$ & $+3.564 \pm 0.015$ & $+3.784 \pm 0.013$ & $+5.411 \pm 0.010$ & $+4.093 \pm 0.021$ & $+3.647 \pm 0.010$ & $+6.142 \pm 0.014$ & Gaia EDR3 \\
\hline$R U W E$ & 0.872 & 0.964 & 0.796 & 1.214 & 0.744 & 0.888 & Gaia EDR3 \\
\hline \multicolumn{8}{|c|}{ Photometric Data } \\
\hline$B_{\mathrm{T}}$ & $11.315 \pm 0.054$ & $12.949 \pm 0.258$ & $12.486 \pm 0.191$ & $11.199 \pm 0.064$ & $12.281 \pm 0.160$ & $11.853 \pm 0.078$ & Høg et al. (2000) \\
\hline$B$ & $11.154 \pm 0.100$ & $12.694 \pm 0.021$ & $12.448 \pm 0.031$ & $11.143 \pm 0.011$ & $11.980 \pm 0.133$ & $11.769 \pm 0.075$ & Henden et al. (2016) \\
\hline$B P_{\text {Gaia }}$ & $10.8965 \pm 0.0028$ & $12.1920 \pm 0.0035$ & $11.9305 \pm 0.0038$ & $10.8298 \pm 0.0028$ & $11.7268 \pm 0.0031$ & $11.3735 \pm 0.0030$ & Gaia EDR3 \\
\hline$v_{\mathrm{T}}$ & $10.807 \pm 0.051$ & $11.923 \pm 0.156$ & $11.746 \pm 0.136$ & $10.921 \pm 0.073$ & $11.684 \pm 0.131$ & $11.153 \pm 0.069$ & Høg et al. (2000) \\
\hline v & $10.705 \pm 0.087$ & $12.007 \pm 0.070$ & $11.696 \pm 0.026$ & $10.669 \pm 0.013$ & $11.560 \pm 0.049$ & $11.156 \pm 0.048$ & Henden et al. (2016) \\
\hline$G_{\text {Gaia }}$ & $10.6430 \pm 0.0028$ & $11.8630 \pm 0.0028$ & $11.5505 \pm 0.0028$ & $10.5926 \pm 0.0028$ & $11.4509 \pm 0.0028$ & $11.0459 \pm 0.0028$ & Gaia EDR3 \\
\hline$R P_{\text {Gaia }}$ & $10.2366 \pm 0.0038$ & $11.3693 \pm 0.0040$ & $11.0042 \pm 0.0042$ & $10.2026 \pm 0.0038$ & $11.0122 \pm 0.0039$ & $10.5491 \pm 0.0038$ & Gaia EDR3 \\
\hline$J$ & $9.815 \pm 0.022$ & $10.787 \pm 0.024$ & $10.426 \pm 0.024$ & $9.793 \pm 0.023$ & $10.556 \pm 0.023$ & $9.972 \pm 0.023$ & Cutri et al. (2003) \\
\hline$H$ & $9.620 \pm 0.021$ & $10.518 \pm 0.022$ & $10.028 \pm 0.022$ & $9.586 \pm 0.022$ & $10.267 \pm 0.023$ & $9.663 \pm 0.031$ & Cutri et al. (2003) \\
\hline$K_{\mathrm{s}}$ & $9.538 \pm 0.019$ & $10.402 \pm 0.019$ & $9.934 \pm 0.021$ & $9.520 \pm 0.023$ & $10.139 \pm 0.016$ & $9.624 \pm 0.018$ & Cutri et al. (2003) \\
\hline \multicolumn{8}{|c|}{ Kinematics } \\
\hline$v_{\mathrm{r}, \text { our }}\left(\mathrm{km} \mathrm{s}^{-1}\right)$ & $+12.50 \pm 0.50$ & $+28.50 \pm 0.20$ & $+29.00 \pm 0.20$ & $-11.30 \pm 0.50$ & $-13.20 \pm 0.60$ & $+0.22 \pm 0.30$ & This work \\
\hline$v_{\mathrm{r}, \mathrm{Gaia}}\left(\mathrm{km} \mathrm{s}^{-1}\right)$ & $-2.65 \pm 0.89$ & $+30.52 \pm 1.38$ & $+29.06 \pm 0.65$ & $-12.29 \pm 0.36$ & $-13.46 \pm 1.14$ & $\cdots$ & Gaia DR2 \\
\hline$U_{\left(\mathrm{km} \mathrm{s}^{-1}\right)}^{b}$ & $-32.7 \pm 0.3$ & $-8.1 \pm 0.1$ & $-16.9 \pm 0.1$ & $-27.2 \pm 0.2$ & $+3.0 \pm 0.1$ & $-21.2 \pm 0.1$ & This work \\
\hline$V^{b}\left(\mathrm{~km} \mathrm{~s}^{-1}\right)$ & $-11.3 \pm 0.3$ & $-27.4 \pm 0.1$ & $-25.9 \pm 0.2$ & $-12.3 \pm 0.3$ & $-4.1 \pm 0.5$ & $-7.0 \pm 0$ & This work \\
\hline${ }_{W} b_{\left(\mathrm{km} \mathrm{s}^{-1}\right)}$ & $-3.7 \pm 0.2$ & $-14.5 \pm 0.1$ & $-5.8 \pm 0.1$ & $-16.5 \pm 0.4$ & $-20.1 \pm 0.3$ & $-36.2 \pm 0.1$ & This work \\
\hline Distance $(\mathrm{pc}) C$ & $278.8 \pm 1.1$ & $262.1_{-1.0}^{+1.2}$ & $184.1_{-0.3}^{+0.4}$ & $242.1_{-1.1}^{+1.4}$ & $270.8_{-0.6}^{+0.7}$ & $162.0_{-0.3}^{+0.4}$ & Bailer-Jones et al. (2020) \\
\hline \multicolumn{8}{|c|}{ Stellar properties } \\
\hline Spectral Type & F5V & G5V & G9V & F6V & G1V & G6V & This work \\
\hline$T_{\text {eff }}(\mathrm{K})$ & $6540 \pm 100$ & $5660 \pm 100$ & $5350 \pm 100$ & $6350 \pm 60$ & $5890 \pm 120$ & $5620 \pm 100$ & This work \\
\hline $\log g(\mathrm{cgs})$ & $4.28 \pm 0.10$ & $4.49 \pm 0.10$ & $4.48 \pm 0.10$ & $4.13 \pm 0.06$ & $4.40 \pm 0.10$ & $4.30 \pm 0.10$ & This work \\
\hline$[\mathrm{Fe} / \mathrm{H}](\mathrm{dex})$ & $+0.07 \pm 0.10$ & $+0.06 \pm 0.10$ & $+0.08 \pm 0.10$ & $-0.02 \pm 0.05$ & $+0.01 \pm 0.12$ & $-0.29 \pm 0.10$ & This work \\
\hline$L_{*}\left(\mathrm{~L}_{\odot}\right)$ & $3.72 \pm 0.12$ & $0.94 \pm 0.03$ & $0.65 \pm 0.02$ & $2.55 \pm 0.08$ & $1.43 \pm 0.04$ & $0.76 \pm 0.02$ & This work \\
\hline$M_{*}\left(\mathrm{M}_{\odot}\right)$ & $1.32 \pm 0.04$ & $0.99_{-0.03}^{+0.04}$ & $0.94 \pm 0.03$ & $1.17_{-0.03}^{+0.04}$ & $1.05 \pm 0.03$ & $0.84 \pm 0.03$ & This work \\
\hline$E W_{\mathrm{Li}}(\mathrm{m} \AA)$ & $<15.0$ & $157 \pm 8$ & $173 \pm 6$ & $38 \pm 8$ & $101 \pm 15$ & $13 \pm 4$ & This work \\
\hline$P_{\text {rot }}($ day $)$ & r & 3.76 & 5.04 & $\ldots$ & 5.07 & 32.1 & This work \\
\hline Age (Myr) & $>150$ & $120 \pm 20$ & $130 \pm 30$ & $>150$ & $275 \pm 50$ & $5000 \pm 2000$ & This work \\
\hline Multiplicity & $\mathrm{Y}$ & $\mathrm{N}$ & $\mathrm{Y}$ & $\mathrm{Y}$ & Y & $\mathrm{Y}$ & This work \\
\hline \multicolumn{8}{|c|}{ Disk properties } \\
\hline $\mathrm{T}_{\text {disk, bb }}(\mathrm{K})$ & $500 \pm 25$ & $420 \pm 16$ & $300 \pm 20$ & $680 \pm 45$ & $530 \pm 15$ & $400 \pm 25$ & This work \\
\hline $\mathrm{f}_{\mathrm{d}}$ & 0.015 & 0.037 & 0.052 & 0.010 & 0.070 & 0.014 & This work \\
\hline $\mathrm{R}_{\text {disk,bb }}(\mathrm{au})$ & 0.60 & 0.43 & 0.69 & 0.27 & 0.33 & 0.42 & This work \\
\hline $\mathrm{f}_{\mathrm{d}} / \mathrm{f}_{\max }\left(10^{5}\right)$ & 1.1 & 1.9 & 0.8 & 3.4 & 20.0 & 25.4 & This work \\
\hline mid-IR variations & $\mathrm{N}$ & $\mathrm{Y}$ & $\mathrm{Y}$ & $\mathrm{N}$ & $\mathrm{Y}$ & $\mathrm{Y}$ & This work \\
\hline
\end{tabular}

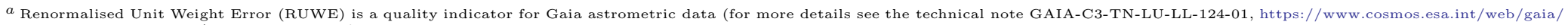
public-dpac-documents). RUWE values of $<1.4$ indicate reliable astrometric solutions.

${ }^{b}$ In computation of the $U, V, W$ velocity components, we used the radial velocity data obtained by us $\left(v_{\mathrm{r}}\right.$,our $)$.

$c$ Geometric distances from Bailer-Jones et al. (2020).

${ }^{d}$ Lithium equivalent widths after correction for blending with the iron line (Sect. 3.4). 
ten objects the spectral energy distribution based on W1-W4 data is parallel to the stellar photosphere defined by the 2MASS measurements, but at a higher absolute level. Based on the literature all of these objects are eclipsing binaries. According to the AllWISE catalog, six of them were probably variable in the W2 band as indicated by the value of their variability flag (var_flag) that has been set to the highest possible value of 9 . Four of these objects probably displayed substantial changes in the $\mathrm{W} 3$ band, too. For these ten binaries, our predicted photospheric colors $\left(P_{\mathrm{K}_{\mathrm{s}}-\mathrm{W}_{2}}\right.$, $\left.P_{\mathrm{K}_{\mathrm{s}}-\mathrm{W}_{3}}\right)$ are probably inaccurate, and our excess detection is false. We note that two of these objects were already removed from our list because they have a spectral type that is too early. The WISE photometry of CD-48 8486, a G-type member of the Upper Centaurus Lupus group (Mamajek et al. 2002), also indicates an SED shifted upward with respect to the 2MASS nearinfrared data, suggesting that the excess identification is dubious. Earlier mid-infrared photometric and spectroscopic observations of this source with the Spitzer confirm this suspicion: the object shows no significant excess at wavelengths $<25 \mu \mathrm{m}$ (Silverstone et al. 2006; Carpenter et al. 2009).

The literature lists 11 EDDs with F-K-type host stars (Appendix C). Out of this sample we recovered two objects, HD 23514 and BD+20 307. The other nine objects were excluded by our searching algorithm because of various reasons. HD 15407 and HD 166191 lie too close to the Galactic plane $\left(|b|<5^{\circ}\right)$, while RZ Psc, V488 Per, HD 113766, ID 8 and P 1121 are not included in the TGAS catalog. HD 145263 has an earlier spectral type than the other targets, its $J-K_{s}$ color index is outside our specified range, while the disk of TYC 824126521 had been substantially depleted before the start of WISE mission (Appendix C) and no longer exhibited strong excess in the $\mathrm{W} 2$ and $\mathrm{W} 3$ bands.

The detailed analysis, described above, left us with 6 EDD candidates out of the 27 initially selected sources. They are: TYC 4515-485-1 (hereafter TYC 4515), TYC 5940-1510-1 (TYC 5940), TYC 8105370-1 (TYC 8105), TYC 4946-1106-1 (TYC 4946), TYC 4209-1322-1 (TYC 4209), and TYC 4479-3-1 (TYC 4479). However, due to the large WISE beam source, confusion or contamination by extended emission cannot be ruled out even in these cases. In order to test this, we performed several additional checks, but based on the currently available infrared data we found no sign of confusion or significant contamination at any of the selected systems (Appendix A). Therefore, throughout the following analysis, we will assume that the excesses in all six cases come from warm cir- cumstellar dust disks. Previously, one of these objects, TYC 4479, was identified as a debris disk system (Cotten \& Song 2016), while the other five disks are new discoveries.

Figure 1 shows the vicinity of the six systems in all four WISE bands (using the "unWISE" coadds, see Appendix A), while Table 1 summarizes astrometric and photometric data of the host stars. Although, during the selection process, we used TGAS astrometry, in this table we list more precise astrometric parameters taken from the Gaia EDR3 catalog (Gaia Collaboration et al. 2020; Lindegren et al. 2020).

\section{CHARACTERIZATION OF THE HOST STARS}

\subsection{Spectroscopic observations}

In order to estimate the fundamental parameters of the host stars of the identified EDDs, we performed high-resolution optical spectroscopy for all of them. As the observation log (Table 2) shows we used four different high-resolution échelle spectrographs to obtain the spectra. One of our targets, TYC 4946, was measured twice.

TYC 5940, TYC 8105, and TYC 4946 were targeted with the Fibre-fed Extended Range Optical Spectrograph (FEROS, Kaufer et al. 1999) on the MPG/ESO $2.2 \mathrm{~m}$ (La Silla, Chile) telescope. This instrument has a mean spectral resolution of 48000 , covering the spectral region between 3500 and $9200 \AA$. In our observations, the object-sky operation mode was used, i.e., one of the two fibres was positioned at the star while the other at the sky. Reduction of FEROS spectra - including bias subtraction, flat-field correction, background subtraction, the definition and extraction of orders, and wavelength calibration - were performed using the FEROS Data Reduction System $\left(\mathrm{DRS}^{2}\right)$ pipeline implemented in the ESO-MIDAS environment.

We obtained spectra of TYC 4515 and TYC 4946 at the Apache Point Observatory (New Mexico, US), using the ARC Echelle Spectrograph (ARCES) on the $3.5 \mathrm{~m}$ telescope with a resolution of $R=31500$ in the spectral range $3200-10000 \AA$. The spectra were reduced using

\footnotetext{
2 http://www.eso.org/sci/facilities/lasilla/instruments/feros/ tools/DRS.html
} 


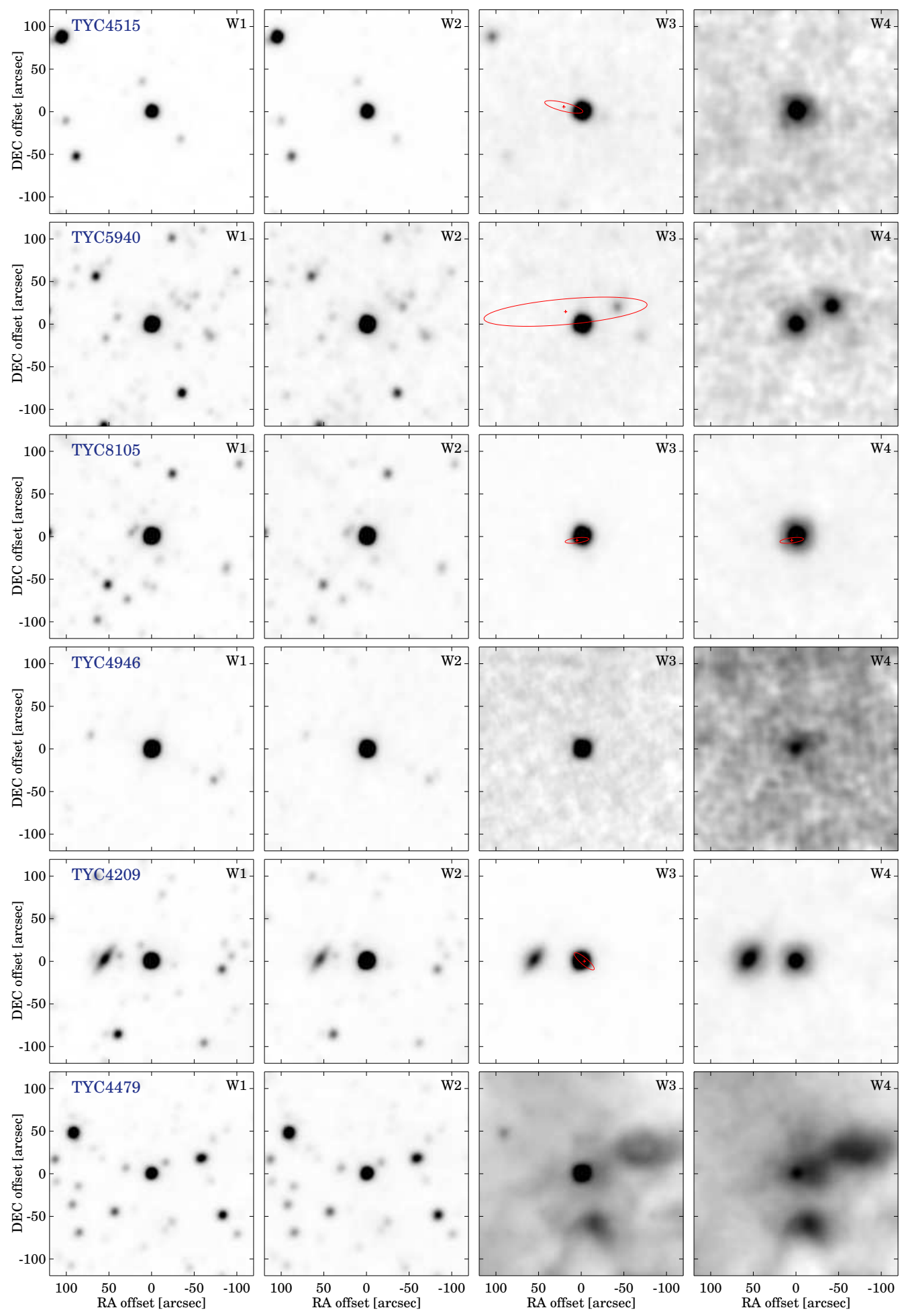

Figure 1. W1-W4 band images (unWISE coadds of the WISE all sky survey, see Appendix A) of the six selected objects.

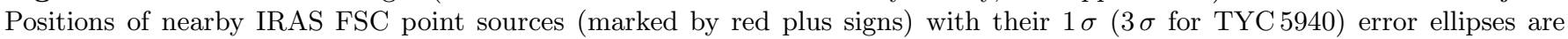
overplotted in the W3 band panels. 
a dedicated pipeline ${ }^{3}$ based on $\operatorname{IRAF}^{4}$, then normalized to the continuum level.

TYC 4479 was observed using The FIbre-fed Échelle Spectrograph (FIES, Frandsen \& Lindberg 1999; Telting et al. 2014) on the $2.56 \mathrm{~m}$ Nordic Optical Telescope (Observatorio del Roque de los Muchachos, La Palma, Spain) in its low-res mode $(R=25000$, with wavelength coverage from 3700 to $7300 \AA$ ). Data processing was made with FIEStool $^{5}$, the dedicated pipeline of the FIES instrument.

Table 2. Log of spectroscopic observations

\begin{tabular}{c|ccc}
\hline \hline \multicolumn{1}{c}{ Name } & Instrument & Obs. date & Exposure time $(\mathrm{s})$ \\
\hline TYC 4515 & ARCES & $2013-03-25$ & 780 \\
TYC 5940 & FEROS & $2016-12-17$ & 900 \\
TYC 8105 & FEROS & $2016-12-17$ & 900 \\
TYC 4946 & FEROS & $2012-12-25$ & 1022 \\
& ARCES & $2013-04-01$ & 840 \\
TYC 4209 & ACE & $2016-09-29$ & $3 \times 5400+1 \times 3600$ \\
TYC 4479 & FIES & $2020-07-03$ & 2760 \\
\hline
\end{tabular}

Finally, the spectrum of TYC 4209 was taken with the ACE spectrograph mounted on the $1 \mathrm{~m}$ RCC telescope at Piszkéstető Observatory (Hungary). The fibre-fed

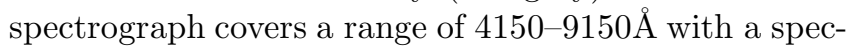
tral resolution of $\approx 20000$. For the wavelength calibration, a thorium-argon (ThAr) lamp was used. Data reduction was performed using standard IRAF procedures in the imred and echelle packages. Cosmic ray correction was done by the crutil.cosmicrays utility. For the analysis, we combined 4 frames (one with a 3600 s exposure time and three with a $5400 \mathrm{~s}$ exposure time).

\subsection{Basic stellar properties}

The preparation of the reduced spectra consisted of identification and removal of non-spectral features remaining from the reduction processes, and a correction of the continuum normalization. These steps were carried out utilizing IRAF tasks and the iSpec tool of Blanco-Cuaresma et al. (2014). Before fitting the stellar parameters, the radial velocity had to be determined

${ }^{3}$ http://astronomy.nmsu.edu:8000/apo-wiki/attachment/wiki/ ARCES/Thorburn_ARCES_manual.pdf and http://astronomy. nmsu.edu:8000/apo-wiki/attachment/wiki/ARCES/Kinemuchi_ ARCES_cookbook.pdf

${ }^{4}$ IRAF is distributed by the National Optical Astronomy Observatories, which are operated by the Association of Universities for Research in Astronomy, Inc., under cooperative agreement with the National Science Foundation.

${ }^{5}$ http://www.not.iac.es/instruments/fies/fiestool/FIEStool.html and the necessary correction had to be applied. These tasks were also performed with the iSpec tool.

For the determination of stellar parameters $\left(\log g, T_{\text {eff }}\right.$, and $[\mathrm{Fe} / \mathrm{H}])$ of the target stars we used the SP_Ace program (v1.1) written and developed by Boeche \& Grebel (2016). SP_Ace was designed for analyzing spectra with resolution between $\sim 1000$ and $\sim 20000$, but higher resolutions may be doable as well. In this range, the line profiles are plausible, since the effects of the applied approximations have been tested to not emerge. The SP_Ace methodology offers a fast and accurate solution of spectra in this range.

SP_Ace is an equivalent width (EW) based tool (the EW library was computed by MOOG, Sneden 1973), which performs the spectral analysis under LTE assumption and based on 1D atmosphere models. It uses the General Curve-Of-Growth library (GCOG) to compute the EWs of the lines, construct models of spectra as a function of the stellar parameters and abundances, and search for a model that minimizes the $\chi^{2}$ when compared to the observed spectrum. In SP_Ace, we covered the stellar parameter ranges of $3600 \mathrm{~K}<T_{\text {eff }}<7400 \mathrm{~K}$, $0.2<\log g<5.4$ and $-2.4 \operatorname{dex}<[\mathrm{Fe} / \mathrm{H}]<0.4$ dex.

To use this code, the spectra must be wavelength calibrated, continuum normalized, and radial velocity corrected at rest frame. They must be in the wavelength ranges $5212-6860 \AA$ and $8400-8924 \AA$ and must have less than 32000 pixels. Because of the last requirement, when necessary we downgraded the spectral resolution. To test how the precision behaves after such downgrading, we analyzed publicly available spectra of stars with well known spectral parameters and found that there are no significant differences between the parameters derived from the spectra with different spectral resolution in the $\sim 20000-40000$ resolution range.

The estimated stellar parameters and their uncertainties are summarized in Table 1. As a final step, the error of the radial velocity is revised with the resulting stellar parameters. The obtained radial velocities are also listed in Table 1.

In order to predict the stellar photospheric contribution to the total flux at mid-IR wavelengths, we fitted the optical and near-infrared photometry of the sources (taken from Table 1) by the appropriate ATLAS9 stellar atmosphere model (Castelli \& Kurucz 2004) selected based on the previously derived stellar parameters. In the fitting process, two parameters, the amplitude of the photospheric model and the interstellar reddening (adopting the extinction curve from Cardelli et al. 1989) were estimated using a grid-based approach. Initially we used all 2MASS (Skrutskie et al. 2006) photometric data but we found that the longest wavelength $K_{\mathrm{s}}$ 
data points were systematically higher than the fitted photosphere model, suggesting that the measured fluxes are not purely photospheric. Therefore, we discarded the $K_{\mathrm{s}}$ band photometry from this analysis. Although our targets are located farther away than 160 pc, i.e., likely out of the Local Bubble, apart from the case of TYC 4515 - where an E(B-V) value of $0.04 \pm 0.02 \mathrm{mag}$ was obtained - their reddening values were found to be negligible based on this fitting. Combining these models with the Gaia EDR3 distances, we derived the stellar luminosities, also listed in Table 1.

\subsection{Stellar companions}

To explore possible stellar companions, we used the Gaia EDR3 catalog to search for common proper motion and distance pairs of our targets. Following the considerations from Andrews et al. (2017), we set a limit of $1 \mathrm{pc}$ for the maximum separation of the possible pairs. To ensure reliable quality astrometric solutions, we considered only stars whose parallax and proper motion measurements fulfilled the following criteria: $\pi / \sigma_{\pi}>5$ and $\frac{\sqrt{\mu_{\alpha *}^{2}+\mu_{\delta}^{2}}}{\sqrt{\sigma_{\mu_{\alpha *}}^{2}+\sigma_{\mu_{\delta}}^{2}}}>5$, respectively. We identified companions by applying the method described in Deacon \& Kraus (2020), which takes into account the possible orbital motion of the components. We found probable wide separation companions to TYC 4515, TYC 8105, TYC 4946, TYC 4209, and TYC 4479, i.e., to all stars with the exception of TYC 5940. All of the companion stars have $R U W E^{6}<1.4$, indicating a well-fitting astrometric solution. Despite the large search radius, the projected separations of the identified pairs range between $1000(\sim 0.005 \mathrm{pc})$ and $6000 \mathrm{au}(\sim 0.03 \mathrm{pc})$. This means that both components can be formed by the fragmentation of a single protostellar core, that has a typical size of 0.1 pc (Lada et al. 2008; Duchêne \& Kraus 2013). Table 3 shows the astrometric parameters for the five pairs.

For the companions of TYC 4515, TYC 8105, TYC 4209, and TYC 4479, we estimated the effective temperatures based on their $r-z, r-J$, and $G_{B P}-$ $G_{R P}$ color indices, using the calibration derived by Mann et al. (2015). The $G_{B P}$ and $G_{R P}$ band data were collected from the GaiaEDR3 catalog. The $J$ band measurements were extracted from the 2MASS survey (Skrutskie et al. 2006). For TYC 8105B, the $r$ and $z$ band photometry were taken from The Dark Energy

${ }^{6}$ Renormalised Unit Weight Error (RUWE) is an indicator of the goodness of fit for Gaia astrometric data: values $<1.4$ are considered reliable (for more details, see the technical note GAIA-C3-TN-LU-LL-124-01, https://www.cosmos.esa.int/web/ gaia/public-dpac-documents).
Survey (DR1, Abbott et al. 2018), while in the cases of TYC 4515B and TYC 4209B, magnitudes measured in The Pan-STARRS Survey (DR1, Chambers et al. 2016) were transformed into the SDSS system using the equations presented in Tonry et al. (2012). For TYC 4209B, that was observed both in The SDSS Photometric Catalog (Release 12, Alam et al. 2015) and The Pan-STARRS Survey, we derived the final $r$ and $z$ photometry as the weighted average of these data (after the transformation of the Pan-STARRS observations to the SDSS photometric system). Photometry for TYC 4515B were dereddened by adopting the $E(B-V)$ values obtained for their primary components (Sect. 3.2). The final $T_{\text {eff }}$ values, which are the weighted averages of the three $T_{\text {eff }}$ estimates corresponding to the three colors, are listed in Table 3.

The spectral types of the companions were estimated based on the obtained effective temperatures using an updated version ${ }^{7}$ of the conversion from Pecaut \& Mamajek (2013). We found that the spectral types of these secondary components range between M1 and M5.5, thus, these are newly discovered M dwarfs. We also derived the stellar luminosities. As a first step, we performed bolometric correction to the $G, r, z, J$, $H$, and $K_{\mathrm{s}}$ passbands using the proper equations of Mann et al. (2015). The final bolometric magnitudes were obtained as the weighted average of the six individual bolometric magnitudes. Then, considering the reddening and adopting the distances of the primary components (which have substantially lower uncertainties than those of the companions) we calculated the luminosities of the four companions (Table 3).

For TYC 4946B, where reliable photometry was available only in the $G$ band, the $T_{\text {eff }}, L_{*}, M_{*}$ parameters and the spectral type were estimated based on its $G$ band absolute magnitude by interpolating in the updated table of Pecaut \& Mamajek (2013). The obtained parameters of this $\sim$ M4.5-type star are also listed in Table 3 .

We also investigated the possible existence of closer binary pairs in our sample. Qian et al. (2019) listed TYC 4946 as a possible spectroscopic binary, because its two observations in the LAMOST (The Large Sky AreaMulti-Object Fiber Spectroscopic Telescope) survey brought very different radial velocity measurements $\left(v_{\mathrm{r}}=-37.99 \pm 0.72 \mathrm{~km} \mathrm{~s}^{-1}\right.$ and $-16.30 \pm$ $\left.3.43 \mathrm{~km} \mathrm{~s}^{-1}\right)$. For this star, we obtained radial velocities of $-10.5 \pm 0.7 \mathrm{~km} \mathrm{~s}^{-1}$ and $-12.1 \pm 0.7 \mathrm{~km} \mathrm{~s}^{-1}$ from the observations carried out by the ARCES and FEROS spectrographs at two different epochs (Table 2). The

\footnotetext{
7 https://www.pas.rochester.edu/ emamajek/EEM_dwarf_ UBVIJHK_colors_Teff.txt
} 
Table 3. Astrometric parameters and stellar properties of the probable common motion and distance pairs

\begin{tabular}{|c|c|c|c|c|c|c|c|c|c|c|}
\hline Name & Gaia EDR3 id & $\begin{array}{c}\text { Separation } \\
\text { (au) }\end{array}$ & $\begin{array}{c}\mu_{\alpha^{*}} \\
\left(\operatorname{mas~yr}{ }^{-1}\right)\end{array}$ & $\begin{array}{c}\mu_{\delta} \\
\left(\operatorname{mas} \mathrm{yr}^{-1}\right)\end{array}$ & $\begin{array}{c}\pi \\
\text { (mas) }\end{array}$ & $\begin{array}{c}G \\
(\mathrm{mag})\end{array}$ & $\mathrm{SpT}$ & $\begin{array}{l}T_{\text {eff }} \\
(\mathrm{K})\end{array}$ & $\begin{array}{c}L_{*} \\
\left(L_{\odot}\right)\end{array}$ & $\begin{array}{c}M_{*} \\
\left(\mathrm{M}_{\odot}\right)\end{array}$ \\
\hline TYC 4515B & 552973534667883904 & 1825 & $+6.61 \pm 0.05$ & $-24.18 \pm 0.08$ & $3.49 \pm 0.06$ & 16.578 & M1 & $3670 \pm 60$ & $0.0363 \pm 0.0014$ & $0.50 \pm 0.02$ \\
\hline TYC 8105B & 5554553527426091136 & 4540 & $+6.80 \pm 0.33$ & $+13.54 \pm 0.39$ & $5.57 \pm 0.30$ & $19.510^{a}$ & M5.5 & $2960 \pm 60$ & $0.0024 \pm 0.0001$ & $0.096 \pm 0.01$ \\
\hline TYC 4946B & 3596395748685297792 & 1010 & $-27.66 \pm 0.34$ & $-5.07 \pm 0.26$ & $4.08 \pm 0.28$ & $18.812^{a}$ & M4.5 & $\sim 3120$ & $\sim 0.005$ & $\sim 0.19$ \\
\hline TYC 4209B & 2161326842991191936 & 6010 & $+12.13 \pm 0.17$ & $-3.38 \pm 0.17$ & $3.85 \pm 0.11$ & 18.337 & M3.5 & $3230 \pm 55$ & $0.0077 \pm 0.0003$ & $0.27 \pm 0.01$ \\
\hline TYC 4479B & 2210856513228282880 & 5040 & $+38.90 \pm 0.04$ & $-39.53 \pm 0.04$ & $6.12 \pm 0.03$ & 15.867 & M2 & $3540 \pm 60$ & $0.0182 \pm 0.0006$ & $0.38 \pm 0.02$ \\
\hline
\end{tabular}

${ }^{a}$ Corrected G-band magnitude for sources with 6-parameter astrometric solutions based on Riello et al. (2020).

Gaia DR2 catalog (Gaia Collaboration et al. 2018b) in good agreement with our results - quotes a $v_{\text {r of }}$ $-12.29 \pm 0.36 \mathrm{~km} \mathrm{~s}^{-1}$. In the case of TYC 4515 , we extracted $v_{\mathrm{r}}$ of $+12.5 \pm 0.5 \mathrm{~km} \mathrm{~s}^{-1}$ from our high resolution spectrum (Table 1) which deviates significantly from its $v_{\mathrm{r}}$ of $-2.65 \pm 0.89 \mathrm{~km} \mathrm{~s}^{-1}$ measured by Gaia. These two stars are candidate spectroscopic binaries, but further measurements are needed to confirm this claim. If TYC 4515 or TYC 4946 has a spectroscopic binary pair, then they would be triple systems. For TYC 5940,

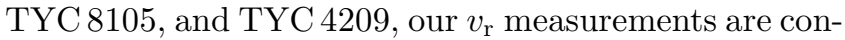
sistent with the Gaia based values, thus, there is no indication for any close companion.

\subsection{Age of the stars}

The ages of the host stars provide important information in assessing the evolutionary status of their disks. To infer this parameter, we combined a variety of age diagnostics based on lithium content, stellar rotation, and kinematic properties. In the case of TYC 8105, isochrone fitting for its M-type companion was also performed.

In stars having a convective layer, as in our objects, photospheric lithium is thought to be depleted with time when mixed into deeper layers due to convection, and destroyed at a temperature of about $2.5 \times 10^{6} \mathrm{~K}$. We measured the lithium content using the resonance doublet of $\mathrm{Li} \mathrm{I}$ at $6707.7 \AA$, whose equivalent widths $\left(\mathrm{EW}_{\mathrm{Li}}\right)$ were measured by fitting a Gaussian profile to the absorption feature in our high resolution spectra (see Sect. 3.1). The Li absorption line is blended with a weak Fe I line at $6707.4 \AA$. Taking into account this effect, the obtained equivalent widths were corrected using a method developed by Soderblom et al. (1993). For TYC 4946, where more than one spectrum were available, the final $\mathrm{EW}_{\mathrm{Li}}$ was computed as the average of the individual measurements. For TYC 4515, where the lithium line was not detected, a $3 \sigma$ upper limit was calculated. The corrected $\mathrm{EW}_{\mathrm{Li}}$ values are given in Tab. 1 and displayed as a function of effective temperatures in
Fig. 2. For comparison, members of some well-dated nearby open clusters and moving groups, representing an age range of $\sim 40-4300 \mathrm{Myr}$, are also plotted.

Late-type dwarf stars lose mass and angular momentum via magnetized stellar winds, leading to rotational spin down as they age (Schatzman 1962; Kraft 1967; Kawaler 1988). This enables using stellar rotation to estimate the ages of stars via a technique called gyrochronology (Barnes 2003). Starspots may cause periodic light changes from which the rotation period can be derived. In our sample, five stars (TYC 4515, TYC 5940, TYC 8105, TYC 4209, and TYC 4479) were observed with the TESS satellite, providing high accuracy photometry with 30 minutes cadence. All five stars exhibit periodic variations likely due to rotational modulation by star-spots. By performing a frequency analysis, we obtained rotational periods as listed in Table 1. A detailed description of the TESS data reduction and the frequency analysis is given in Appendix B. In Fig. 3 we plot the rotational periods as a function of the effective temperatures for those four G-type stars for which gyrochronology is applicable. Due to rotational evolution, stellar groups of different ages populate distinct, well-defined areas in this plot, as demonstrated for four open clusters: Pleiades ( $120 \mathrm{Myr})$, Hyades and Praesepe (both 600-800 Myr), and M67 (3.8-4.3 Gyr). Having similar ages, the members of Hyades and Praesepe overlap in this diagram.

By comparing the effective temperatures and luminosities of the six host stars (Table 1) and the four companions (Table 3) with the corresponding mean properties of dwarf stars 8 , we found that all, but TYC 8105 B, are consistent with the main-sequence. TYC $8105 \mathrm{~B}$ is brighter than main-sequence dwarfs with comparable $T_{\text {eff }}$, suggesting that it is in pre-main sequence evolution. To verify the age estimate of the TYC 8105 system, we

\footnotetext{
8 http://www.pas.rochester.edu/ emamajek/EEM_dwarf_ UBVIJHK_colors_Teff.txt (compiled by E. Mamajek)
} 


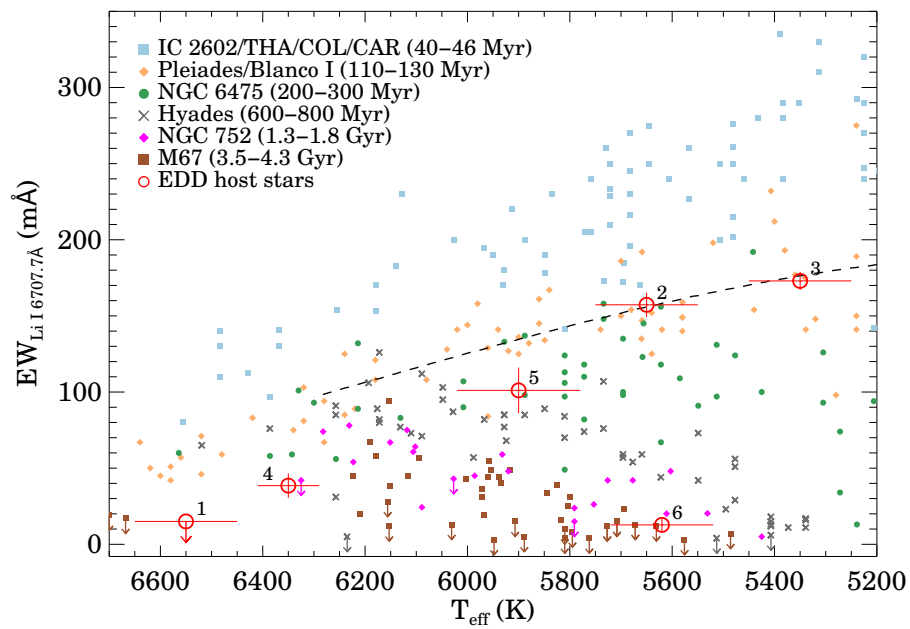

Figure 2. Equivalent width of the lithium line (at $6707.7 \AA$ ) as a function of effective temperature for our targets (1: TYC 4515, 2: TYC 5940, 3: TYC 8105, 4: TYC 4946, 5: TYC 4209, 6: TYC 4479) and for members of some nearby open clusters and moving groups with well known ages. Data for open clusters were taken from Sestito \& Randich (2005) via the WEBDA database ${ }^{\mathrm{b}}$, while for the Tucana-Horologium (THA), Columba (COL), and Carina (CAR) moving groups the data were from da Silva et al. (2009). The dashed curve stands for a polynomial fit to the lithium data of Pleiades (Pecaut \& Mamajek 2016). Ages of moving groups and open clusters were taken from the literature: IC 2602 (Dobbie et al. 2010); THA, COL, and CAR (Bell et al. 2015); Pleiades (Stauffer et al. 1998; Dahm 2015); Blanco I (Cargile et al. 2010; Juarez et al. 2014); NGC 6475 (Villanova et al. 2009; Cummings \& Kalirai 2018; Bossini et al. 2019); Hyades (Douglas et al. 2019, and references therein); NGC 752 (Daniel et al. 1994; Twarog et al. 2015; Agüeros et al. 2018); M 67 (Giampapa et al. 2006; VandenBerg \& Stetson 2004; Sarajedini et al. 2009; Bossini et al. 2019).

\footnotetext{
a https://webda.physics.muni.cz/

b https://webda.physics.muni.cz/
}

performed isochrone-fitting (see below) for the companion using isochrone tracks constructed by Baraffe et al. (2015).

Using astrometric data (coordinates, proper motions, and trigonometric parallax) from the Gaia EDR3 catalog, supplemented by our radial velocity measurements from Table 1 , we computed the $U, V$, and $W$ Galactic space velocity components of the targets. In this calculation, we used a right-handed coordinate system thus $U$ is positive toward the Galactic center, $V$ is positive in the direction of galactic rotation, and $W$ is positive toward the galactic North pole. The derived Galactic space velocities are listed in Tab. 1 and displayed in Fig. 4. For TYC 4515 and TYC 4946 where some radial velocity data in the literature differ substantially

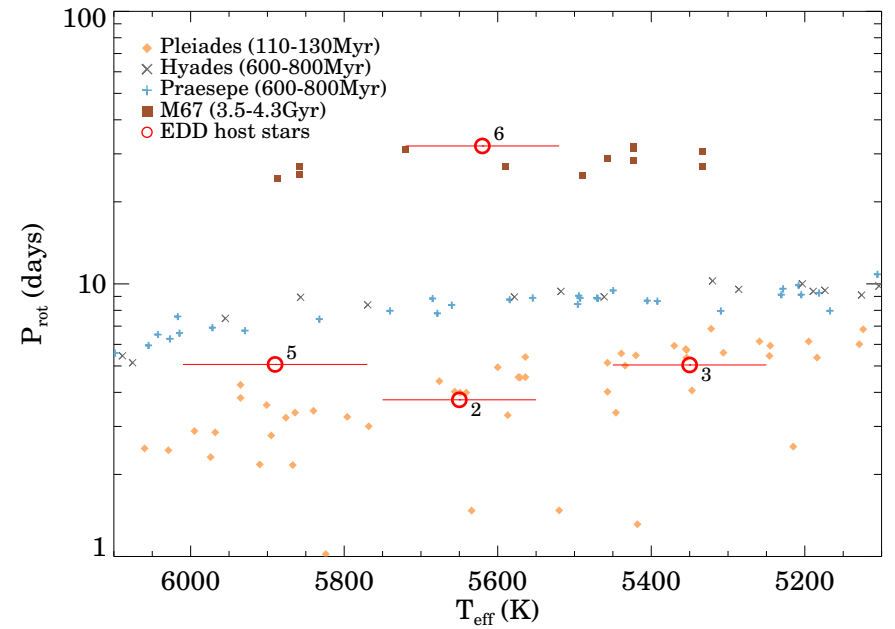

Figure 3. Rotation periods as a function of effective temperatures for our targets and for single star members of three open clusters. Our targets (TYC 5940, TYC 8105, TYC 4209, and TYC 4479) have the same labels as in Fig. 2. Data for open cluster members were from the literature (Rebull et al. 2016; Stauffer et al. 2016; Barnes et al. 2016; Douglas et al. 2019).

from our measurements (Sect. 3.3) the UVW components were computed using the most different $v_{\mathrm{r}}$ data, too and displayed in Fig. 4. For reference, known moving groups, associations, and open clusters located within $300 \mathrm{pc}$ are also plotted.

TYC5940 and TYC8105 - are both G-type stars (Tab. 1). As Fig. 2 demonstrates, their lithium equivalent widths are consistent with the Pleiades stars $(\sim 120 \mathrm{Myr})$, and lower than the values measured in the $40-50 \mathrm{Myr}$ old members of nearby moving groups and of the IC 2602 open cluster.

In line with this, the position of both stars in the period-effective temperature diagram (Fig. 3) also matches the sequence of the Pleiades. To estimate the gyrochronology age, we applied four different calibrations for the rotation-age relation (Barnes 2007; Mamajek \& Hillenbrand 2008; Meibom et al. 2009; Angus et al. 2015). For TYC 5940, these calibrations yielded age estimates between 92 and $134 \mathrm{Myr}$ with an average of $118 \mathrm{Myr}$, while the gyroages for TYC 8105 range from 125 to $197 \mathrm{Myr}$ with an average of $163 \mathrm{Myr}$.

As Fig. 4 outlines, the UVW velocities of both stars are consistent with the typical space motion of nearby young (10-200 Myr old) stars. Interestingly, the derived $U, V, W$ components of TYC 5940 overlap with the Pleiades and the AB Dor moving groups. We note, however, that the direction and distance of this star are clearly inconsistent with those of both Pleiades and the 

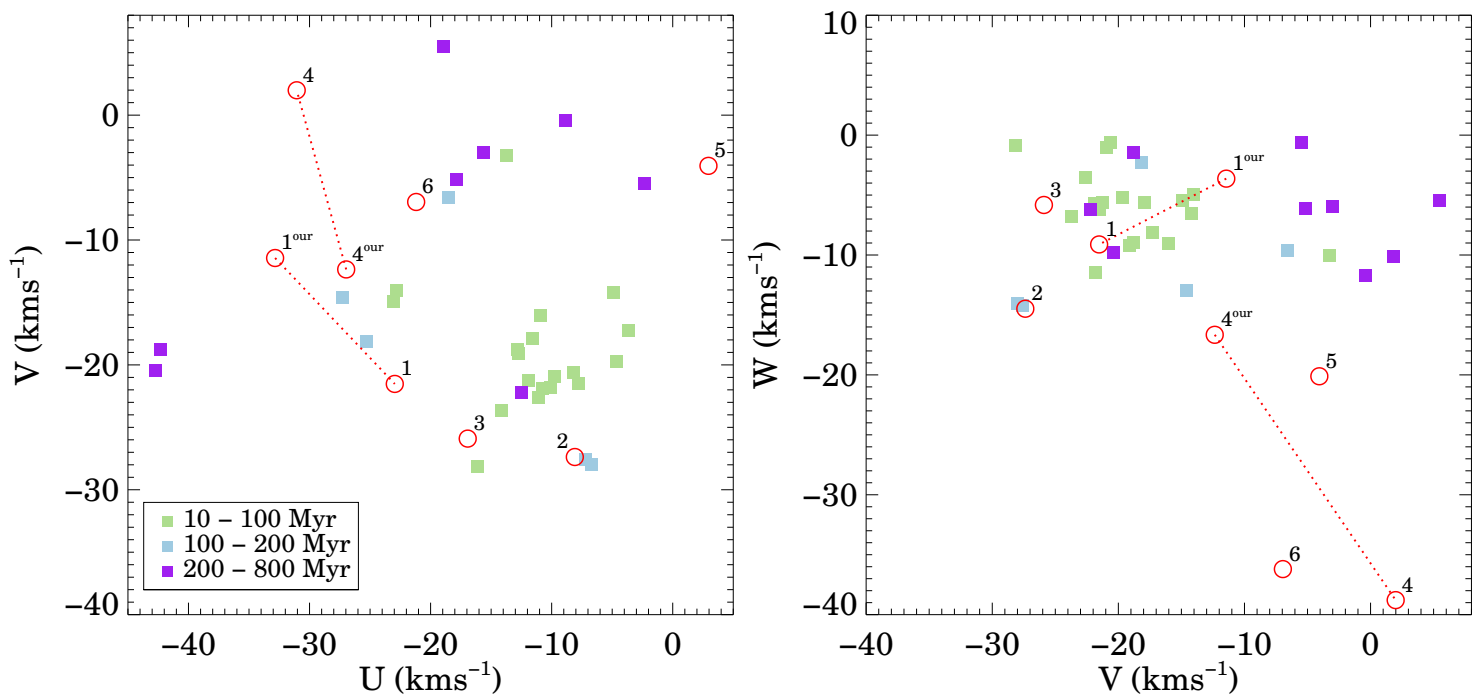

Figure 4. (U, V)- and (V, W)-planes for the host stars of extreme debris disks (red circles). For TYC 4515 and TYC 4946 , correspondingly to the measured $v_{\mathrm{r}}$ range, two U,V,W values are shown (Sect. 3.4). 1: TYC 4515, 2: TYC 5940, 3: TYC 8105, 4: TYC 4946, 5: TYC 4209, 6: TYC 4479. Filled squares display space velocities of known moving groups, associations, and open clusters located within 300 pc taken from the literature (Gagné et al. 2018; Soubiran et al. 2018). Different colors stand for different age ranges.

currently known values of the AB Dor group, making its membership unlikely.

According to our analysis, TYC 8105 has a wideseparation M5.5-type pre-main sequence companion. To determine its best-fit mass and isochrone age, we followed the Bayesian inference approach outlined in Pascucci et al. (2016) for the comparison of the measured $L_{*}$ and $T_{\text {eff }}$ parameters (from Table 3 ) with theoretical predictions from Baraffe et al. (2015). We obtained an isochrone age of $75_{-30}^{+50} \mathrm{Myr}$. Although somewhat lower, this age estimate is consistent with the lithium and gyro ages within the uncertainties. In fact, isochrones of Baraffe et al. (2015) are available only for solar metallicity, while TYC 8105 is a bit more metal rich at $[\mathrm{Fe} / \mathrm{H}] \sim 0.08$, which might lead to a slight underestimation of age. Moreover, isochronal model tracks tend to underestimate the age of low-mass pre-main sequence stars (e.g., Pecaut \& Mamajek 2016).

Taking into account the results of the different methods, we adopted ages of $120 \pm 20 \mathrm{Myr}$ and $130 \pm 30 \mathrm{Myr}$ for TYC 5940 and TYC 8105, respectively.

TYC 4209-falls between the Pleiades ( 120 Myr) and Hyades stars (600-800Myr old) in Fig. 2, and its measured $\mathrm{EW}_{L i}$ value makes it similar to members of the $200-300 \mathrm{Myr}$ old open cluster NGC 6475 (Villanova et al. 2009; Cummings \& Kalirai 2018; Bossini et al. 2019). The obtained $P_{\text {rot }}$ of 5.07 day also places the star between the sequences of Pleiades and Hyades/Praesepe in the period $-T_{\text {eff }}$ plot (Fig. 3 ). The average of the gyroage estimates is $295 \mathrm{Myr}$ with a range between 241 and $333 \mathrm{Myr}$. That this object is somewhat older than the previous two stars is also supported by its location in Fig. 4 outside the region of the young, 10-200 Myr old groups. Based on these results, we adopted an age of $275 \pm 50 \mathrm{Myr}$ for TYC 4209.

TYC 4515 and TYC 4946 - are the hottest stars in our sample. Studying the $\mathrm{Li}$ abundances in different open clusters showed that similar mid F-type members with effective temperatures between 6300 and $6900 \mathrm{~K}$ often have significant lithium depletion compared to co-eval but somewhat colder or hotter stars (e.g. Boesgaard \& Tripicco 1986; Balachandran 1995). This so-called Li dip has not been observed in the Pleiades, but according to Steinhauer \& Deliyannis (2004) it already appears at an age of $\sim 150 \mathrm{Myr}$. The measured $\mathrm{EW}_{L i}$ values for TYC 4946 and especially for TYC 4515 are significantly lower than those of Pleiades stars, implying a serious Li depletion and suggesting a lower limit of $150 \mathrm{Myr}$ for their ages. Although the galactic velocity components of both stars are quite uncertain due to the uncertainty of radial velocities (Sect. 3.3), in the case of TYC 4946, the possible $\mathrm{U}, \mathrm{V}, \mathrm{W}$ range mostly deviates from the one typical for nearby young stars (Fig. 4) further supporting this age estimate. Considering all results, we adopted a lower limit of $150 \mathrm{Myr}$ for both systems.

TYC 4479-has a lithium EW lower than those of the similar temperature members of the NGC 752 (1.3$1.8 \mathrm{Gyr}$ ) and roughly consistent with the upper limits measured for late G-type stars belonging to the $\sim 4 \mathrm{Gyr}$ 
old M67 open cluster (Fig. 2). In its TESS light curve, we found a variation with a period of $\sim 32$ days and an amplitude of $\sim 0.0012 \mathrm{mag}$. The observed period is slightly longer than the rotational periods observed for late G stars of M67 (Fig. 3). Assuming that the observed changes are also related to stellar rotation, the above mentioned gyrochronologic relations yield age estimates between 5.4 and $6.5 \mathrm{Gyr}$, with a mean of $6 \mathrm{Gyr}$, for TYC 4479. However, the calibrations of gyrochronology are based on well dated stars - typically members of open clusters and moving groups - whose metallicity differs only marginally from that of Sun, while TYC 4479 has an $[\mathrm{Fe} / \mathrm{H}]$ of -0.29 . According to Amard \& Matt (2020), this could have a significant effect: the rotation of such a metal-poor star is expected to be faster than a similar mass star with a solar metallicity. This can lead to an underestimation of the stellar age using the available gyrochronology relations. As Fig. 4 demonstrates, the $W$ velocity component of the star differs significantly from the typical W of nearby stellar groups younger than 800 Myr. By applying a recent age-velocity relation proposed by Almeida-Fernandes \& Rocha-Pinto (2018), we compiled a probability density function for the age of a star based on its UVW data. Based on this, the kinematic age is $4.8_{-1.6}^{+5.7} \mathrm{Gyr}$. All these results indicate that TYC 4479 is definitely older than 2 Gyr and its age is likely at least comparable to that of the Sun. For this star we adopted an age of $5 \pm 2$ Gyr.

All six systems are found to be older than $100 \mathrm{Myr}$, implying that the observed dust cannot be primordial material but rather is of secondary origin, i.e., these stars host extreme debris disks.

Using the obtained luminosity, effective temperature, and metallicity parameters (Tab. 1), we estimated the mass of the host stars based on the PARSEC evolutionary models (ver. 1.2S, Bressan et al. 2012). In this analysis, we took into account the estimated ages of the stars as a priori information. The resulting stellar masses are listed in Table 1. Using the same approach, we also estimated the masses of the companions. For TYC 4515B, TYC 4209B, and TYC 4479B, we used the PARSEC models, while for the late M-type TYC 8105B the evolutionary models were taken from Baraffe et al. (2015). For these objects, we assumed metallicities and ages similar to those of the primary components. The obtained stellar masses are listed in Table 3.

\section{CHARACTERIZATION OF THE DISKS}

\subsection{WISE observations}

The primary survey of WISE is comprised of three phases. In the first 7 months of the survey operation (between January and August of 2010) the satel- lite performed observations in all four bands (4-Band Cryo Phase) scanning the sky 1.2 times before the exhaustion of frozen hydrogen from its outer cryogen tank. This phase was followed by a short ( $\sim 2$ months) operational period when the focal plane was cooled by the inner cryogen system, allowing the scanning of $\sim 30 \%$ of the sky in the W1, W2, and W3 bands (3-Band Cryo Phase). Finally, after the end of the cryogenic mission, in the framework of the NEOWISE Post-Cryo survey phase approximately $70 \%$ of the sky was observed in the W1 and W2 bands achieving thereby a complete second coverage of the sky at the shortest wavelengths. At the close of this phase the satellite was put into hibernation in February 2011. In October 2013, the spacecraft was reactivated and sky surveys were continued in the W1 and W2 bands. The recent NEOWISE 2020 Data Release provides photometric data obtained in the first six years of the Reactivation survey, in which period the satellite scanned the sky approximately twelve times.

By gathering single exposure observations obtained for our targets during the above-mentioned survey phases from the NASA/IPAC Infrared Science Archive (IRSA) database, we could extract W1 and W2 photometric data clustered in 14-15 observational windows. Apart from a larger gap due to the hibernation period these windows are typically spaced by $\sim 6$ months. In the case of TYC 4479, these data are completed by W3 and W4 band photometry in the first two epochs. For the remaining sources, these long wavelength data are only available in the first epoch. Corresponding to the applied observing strategy of WISE (Wright et al. 2010), the individual observing windows of our targets are 0.734 days long with 10-300 single exposure measurements depending mainly on the ecliptic latitude of the source.

In the following analysis we used only the best quality single exposure data points and discarded measurements where the frameset images had low quality (qi_fact $=$ 0). Following the Explanatory Supplement ${ }^{9}$, in order to filter out framesets that could be contaminated by higher levels of charged particle hits or by scattered moon-light, we removed data points where the frameset was taken when the satellite was located within the nominal boundaries of the South Atlantic Anomaly $($ saa_sep $<5)$; or was performed within an area close to the Moon (moon_masked=1). We also removed all measurements having contamination and confusion flags (cc_flags) of either 'D', 'P', 'H', 'O', 'L', or 'R'. Moreover, we found that data points where the reduced $\chi^{2}$ of the profile-fit photometry measurement (w1rchi2 or

\footnotetext{
${ }^{9}$ wise2.ipac.caltech.edu/docs/release/allwise/expsup/
} 
w2rchi2) was higher than 5 were frequently outlying from the bulk of the data, and therefore we rejected them as well.

\subsection{Additional infrared data}

To expand our knowledge on the SED of the sources, we searched for additional infrared data in the literature. Using the IRAS Point Source (Helou et al. 1988) and Faint Source (FSC, Moshir 1990) catalogs, we found possible counterparts for four of our targets (TYC 4515, TYC 4209, TYC 8105, and TYC 5940) in the FSC. For TYC 4515, TYC 4209, and TYC 8105, the association with the nearby FSC source is unambiguous, since these targets are situated within the $1.5 \sigma$ IRAS error ellipse, and their WISE W3 band images show no other potentially contaminating objects in their environment (Fig. 1). Based on the IRAS error ellipse plotted over on the W3 band image (Fig. 1), in the case of TYC 5940, an additional fainter object, to the north-west of our target, can also contribute to the measured IRAS flux. TYC 8105 was detected both at 12 and $25 \mu \mathrm{m}$, for the other three sources, only $12 \mu \mathrm{m}$ photometry is available.

For TYC 4209, an additional data point at $9 \mu \mathrm{m}$ is available in the AKARI IRC catalog (Ishihara et al. 2010). TYC 4515 was serendipitously covered by a $24 \mu \mathrm{m}$ map obtained with the MIPS camera (Multiband Imaging Photometer for Spitzer, Rieke et al. 2004) (AOR: 15184640, PI: David Jewitt). In the latter case, MIPS images produced via the Enhanced Basic Calibrated Data (EBCD) pipeline (version S18.12.0) were downloaded from the Spitzer Heritage Archive and the MOPEX software package (MOsaicking and Point source Extraction, Makovoz \& Marleau 2005) was used to create a mosaic from the individual frames. Then point spread function (PSF) photometry was applied to obtain the flux density of TYC 4515. The source has a positional offset of $00^{\prime \prime} 55$ with respect to the proper motion corrected Gaia EDR3 position of the star. The final photometric uncertainty was derived as the quadratic sum of the measurement noise and the conservative absolute calibrational uncertainty of the MIPS $24 \mu \mathrm{m}$ array $\left(4 \%\right.$, MIPS Instrument Handbook $\left.{ }^{10}\right)$. As a result, we obtained a flux density of $25.2 \pm 1.4 \mathrm{mJy}$ at $24 \mu \mathrm{m}$.

TYC 4479, which is located in the foreground of the Cep OB4, was observed at 3.6 and $4.5 \mu \mathrm{m}$ in a mapping project that targeted this association with the IRAC intrument of Spitzer (AOR: 68515840, PI: Joseph Hora). We performed aperture photometry using those six 'corrected BCD' (cBCD) images that cover our target. The

10 https://irsa.ipac.caltech.edu/data/SPITZER/docs/mips/ mipsinstrumenthandbook/ aperture radius was set to 3 pixels (3!'66), the sky background was computed in an annulus between 12 pixels and 20 pixels. The background was estimated using an iterative sigma-clipping method, where the threshold was set to $3 \sigma$. We applied aperture correction with a factor of 1.113 (IRAC Instrument Handbook ${ }^{11}$ ) in both filters. Array location- and pixel phase-dependent photometric corrections were also done using the recipe from the IRAC Instrument Handbook. Finally, the six flux density values obtained in the different frames were averaged to get the final photometry. This approach yielded flux densities of $43.6 \pm 0.9 \mathrm{mJy}$ and $29.9 \pm 0.6 \mathrm{mJy}$ at $3.6 \mu \mathrm{m}$ and $4.5 \mu \mathrm{m}$, respectively. The quoted uncertainties are quadratic sums of the measurement errors and the absolute calibrational uncertainty (2\%, IRAC Documentation ${ }^{12}$ ).

\subsection{Disk properties}

To estimate the basic disk properties, we fitted a single temperature blackbody model to the observed IR excess calculated as the difference between the measured flux densities and the predicted photosphere. MidIR light curves showed evidence of variability at several EDDs (Melis et al. 2012; Meng et al. 2012, 2015; $\mathrm{Su}$ et al. 2019b). To avoid combining data points potentially related to different disk states, for the fitting process, we used only the weighted averages of W1-W4 band WISE single exposure photometry obtained in the first observing window of the satellite's primary mission. While the AllWISE catalog combines data from the WISE cryogenic and NEOWISE post-cryogenic survey phases, our selected measurements form a homogeneous and simultaneously obtained data set in all four WISE filters. The uncertainties of the measured flux densities were calculated as the quadratic sum of the calibration errors (WISE All-Sky Release Explanatory Supplement Sect. $4.4^{13}$ ) and the errors of the weighted averages. In the computation of uncertainties of the excesses, the uncertainties of the predicted photospheric fluxes were estimated to be $3 \%$ and added quadratically to the previously derived errors.

Since the SED shapes differ from the $F_{\nu} \propto \nu^{-2}$ reference spectrum used in the WISE catalog, the measured flux densities need to be color corrected. The color correction factors $\left(f_{\mathrm{cc}}\right)$ were computed based on a simple

\footnotetext{
11 https://irsa.ipac.caltech.edu/data/SPITZER/docs/irac/ iracinstrumenthandbook/

12 https://irsa.ipac.caltech.edu/data/SPITZER/docs/irac/ warmimgcharacteristics/

13 http://wise2.ipac.caltech.edu/docs/release/allsky/expsup/sec4_ 4h.html\#CalibrationU
} 


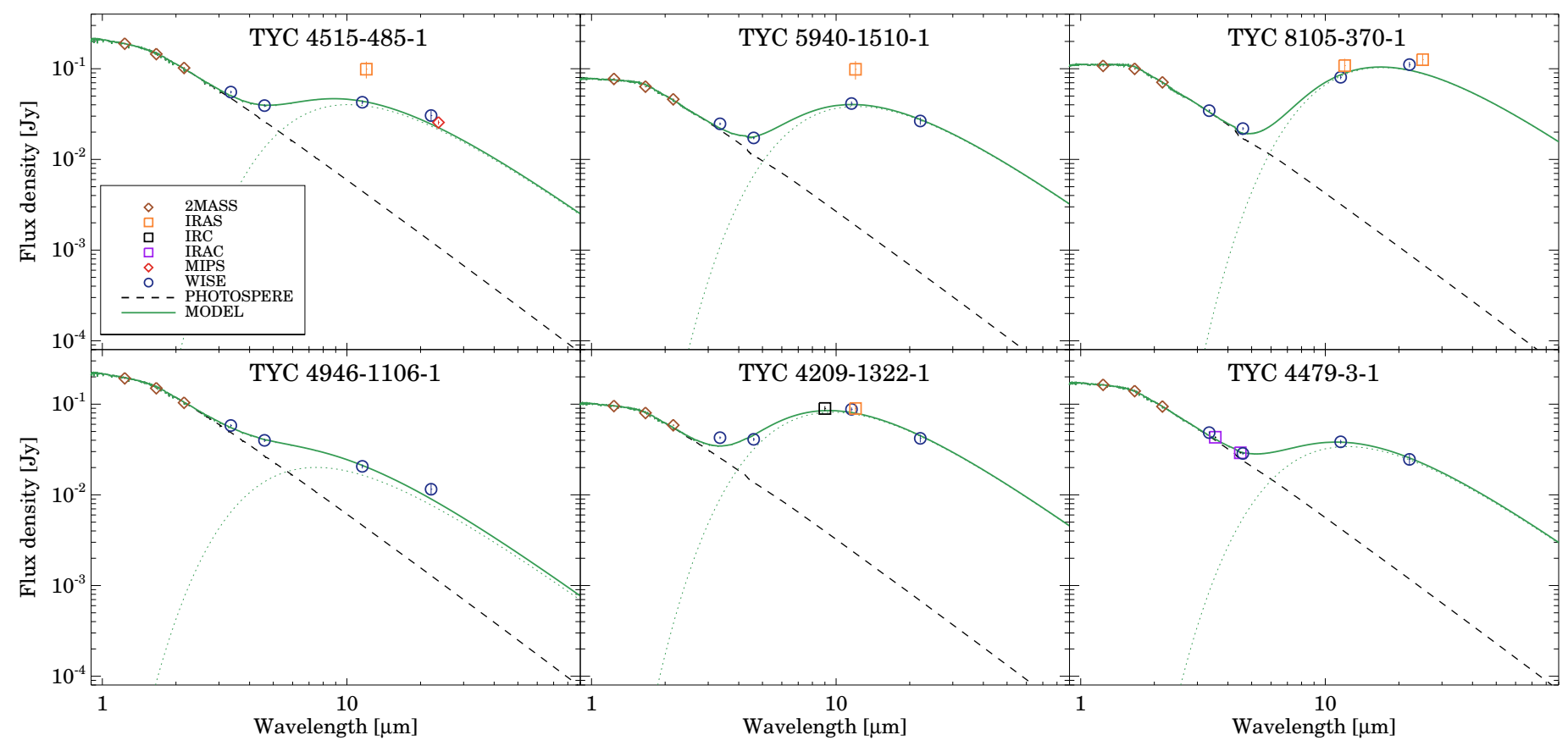

Figure 5. Color corrected SEDs overplotted with the photosphere and disk models. Model parameters are given in Table 1.

SED model (derived as the sum of the stellar photosphere and a blackbody fitted to the excess computed from the original data). The $f_{\mathrm{cc}}$ values were less than $1 \%$, except the wide bandpass $\mathrm{W} 3$ measurements, where they could be as high as 10\%. The color corrected WISE flux densities are plotted in Figure 5.

The SED of the disk was computed as the excess above the photosphere (Fig. 5). In order to select the best-fitting blackbody model, we applied a LevenbergMarquardt algorithm (Markwardt 2009) using the characteristic dust temperature and the solid angle of the emitting region as free parameters. Color correction of other available IR data points (Sect. 4.2) was also performed using the above models. Table 4 summarizes the color corrected infrared photometric data for all six objects. The obtained dust temperatures and fractional dust luminosities are listed in Table 1. Temperatures fall between 300 and $680 \mathrm{~K}$, while fractional luminosities range from 0.01 to 0.07 . Figure 5 shows, that the WISE data points at $3.4 \mu \mathrm{m}$ are systematically higher than the model fit. In the case of TYC 4209, the measured flux density in the W1 band is $5.5 \sigma$ higher than the model fit, which likely indicates the presence of a hotter dust component in the disk. For the rest of the sample, the deviations between the measured fluxes and the models are less than $3 \sigma$. Nevertheless, it cannot be excluded that some of these disks contain additional even hotter dust as also suggested by color temperatures derived from W1, W2 data (see Fig. 7).
Assuming an optically thin circumstellar dust ring with grains radiating like blackbodies, the ring radius can be calculated from the dust temperature and the stellar luminosity as $R_{\mathrm{bb}}[\mathrm{au}]=\left(\frac{L_{\mathrm{star}}}{L_{\odot}}\right)^{0.5}\left(\frac{278 K}{T_{\text {dust }}}\right)^{2}$. The derived blackbody radii range from $\sim 0.3$ to 0.7 au (Table 1).

Several caveats are associated with our simple approach. Extreme debris disks tend to show strong solid state emission features (Olofsson et al. 2012). Without any mid-IR spectral information, we do not know how the presence of such features modifies the shape of the SED. Furthermore, in the estimation of disk radii, we assumed large grains, behaving as blackbodies, and did not account for smaller particles that are ineffective emitters and thus become hotter compared to larger grains at the same location. The derived high fractional luminosity values $(\gtrsim 0.01)$ indicate that the dust may be at least partly optically thick along the line of sight, which was also not considered in our simple model.

\section{DISK VARIABILITY}

The available infrared data allow us to assess the mid-IR variability of the sources on several different timescales. Using single exposure WISE data, we searched for flux changes on daily and annual timescales. For those sources where additional earlier IR photometry is available (IRAS, AKARI, Spitzer) we could also investigate variability on decadal timescales. 
Table 4. Measured and predicted flux densities.

\begin{tabular}{|c|c|c|c|c|c|}
\hline Name & Instr. & Epoch & $\begin{array}{c}\lambda \\
(\mu \mathrm{m})\end{array}$ & $\begin{array}{c}P_{\nu}^{a} \\
(\mathrm{mJy})\end{array}$ & $\begin{array}{c}F_{\nu}{ }^{b} \\
(\mathrm{mJy})\end{array}$ \\
\hline \multirow[t]{6}{*}{ TYC 4515} & WISE & 2010 & 3.35 & 47.3 & $55.3 \pm 1.7$ \\
\hline & WISE & 2010 & 4.60 & 26.1 & $39.2 \pm 1.3$ \\
\hline & WISE & 2010 & 11.56 & 4.5 & $42.7 \pm 2.1$ \\
\hline & IRAS & 1983 & 12.00 & 4.1 & $98.9 \pm 20.8$ \\
\hline & WISE & 2010 & 22.09 & 1.2 & $30.5 \pm 3.7$ \\
\hline & MIPS & 2006 & 23.67 & 1.1 & $25.6 \pm 1.4$ \\
\hline \multirow[t]{5}{*}{ TYC 5940} & WISE & 2010 & 3.35 & 21.0 & $24.7 \pm 0.8$ \\
\hline & WISE & 2010 & 4.60 & 11.0 & $17.3 \pm 0.6$ \\
\hline & WISE & 2010 & 11.56 & 2.0 & $41.3 \pm 2.3$ \\
\hline & IRAS & 1983 & 12.00 & 1.9 & $98.6 \pm 22.7$ \\
\hline & WISE & 2010 & 22.09 & 0.6 & $26.5 \pm 2.4$ \\
\hline \multirow[t]{6}{*}{ TYC 8105} & WISE & 2010 & 3.35 & 33.3 & $34.5 \pm 1.2$ \\
\hline & WISE & 2010 & 4.60 & 16.8 & $21.9 \pm 0.8$ \\
\hline & WISE & 2010 & 11.56 & 3.2 & $80.5 \pm 3.9$ \\
\hline & IRAS & 1983 & 12.00 & 3.0 & $108.2 \pm 20.6$ \\
\hline & WISE & 2010 & 22.09 & 0.9 & $111.0 \pm 7.6$ \\
\hline & IRAS & 1983 & 25.00 & 0.7 & $125.9 \pm 18.9$ \\
\hline \multirow[t]{4}{*}{ TYC 4946} & WISE & 2010 & 3.35 & 48.8 & $58.2 \pm 1.9$ \\
\hline & WISE & 2010 & 4.60 & 26.8 & $39.9 \pm 1.6$ \\
\hline & WISE & 2010 & 11.56 & 4.6 & $20.6 \pm 1.1$ \\
\hline & WISE & 2010 & 22.09 & 1.3 & $11.5 \pm 1.6$ \\
\hline \multirow[t]{6}{*}{ TYC 4209} & WISE & 2010 & 3.35 & 25.6 & $42.7 \pm 1.4$ \\
\hline & WISE & 2010 & 4.60 & 13.6 & $40.9 \pm 1.6$ \\
\hline & IRC & 2006 & 9.00 & 4.0 & $89.6 \pm 5.5$ \\
\hline & WISE & 2010 & 11.56 & 2.4 & $87.7 \pm 4.2$ \\
\hline & IRAS & 1983 & 12.00 & 2.3 & $89.5 \pm 12.5$ \\
\hline & WISE & 2010 & 22.09 & 0.7 & $41.9 \pm 4.1$ \\
\hline \multirow[t]{6}{*}{ TYC 4479} & WISE & 2010 & 3.35 & 45.8 & $48.4 \pm 1.6$ \\
\hline & IRAC & 2019 & 3.55 & 41.3 & $43.1 \pm 0.9$ \\
\hline & IRAC & 2019 & 4.49 & 25.5 & $29.0 \pm 0.6$ \\
\hline & WISE & 2010 & 4.60 & 24.2 & $28.5 \pm 1.0$ \\
\hline & WISE & 2010 & 11.56 & 4.4 & $38.4 \pm 1.9$ \\
\hline & WISE & 2010 & 22.09 & 1.2 & $24.6 \pm 3.5$ \\
\hline
\end{tabular}

${ }^{a}$ Predicted photospheric flux densities.

${ }^{b}$ Measured flux densities. The quoted values are color corrected.

\subsection{Exploring the annual variability in $W 1 / W 2$}

To search for flux changes occuring on timescales of years, we used all single exposure $\mathrm{W} 1$ and $\mathrm{W} 2$ band data points not discarded in the quality check. We employed two different discriminants: the correlation-based Stetson $J$ index $\left(\mathrm{S}_{J}\right.$, Stetson 1996) and the scatter-based $\chi^{2}$ test (Sokolovsky et al. 2017). By quantifying correlation of variability in two or more bands, the Stetson $J$ index provides a robust metric to search for brightness changes. Involving the $\mathrm{W} 1$ and $\mathrm{W} 2$ bands, this index can be computed as:

$$
S_{J}=\frac{\sum_{k=1}^{n} w_{k} \operatorname{sgn}\left(P_{k}\right) \sqrt{\left|P_{k}\right|}}{\sum_{k=1}^{n} w_{k}}
$$

where $n$ is the number of paired observations taken at the same time, $w_{k}$ is the weight of the $k$-th epoch (set to 1 uniformly in our case). $P_{k}$ is the product of the normalized residuals of two observations, $P_{k}=\left(\sqrt{\frac{n}{n-1}} \frac{W_{1, k}-\bar{W}_{1}}{\sigma_{W_{1, k}}}\right)\left(\sqrt{\frac{n}{n-1}} \frac{W_{2, k}-\bar{W}_{2}}{\sigma_{W_{2, k}}}\right)$, where $W_{1, k}$ and $W_{2, k}$ are the $k$-th measured magnitudes in the given bands, $\sigma_{W_{1, k}}$ and $\sigma_{W_{2, k}}$ are their uncertainties, while $\bar{W}_{1}$ and $\bar{W}_{2}$ are the means of the measured data points in the given bands. We also searched for variability in individual bands by computing the value of $\chi_{\text {red }}^{2}$ as

$$
\chi_{\text {red }}^{2}=\frac{1}{N-1} \sum_{k=1}^{n}\left(\frac{W_{k}-\bar{W}}{\sigma_{W_{k}}}\right)^{2},
$$

where $W_{k}$ and $\sigma_{W_{k}}$ are the measured magnitudes and their uncertainties in the specific band.

To define $S_{J}$ and $\chi_{\text {red }}^{2}$ cutoff values for separating variable objects from non-variable ones, we used an empirical approach. We selected all objects from the AllWISE catalog that 1 ) are located within $2^{\circ}$ of our targets (thus have similar time sampling), 2) have W2 band magnitudes within \pm 1.0 mag to that of the specific EDD, and 3) are not marked by any contamination flag in the W1 and W2 bands ('cc_flg' $=0$ ). We gathered all single exposure photometric data for the selected objects and compiled their light curves by applying the same quality criteria as for our targets (see Sect. 4.1). Finally we computed the $S_{J}$ and $\chi_{\text {red }}^{2}$ values for the light curves and compiled the histograms of variability indices for all studied regions independently. The obtained histograms typically show asymmetric behavior with a tail at positive values (see, e.g., Fig. 6 for the case of TYC 5940). Supposing that the majority of objects show no significant variations, this finding is consistent with our expectations. Variability in individual bands is expected to be accompanied with higher $\chi_{\text {red }}^{2}$ values. For non-variable objects with random noise, no correlation is expected between the different band observations thus the Stetson index should be close to zero, while in the case of the real correlated variability the index should be positive. To derive the mean and standard deviation of the obtained distributions $\left(\bar{D}, \sigma_{D}\right)$ we performed an iterative $3 \sigma$ clipping process. Then the cutoff value was computed as $\bar{D}+5 \sigma_{D}$ (Fig. 6 ).

We found that the cutoff values differ from region to region, they range from 0.20 to 0.42 for the Stetson index, from 1.09 to 1.53 for the $\chi_{\text {red,W1}}^{2}$, and 1.74 to 2.58 for the $\chi_{\text {red,W2 }}^{2}$ index. Interestingly, the means of the $\mathrm{S}_{J}$ distributions were found to be larger than zero $(0.08-0.1)$ in all cases. This is in line with the result of Secrest \& Satyapal (2020), who, examining the mid-IR variability of dwarf galaxies, found that the distribution 
of Pearson correlation coefficients between WISE W1 and W2 band photometry follows a normal distribution that is slightly shifted toward positive values (with a mean of 0.06 and a standard deviation of 0.09 ). Table 5 lists the obtained variability indices and the relevant critical cutoff values, the latter ones are in brackets. We identified four objects - TYC 8105, TYC 5940, TYC 4209, and TYC 4479 - that show significant variability. While the variation of TYC 8105 is limited to the W2 band, the other three systems exhibit flux changes in both WISE bands in a correlated way. The light curves of TYC 4515 and TYC 4946 display no significant variability. By repeating the above procedure using only data points obtained in the NEOWISE Reactivation phase would not change our conclusions on the variability of the targets (see Table 5).

Table 5. Variability indices

\begin{tabular}{c|cccc}
\hline \hline Name & $\chi_{\text {red,W1 }}$ & $\chi_{\text {red,W2 }}$ & $S_{\text {J }}$ & $\chi_{\text {red,W1-W2 }}$ \\
\hline \multicolumn{5}{c}{ AllWISE + NEOWISE Reactivation data } \\
TYC 4515 & $0.99(1.47)$ & $1.76(1.83)$ & $0.21(0.36)$ & $1.08(1.48)$ \\
TYC 5940 & $2.78(1.53)$ & $7.61(1.93)$ & $1.08(0.38)$ & $2.15(1.55)$ \\
TYC 8105 & $1.25(1.45)$ & $2.51(1.82)$ & $0.20(0.32)$ & $1.48(1.49)$ \\
TYC 4946 & $1.06(1.48)$ & $1.69(2.58)$ & $0.31(0.42)$ & $0.98(1.72)$ \\
TYC 4209 & $19.18(1.09)$ & $105.20(1.74)$ & $5.72(0.20)$ & $14.52(1.19)$ \\
TYC 4479 & $2.37(1.44)$ & $8.96(2.21)$ & $1.08(0.37)$ & $2.10(1.60)$ \\
& NEOWISE Reactivation only & \\
TYC 4515 & $0.86(1.44)$ & $1.66(1.98)$ & $0.27(0.40)$ & $0.88(1.49)$ \\
TYC 5940 & $2.98(1.49)$ & $7.86(2.01)$ & $1.34(0.40)$ & $1.82(1.56)$ \\
TYC 8105 & $1.19(1.30)$ & $1.99(1.77)$ & $0.24(0.32)$ & $1.19(1.36)$ \\
TYC 4946 & $1.01(1.37)$ & $1.50(2.34)$ & $0.25(0.41)$ & $0.92(1.61)$ \\
TYC 4209 & $20.46(0.97)$ & $113.04(1.59)$ & $6.05(0.19)$ & $15.56(1.09)$ \\
TYC 4479 & $2.66(1.35)$ & $10.66(1.93)$ & $1.34(0.36)$ & $2.32(1.48)$ \\
\hline
\end{tabular}

We also examined whether the W1-W2 color index of our targets showed any significant variations during the WISE observations. By calculating the $\chi_{\text {red,W1-W2 val- }}^{2}$ ues for our targets and for their comparison samples, and then using the above technique to set the thresholds, we found that the W1-W2 colors of TYC 5940, TYC 4209, and TYC 4479 changed significantly between 2010 and 2019.

Figure 7 shows how the disk fluxes in the W1 and W2 bands - computed as the difference of the measured single exposure photometry and the photospheric flux densities - of our targets changed between 2010 and 2019. In addition to the individual data points, their averages in each observing window (seasonal averages) were also plotted. In the course of averaging, an iterative sigma clipping algorithm was utilized where the clipping was set to $3 \times$ of the standard deviation. Formal uncertainties were computed from the dispersion of data
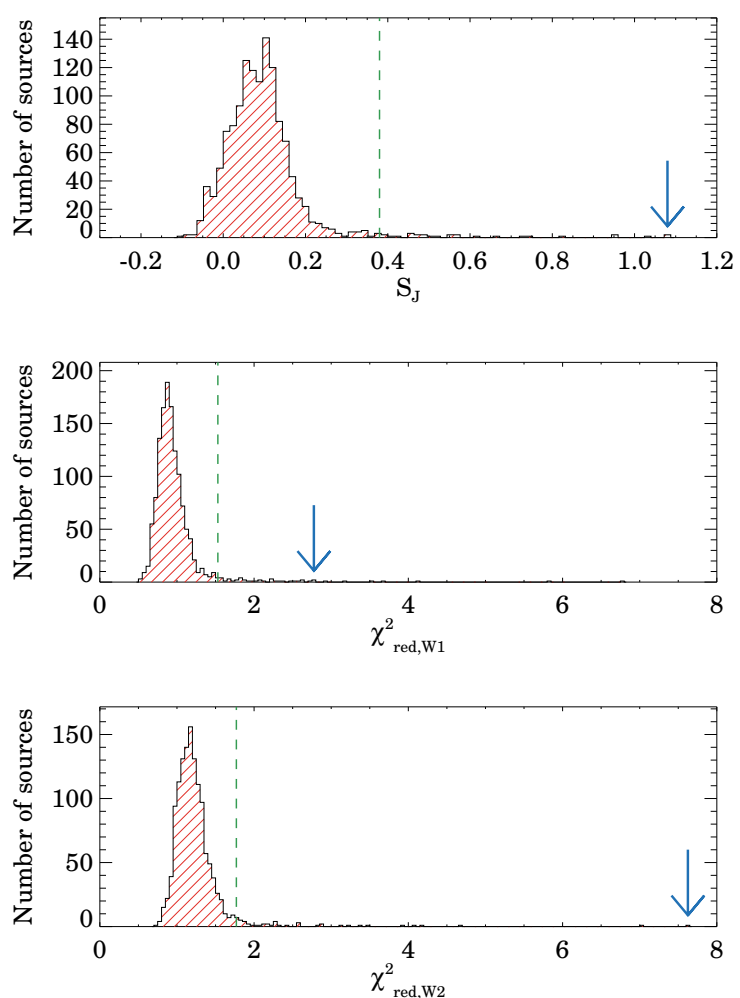

Figure 6. Histogram of the Stetson, $\chi_{\text {red,W1}}^{2}$, and $\chi_{\text {red,W2 }}^{2}$ variability indices computed for WISE sources in the vicinity of TYC 5940. Blue arrows show index values obtained for TYC 5940, while vertical dashed green lines represent the derived cutoff values for the discrimination of nonvariable/variable objects (see Sect. 5).

points involved in the averaging. Ratios of the $\mathrm{W} 1$ and W2 band disk fluxes as well as the corresponding color temperatures were also derived and plotted in separate panels.

The four variable objects are displayed in the first four panels of Fig. 7. TYC 5940 exhibited significant variability in both WISE bands. Following an initial drop, in 2014 the object started brightening, and the disk flux increased by $78 \%$ in $\mathrm{W} 1$ and by $54 \%$ in W2 over six years. TYC 8105 displayed less pronounced variations, with no long term trend in the flux changes. According to our analysis, only the W2 band variability is formally significant, with a peak-to-peak amplitude of about $34 \%$. The disk of TYC 4209 showed very prominent flux changes. After an intermediate flux level in 2010, its flux considerably dropped by 2014, which was followed by a dramatic brightening on a timescale of about one year. At 3.4 and $4.6 \mu \mathrm{m}$ the disk became brighter by about $56 \%$ and $64 \%$, respectively. Over the next one year the disk flux levels remained nearly constant, but after that a significant fading started. By late 2017, the disk reached the flux 
level just preceding the brightening phase in 2014. The light curve between 2014 and 2017 was rather symmetric, the pace of rising and fading was roughly similar. From 2018 the disk started brightening again. TYC 4479 was constant between 2010 and 2015. In early 2016, an abrupt flux rise by a factor of 2 occurred in less than 6 months. The peak was immediately followed by an exponential-like fading. By 2019, the source returned to its normal flux level, thus this asymmetric brightening event lasted for about 2.5 years. The last WISE observation in 2019 August showed a higher flux level again. TYC 4515 and TYC 4946 exhibited no variability complying with the criteria of formal significance in our analysis. Nevertheless, the light curves of TYC 4946 suggest a wave-like pattern between 2014 and 2019, repeated in both the W1 and W2 bands.

\subsection{Optical variability of the host stars}

In the calculation of infrared excesses we assumed that the host stars did not exhibit variability trends on the timescales of our WISE light curves in Fig. 7. To evaluate this assumption and to exclude that the observed mid-IR flux changes could be driven by stellar variations, we examined the long term optical light curves of the host stars of the four variable objects. The data were taken from the ASAS-SN survey (Shappee et al. 2014; Kochanek et al. 2017) that provides V-band photometry for the whole sky with a cadence of $\sim 2-3$ days. These measurements allowed us to investigate the optical properties over only a part of the NEOWISE Reactivation mission (Fig. 7). However, they are still relevant since the specific disks showed significant changes in mid-IR wavelengths during this period as well.

Our analysis showed that all monitored stars were stable, their light curves were flat with root mean square (rms) noise <0.02 magnitude. In the same time intervals, TYC 5904, TYC 4209, and TYC 4479 displayed peak-to-peak variations of $0.14,0.39$, and $0.17 \mathrm{mag}$ in the W2 band averaged photometry, which thus cannot be explained with variable stellar radiation. TYC 8105 shows a more modest change in the W2 band with a peak-to-peak change of $0.07 \mathrm{mag}$ during the NEOWISE Reactivation mission. Based on TESS data (Appendix B), within our sample, this star exhibits the largest rotational modulation. However, even in this case the amplitude is only $0.019 \mathrm{mag}$. Moreover, the length of the WISE observing windows are between 1.9 and 7.3 days for this object, thus our averaging process within the windows mostly cancels the effect of the rotational variability, which has a period of $\sim 5$ days. A further indication that the observed W2 band change is associated to the disk is that the measured peak-to- peak change in the same time period in the W1 band - where the disk's contribution is negligible - was only 0.022 mag.

\subsection{Exploring the hourly/daily variations}

To examine disk flux variations on shorter hourly/daily timescales, we turn to WISE single exposures performed within a specific observational window. The length of individual data sets in these windows depends on the target's sky position. Due to its high ecliptic latitude of $\sim 87^{\circ}$, TYC 4209 was observed for 17-34 days at a time. The other five targets have typical coverages shorter than a week. To identify objects showing significant variability on these shorter timescales, we used the same strategy as we described in Sect. 5.1, i.e. the variability indices $\left(S_{\mathrm{J}}\right.$ and $\chi_{\text {red }}^{2}$ values) computed for our target were compared to those of nearby similarly bright sources that are supposed to be mostly non-variable.

In the W1 and W2 bands, where photometry was available for all epochs, we found significant variability only in one source, TYC 4209, in two observational windows (2015 Oct 19 - Nov 5; 2017 April 8 - May 11; marked in Fig. 7) in both bands. Note that this source has by far the broadest observing window in our sample. The WISE light curves are displayed in Fig. 8, showing both the individual single exposures and tenpoint binned values. In both windows, a fading trend can be recognized. The observed trends were characterized by fitting a straight line to the binned data considering their error bars. These yielded slopes of $b_{W 1}=-53 \pm 11 \mathrm{mJy} \mathrm{yr}^{-1}$ and $b_{W 2}=-75 \pm 11 \mathrm{mJy} \mathrm{yr}^{-1}$ in the first window and $b_{W 1}=-17 \pm 4 \mathrm{mJy} \mathrm{yr}^{-1}$ and

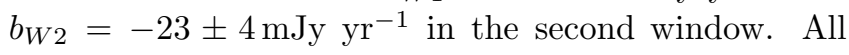
obtained slopes are significantly different from zero. We compared these slopes with the long term flux changes in Fig. 7. In the first window, our result is consistent with the fading since the previous window, but its pace is much higher. Extrapolating the rapid fading would result in the disappearance of the excess on a timescale of $\sim 0.5$ year. In contrast to this, the source became brighter by the next window $\sim 6$ months later. In the second window, the observed trend qualitatively matches the long term fading behavior, however the fading rate is faster. Similar few weeks long rapid variations are seen in Spitzer light curves of other EDDs (ID 8, P 1121) of better sampling ( $\mathrm{Su}$ et al. 2019b). In those objects, the quasi-periodic changes were found and interpreted as orbital evolution of an optically thick dust cloud ( $\mathrm{Su}$ et al. 2019b).

In the W3/W4 bands, our data are limited to 1-2 observing windows in 2010-11. Using the same strategy 

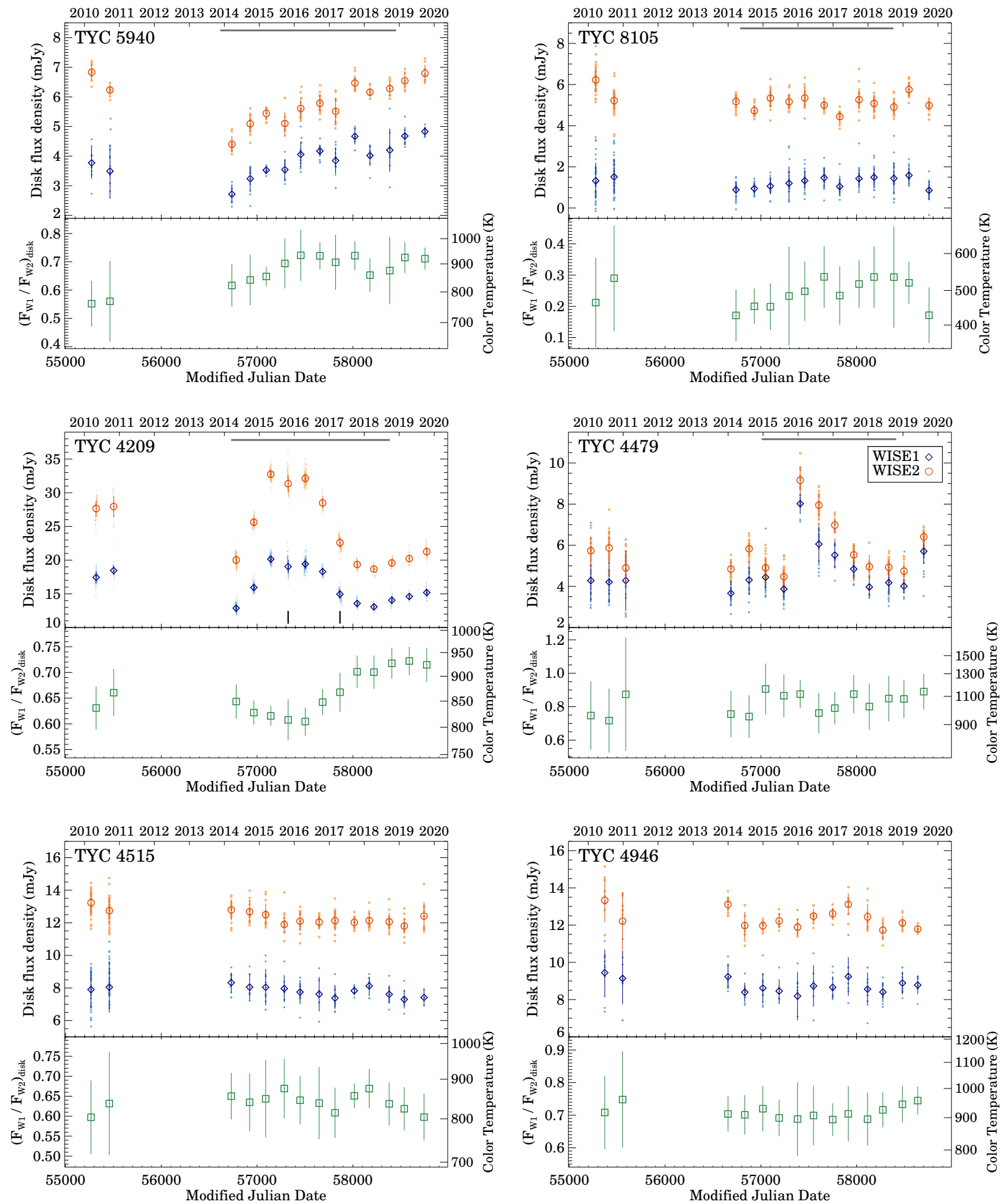

Figure 7. WISE W1 $(3.4 \mu \mathrm{m})$ and W2 $(4.6 \mu \mathrm{m})$ band disk fluxes (the measured excess emissions) for our targets between 2010 and 2019. Small dots show single exposure data points, while larger symbols with error bars denote the seasonal averages. Horizontal gray lines show the time ranges of available ASAS-SN photometric observations (Sect. 5.2). Small vertical lines in the plot of TYC 4209 mark those observational windows in which significant daily disk flux changes have been detected (Sect. 5.3). Ratios of W1 to W2 band seasonal averages are also shown (bottom panels) together with the corresponding color temperatures. 

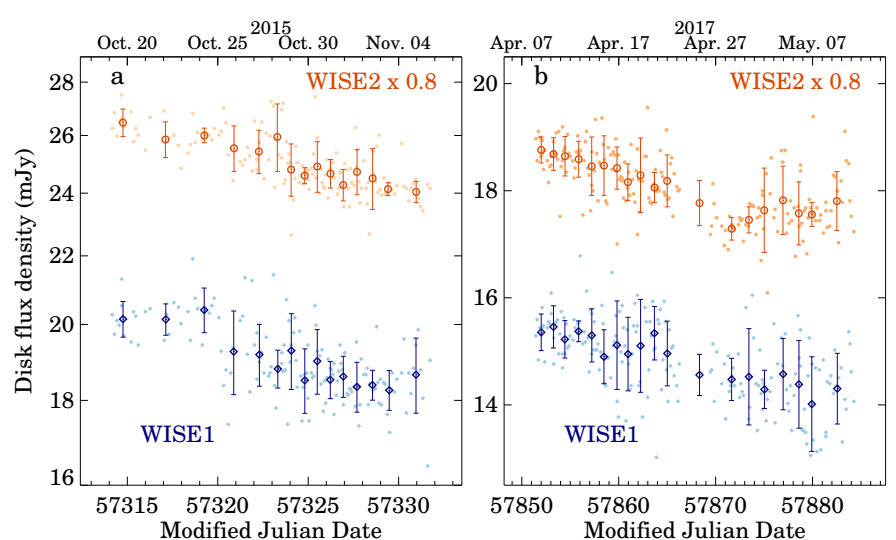

Figure 8. WISE W1/W2 band disk fluxes of TYC 4209 in the observational windows when significant flux changes were detected (Sect. 5.3). For better visualization, the W2 light curve was scaled by 0.8 .

as above, we found no significant daily variability in any of the sources.

\subsection{Search for decadal changes}

For five objects, additional IR photometry is available from different space missions (Table 4). In those four cases where IRAS measurements also exist, the data cover $\sim 30$ years. These additional data allow us to check for long-term variability. Fig. 5 shows the data points, together with the WISE photometry and the models fitted to the WISE data (Sect. 4.3). In most cases, the additional data match the model curves well. In TYC 4515 and TYC 5940, the IRAS $12 \mu \mathrm{m}$ data points exceed the models, however, even in these cases the significance of the excess remains below $3 \sigma$. Moreover, in TYC 5940 the $12 \mu \mathrm{m}$ excess might be contaminated by a nearby source (Sect. 4.2). Thus, the available observations do not support any long-term IR variability exceeding the formal uncertainties in our sample.

\section{DISCUSSION}

\subsection{Steady-state or transient dust production?}

As a first step in analyzing the nature and origin of the observed abundant warm debris material around our targets, we examined whether it could be produced in a steady-state grind down of an in situ planetesimal belt, a massive exosolar analog of our Solar System's asteroid belt. If located close to the star, the evolution of such inner planetesimal belts is very rapid due to the short collisional timescale. Based on the analytical steady state evolutionary model of Wyatt et al. (2007), at any given age there is a maximum possible fractional luminosity $\left(f_{\mathrm{d}, \max }\right)$ of a debris ring. Following Wyatt et al. (2007) we adopted a belt width of $d r=0.5 r$, a maximum as- teroid size of $2000 \mathrm{~km}$, typical planetesimal strength of $Q_{D}^{*}=200 \mathrm{~J} \mathrm{~kg}^{-1}$, and planetesimal eccentricity of 0.05 , which resulted in

$$
\begin{aligned}
f_{\mathrm{d}, \max }=1.6 \times 10^{-4} & \left(\frac{R_{\text {disk }}}{1 \mathrm{AU}}\right)^{7 / 3}\left(\frac{M_{*}}{M_{\odot}}\right)^{-5 / 6} \\
& \left(\frac{L_{*}}{L_{\odot}}\right)^{-1 / 2}\left(\frac{t}{\mathrm{Myr}}\right)^{-1} .
\end{aligned}
$$

Wyatt et al. (2007) argued that, even by considering the uncertainties in their model, debris rings with $f_{\mathrm{d}} / f_{\mathrm{d} \text {,max }}$ ratios higher than 1000 could not be produced through steady-state processes, but should be linked to some transient event instead. For computing $f_{\mathrm{d} \text {,max }}$, stellar and disk parameters were taken from Table 1. By assuming that the collisional cascade started early, for $t$ we adopted the age of the systems (Table 1). For our targets, the ratios of the measured fractional luminosities to the theoretical maxima (Table 1) range between $\sim 8 \times 10^{4}$ and $2.6 \times 10^{6}$. We note, however, that $f_{\mathrm{d} \text {, max }}$ strongly depends on the disk radius which is not reliably known. In Sect. 4.3 we used a simple blackbody model, that can lead to an underestimation of the disk radii. Based on debris disks spatially resolved by the Herschel Space Observatory, in systems with Sun-like host stars, the ratios of the true disk radii to the blackbody radii are $<5$ (fig. 4b in Pawellek et al. 2014). Entering $5 \times$ larger radius values into the equation results in $\sim 43 \times$ higher $f_{\mathrm{d}, \max }$ values. However, even this way we still get $f_{d} / f_{\mathrm{d} \text {,max }}>1800$ for all sources, implying they are probably experiencing a transient phase.

\subsection{The origin of disk variability}

Figure 7 shows that in most objects, parallel to the detected mid-IR flux changes, the color temperature derived from the ratio of the $3.4 \mu \mathrm{m}$ and $4.6 \mu \mathrm{m}$ fluxes was also variable. In order to identify the physical processes behind these variations, we correlated the $\mathrm{W} 1$ and $\mathrm{W} 2$ multiepoch fluxes, by plotting the seasonal averages in Fig. 9. The photometric point distributions were then confronted with the predictions of two simple models: the first one assumes that the temperature of the circumstellar dust grains can change in time, while the second one keeps the temperatures constant but varies the total emitting dust surface area. Variability caused by the latter process would appear in Fig. 9 as a straight line whose slope is related to the invariable temperature; while a temperature rise in the first model would outline a curve starting from the origin.

The distribution of the WISE data points of TYC 4479 in Fig. 9 (left) is consistent with a line crossing the origin within the formal fit uncertainties. The derived $3.4 / 4.6 \mu \mathrm{m}$ color temperature is $1060 \mathrm{~K}$. The points are 

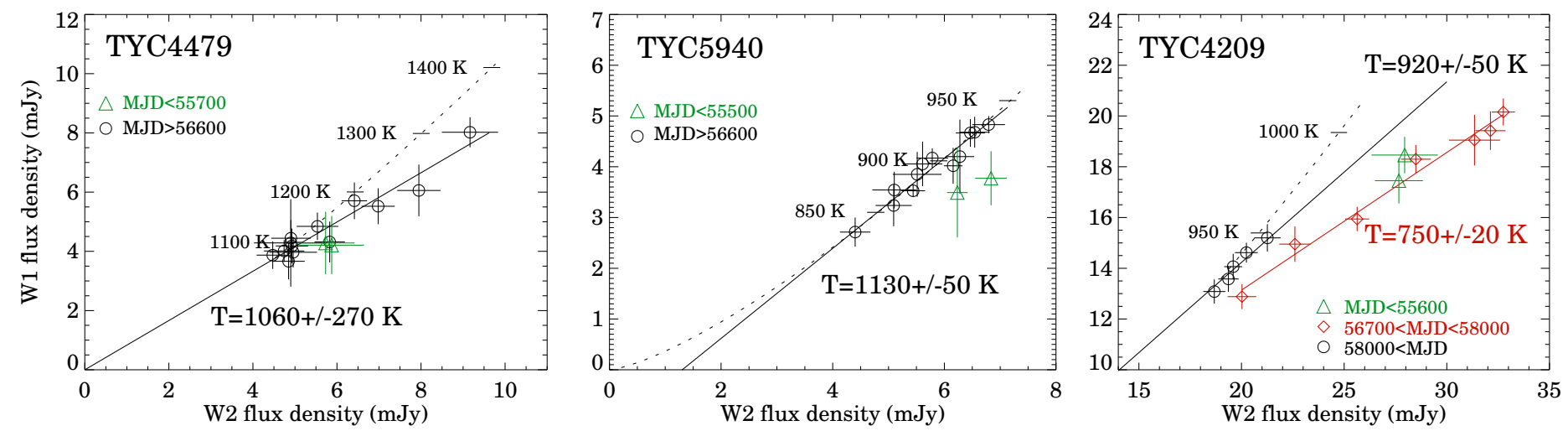

Figure 9. Correlation of the WISE $3.4 \mu \mathrm{m}$ and $4.6 \mu \mathrm{m}$ flux densities using the seasonal averages of three EDD formally exhibiting variability (Sect. 5, Fig. 7). Solid lines represent models of constant temperature with varying dust emitting surface, while dotted lines denote the emission of a dust population of increasing temperature with constant emitting surface.

inconsistent with a model where the temperature of the dust grains would increase above $1060 \mathrm{~K}$, because it would have made the resulting emission bluer which is not seen in the data (dotted curve). Thus, for the observed variability of TYC 4479, the most plausible model is the change of the effective surface area. It may be caused by production of fresh dust, but it may also be due to geometrical rearrangement of the ring structure, which would temporarily decrease the dust column density and make the IR emission less optically thick. The minimum emitting area in the faintest state (assuming optically thin blackbody radiation) was $0.00023 \mathrm{au}^{2}$, while at the brightness peak it rose to $0.00046 \mathrm{au}^{2}$, doubling the dust surface within a few months. Note that if the dust is partly optically thick then these estimates are lower limits. Taking into account the luminosity of the star (Tab. 1), the blackbody radius of the $1060 \mathrm{~K}$ dust is $\approx 0.06 \mathrm{au}$.

The infrared time variability of TYC 5940 (Fig. 9, middle) can be explained by both of our simple models. The data points form a straight line, which, however, does not reach the origin. The non-zero intercept is significant at the $5 \sigma$ level. The data are also compatible within the measurement uncertainties with a model of increasing dust temperature (dotted curve). Thus, one possible interpretation is that we see a dust population, located at $0.06 \mathrm{au}$ from the star at an equilibrium temperature of $1130 \mathrm{~K}$, which changed its emitting area between $0.00034 \mathrm{au}^{2}$ and $0.0006 \mathrm{au}^{2}$ over the observed period. The non-zero intercept may point to the existence of a colder dust population (probably at larger stellocentric radius), whose thermal emission at $3.4 \mu \mathrm{m}$ is still negligible, but at $4.6 \mu \mathrm{m}$ becomes detectable. An equally plausible model is, however, that the area of the dust ring remained constant while its temperature increased from $\approx 850 \mathrm{~K}$ to $\approx 950 \mathrm{~K}$. The origin of the energy needed for the extra heating is unlikely to arrive from the star (in Sect. 5.2 we argued about a low level of stellar variability), raising a question about the heat source.

The most complex case of variability is TYC 4209 . This source exhibited a broadly symmetric brightness peak in 2014-17 (Sect. 5), followed by smaller scale variations. The two periods show different color temperature behaviors (Fig. 7). In order to examine the physical reasons of variability separately, we plotted in Fig. 9 (right) the data points of the two periods with different symbols. The flux changes after the brightness peak (MJD $>58000$, black circle) can be modeled with a straight line reaching the origin within the uncertainties of the fit parameters. It would imply an increasing emitting surface of a $\mathrm{T}=920 \mathrm{~K}$ dust population, located at $0.11 \mathrm{au}$ from the star, from $0.0041 \mathrm{au}^{2}$ to $0.0047 \mathrm{au}^{2}$. The observations are also marginally consistent with the warming up of this dust ring from $\approx 920 \mathrm{~K}$ to $\approx 950 \mathrm{~K}$. The 2014-17 brightness maximum, however, seems to be intrinsically different from the previous process. It is clearly inconsistent with any variable dust temperature model. Since its emission appears as an addition to the flux of the $920 \mathrm{~K}$ dust ring, we suggest that we witness the appearance and subsequent disappearance of a cloud of fresh dust at a temperature of $\mathrm{T} \approx 750 \mathrm{~K}$, with an emitting area of $0.006 \mathrm{au}^{2}$. This temperature corresponds to a radius of $0.17 \mathrm{au}$, significantly farther than the first ring. Outside the most opaque core of the $\mathrm{T}=920 \mathrm{~K}$ ring the optical depth of the circumstellar dust is probably below unity, thus, a geometrical rearrangement of the dust at $\mathrm{r}=0.17$ au would be an unlikely cause of the emission peak, leaving the formation of new dust via collisions as the most likely physical picture.

The last, formally variable object, TYC 8105 could not be analysed with these techniques, because of the large 
error bars and the limited dynamic range of the data points.

The temperatures yielded above are higher than the characteristic dust temperatures derived from the blackbody fit to the $3.4-22 \mu \mathrm{m}$ band IR excess (Sect. 4.3). It may indicate that these disks present a range of dust temperatures and their dust material is distributed over a broad radial range.

\subsection{The nature of the EDD phenomenon}

In addition to the 6 systems studied in this paper, 11 additional EDDs are claimed in the literature (Appendix C). Apart from HD 113766 and HD 145263, where the host stars are F2-type dwarfs, all these disks surround solar-type (F5-K3 type) stars. The nature of two of these additional disks is still debated. RZ Psc was found to exhibit a weak accretion signature, suggesting that the disk may be of primordial nature rather than EDD (Potravnov et al. 2017, 2019). Concerning HD 166191, Kennedy \& Wyatt (2014) raised the possibility that it is not a secondary disk but a primordial transition disk. However, based on recent millimeter continuum and line observations of this system, both the measured low CO gas mass and the low gas-to-dust mass ratio are more consistent with a debris disk nature (Garcia \& Hughes 2019). Despite their debated classification, both objects are included in our analysis. The case of TYC 8241-2652-1 is quite special: before 2010 this young K-type star hosted a warm $(\sim 450 \mathrm{~K})$, unusually dust-rich disk, whose mid-IR luminosity then decayed significantly over less than two years, leaving behind a colder $(<200 \mathrm{~K})$, tenuous disk (Melis et al. 2012). Recent observations indicated that the disk has remained depleted (Günther et al. 2017). Table C1 shows the parameters valid before 2009, which were consistent with an EDD. In the following, we will consider the whole sample of EDDs, including all sources, proposed in the literature and the ones in this paper, to examine their mid-infrared variability, age distribution, and the multiplicity of the host stars. We will also assess what these characteristics indicate about the nature of EDDs.

\subsubsection{Mid-infrared variability of EDDs}

Many of the previously known 11 EDDs were targets of multi-year photometric monitoring campaigns performed by Spitzer at $3.6 \mu \mathrm{m}$ and $4.5 \mu \mathrm{m}$. These studies revealed significant variability on monthly to yearly timescales - in some cases with very complex variability patterns - in seven of them: HD 15407, HD 23514, BD+20 307, ID 8, P 1121, HD 113766, and HD 166191 (Meng et al. 2014, 2015; Su et al. 2019b,a, 2020). HD 145263 was found to show no significant disk variations based on its Spitzer observations in 2013 (Meng et al. 2015).

In addition to Spitzer data, WISE measurements are available for all 11 sources allowing us to study their mid-IR flux changes over the AllWISE and NEOWISE Reactivation mission phases between 2010 and 2019. The steps and results of this analysis are presented in Appendix C. The three brightest sources, HD 15407, HD 113766, and HD 166191, are saturated in both bands, preventing us from drawing reliable conclusions regarding their variability (we note that their Spitzer data point to substantial variability). A similar issue affects the W1 photometry of HD 145263 and $\mathrm{BD}+20307$. The W2 light curves of the latter two objects displayed no significant variability over the given period.

As Figure C1 demonstrates, for HD 23514, ID 8, and P 1121, WISE measurements confirm the previous Spitzer-based results, proving that these disks exhibit well detected flux changes between 3 and $5 \mu \mathrm{m}$. While during the Spitzer observations in 2013, the disk of HD 23514 showed a fading trend, its NEOWISE Reactivation measurements indicate a slow brightening since 2014. For ID 8, WISE measurements from 2018-2019 complement the previous Spitzer data, showing that the disk continues to display significant changes. The WISE light curves of RZ Psc (see also in Kennedy et al. 2017) and V488 Per show evidences of strong variability in both the W1 and W2 bands. It is noteworthy that between January and August 2019, the disk of V488 Per became $\sim 7$ and $\sim 4$ times brighter at 3.6 and $4.5 \mu \mathrm{m}$, respectively, and it is now brighter at these wavelengths than ever observed before. This is the largest increase we have seen in any EDDs at any given six month interval. Although the WISE fluxes of TYC 8241-2652-1 did not show significant changes between 2010 and 2019, before this period, its disk underwent a dramatic fading (Melis et al. 2012).

Thus, in the case of the previously known EDDs there were significant flux changes in 10 out of the 11 sources, while in our sample 4 disks out of the 6 proved to be variable. In summary, 14 out of the 17 EDDs have shown significant variability in the wavelength range of 3 to $5 \mu \mathrm{m}$ over the past roughly ten years. Flux changes can be observed even in the oldest EDD systems, both TYC 4209 and TYC 4479 exhibit substantial variations. These results indicate that the variability phenomenon is an inherent characteristic of EDDs.

Although the light curves of EDDs show a large variety in their shape, some of them exhibit similar patterns. Between 2015 and 2019, the disks of HD 23514 (Fig. C1) and TYC 5940 (Fig. 7) showed analogous slowly rising 
trends both at 3.4 and $4.6 \mu \mathrm{m}$. The light curves of ID 8 display a wavy pattern (Fig. C1), the successive brightening and fading periods occur on a yearly timescale and probably represent the aftermath of violent impact events (Su et al. 2019b). As Fig. 7 demonstrates, the WISE data of TYC 4209 and TYC 4479 show similar ripples, although in these cases, in contrast to ID 8, the rising phase is steeper than the declining one. These bumps might also be attributed to significant dust releasing events. In the case of ID 8, Su et al. (2019b) found that the observed $4.5 \mu \mathrm{m}$ flux change associated with the decay in 2013 corresponds to a total cross section change of $0.0021 \mathrm{au}^{2}$. Based on WISE data, the inferred cross section change at TYC 4209 is $\sim 3 \times$ higher, while at TYC 4479 , it is $\sim 9 \times$ lower (Sect. 6.2).

\subsubsection{Wide separation companions}

Examining a smaller EDD sample available at that time (five disks around 30-100 Myr old solar-type stars), Zuckerman (2015) suggested that these disks are situated preferentially in wide binaries. Considering that five of our disks are also found in similar systems, it is worth looking again at this possible relationship using the now much larger sample. As it is mentioned above, out of the previously known 11 EDDs, 9 have solar-type (F5-K3 type) host stars, from which so far five are proven multiples. $\mathrm{BD}+20307$ is composed of two late F-type main-sequence stars in a close orbit (P 3.4 day, Weinberger 2008; Zuckerman et al. 2008). Using GaiaDR2 astrometric data, Hartman \& Lépine (2020) identified a new common proper motion companion of this star at a projected separation of 8.'4 (980 au). This wide companion is classified as a white dwarf candidate with a mass of $0.48-0.58 \mathrm{M}_{\odot}$ (depending on whether a pure-He or pure-H atmosphere is supposed, Gentile Fusillo et al. 2019). Recently, Kennedy et al. (2020) reported the discovery of a low mass companion of RZPsc at a projected separation of $23 \mathrm{au}$. The other three stars, HD 15407, HD 23514, and V488 Per, are all reported to have wide-orbit companions, the projected separations are $\rho_{\mathrm{p}}=1050,365$, and $12300 \mathrm{au}$, respectively (Melis et al. 2010; Rodriguez et al. 2012; Zuckerman 2015). V488 Per possibly hosts a second lowmass companion at $\rho_{\mathrm{p}} \sim 12000$ au (Zuckerman 2015).

Using the method and requirements we described in Sect. 3.3, we searched the Gaia EDR3 catalog for previously unknown common proper motion pairs of these nine stars. This confirmed the findings of Hartman \& Lépine (2020) for BD+20307 and yielded new faint candidate pairs for three more stars, V488 Per (five candidates, all with $\rho_{\mathrm{p}}>0.39 \mathrm{pc}$ ), HD 23514 (one candidate, $\rho_{\mathrm{p}}=0.41 \mathrm{pc}$ ), and $\mathrm{P} 1121$ (one candidate, $\left.\rho_{\mathrm{p}}=0.74 \mathrm{pc}\right)$. However, all of these stars are members of young open clusters (V488 Per: $\alpha$ Per open cluster, HD 23514: Pleiades, P 1121: NGC 2422). By studying wide binary systems in the $\alpha$ Per, Pleiades, and Praesepe open clusters, Deacon \& Kraus (2020) demonstrated that at projected separations $>3000$ au it is difficult to separate true binaries and unrelated cluster members. Although it cannot be completely ruled out that some of the revealed candidates are true binaries, the separations suggest that they instead fall into the latter category. Therefore, these were not taken into account in our further analysis. Based on the same arguments, Deacon \& Kraus (2020) do not list V488 Per as a probable wide binary, because, due to their large separations, the previously reported companions (at $\rho_{\mathrm{p}} \sim 12000 \mathrm{au}$, see above) could also be unrelated cluster members. Therefore, in the following, we do not consider this system as a justified binary.

Putting it all together with our results, out of the 15 EDDs with solar-type hosts, there are 8 systems $\left(53.3_{-12.4}^{+11.6} \%\right)$ with wide separation $(365-6010 \mathrm{au})$ companions. Remarkably, all of them are older than $100 \mathrm{Myr}$. Thus, at ages <100 Myr (5 EDDs out of the 15) no wide binaries were found, while at ages $>100 \mathrm{Myr}$ (10 EDDs), the multiplicity fraction is $80_{-17.2}^{+7.1} \%$. Considering the small sample, the uncertainties were derived by adopting a binomial distribution (Burgasser et al. 2003). For the majority of the EDD systems the information about wide companions is based on data from the Gaia EDR3 catalog. There are possible limitations of this approach. By testing the small-scale completeness of this data release based on source-pair distances in a small dense field near the Galactic plane, Fabricius et al. (2020) claimed that the completeness falls rapidly at separations less than 0.7 . This value corresponds to $\sim 300$ au at P 1121 , which is the farthest object in the sample. Of course the detection sensitivity of possible companions depends not only on the angular separation but on the brightness ratio of the pair as well (Brandeker \& Cataldi 2019). Even in pairs with large angular separations, it is possible that the companion is too faint to have a proper astrometric solution in the current Gaia data release. Future observations with ground-based high-contrast facilities and with Gaia, have the potential to reveal additional fainter and less wide companions in these systems.

In order to put these results into context, we need to know the incidence of similar binaries among solartype stars. Based on the log-normal distribution derived by Raghavan et al. (2010) for separations of companions around F6-K3 type main-sequence stars, about $13 \%$ of such dwarfs have at least one companion at separations 
between 300 au and $1 \mathrm{pc}$. This fraction is $\sim 4 \times$ lower than what we observe among solar-type EDD stars. By applying a binomial test (using the $\mathrm{R}$ statistical programming language's binom. test function) we obtain a probability of $2.3 \times 10^{-4}$ for the null hypothesis that the fraction of wide binaries in the EDD sample is equal to or lower than in the comparison sample. If we consider only objects older than $100 \mathrm{Myr}$ from the EDD sample, the contrast is even stronger, the observed wide companion fraction in that subsample is $\sim 6 \times$ higher than among normal solar-type stars. In this case, a binomial test yields a probability of $3 \times 10^{-6}$ for the abovementioned null hypothesis, i.e. it could be rejected with a high statistical reliability.

However, while most EDD host stars are likely younger than $300 \mathrm{Myr}$, the comparison sample predominantly contains older stars. This must be taken into account in the comparison, since the frequency of weakly bound wide binaries can decline with time as a result of gravitational influence due to encounters with other stars and giant molecular clouds (Weinberg et al. 1987). In a recent survey of the $10 \mathrm{Myr}$ old Upper Scorpius association, Tokovinin \& Briceño (2020) found that the fraction of $100-10^{4}$ au pairs with solar type primaries resembles that of stars in the field. By scrutinizing members of nearby young moving groups (5-100 Myr old), Elliott et al. (2015) also claimed that the multiplicity frequency of solar type stars in the separation range of $10-1000$ au is similar to that in the field star sample compiled by Raghavan et al. (2010). Deacon \& Kraus (2020) confirmed these results by reaching the conclusion that the fraction of pairs with separations of $>300$ au among FGK-type stars belonging to nearby 10$200 \mathrm{Myr}$ old young moving groups and to the $\sim 125 \mathrm{Myr}$ old Pisces-Eridanus stream shows no significant excess over that of similar type older field stars. In the same study, they showed that compared to these low density formation environments and field stars, there appears to be a deficit of wide (300-3000 au) binaries in open clusters. Actually, the $\alpha$ Per, Pleiades, and Praesepe clusters have an average binary fraction of $3 \%$ (with FGK-type primaries) in this projected separation range. We note that in the 'old' (>100 Myr) EDD sample the corresponding fraction is $6 / 10$. Based on these results, there is no indication that the incidence of wide binaries would be significantly higher in younger samples confirming that our previous comparisons with the field star sample do not require corrections. Our results thus imply that very wide-orbit pairs are more common in EDD systems than in the normal stellar population.

\subsubsection{Age distribution and disk evolution}

EDDs are thought to be produced in violent collisions occuring between planetary embryos during the final accumulation of terrestrial planets (Jackson \& Wyatt 2012; Genda et al. 2015; Su et al. 2019b). If so, then by studying their age distribution, we can put observational constraints on the timeline of rocky planet formation around Sun-like main-sequence stars. Figure 10 shows the ratios of the measured 4.5 or $4.6 \mu \mathrm{m}$ flux densities to the predicted stellar photospheric fluxes as a function of ages for all 17 EDDs. Variability flux ranges, when applicable, are shown by vertical dotted lines. For BD+20307, HD 15407, and HD 113766, we used Spitzer $4.5 \mu \mathrm{m}$ photometry taken from the literature (Meng et al. 2015; Su et al. 2019b). HD 166191 exhibited a strong brightening in 2019 (Su et al. 2019a), the corresponding individual Spitzer data points are from $\mathrm{Su}$ et al. (2021, in preparation). For the rest of the sample WISE $4.6 \mu \mathrm{m}$ data are plotted, where the minimum and maximum fluxes were taken from the light curves plotted in Figs. 7 and C1. In the case of TYC 8241-2652-1, only the minimum flux was obtained in this way, the maximum flux at $4.6 \mu \mathrm{m}$ was estimated from its disk model constructed by Melis et al. (2012) considering the IRAS measurements of the source. For HD 145263, which is constant based on both Spitzer and WISE time-domain data, AllWISE W2 band photometry was utilized, after correcting for saturation by applying the method described in Cotten \& Song (2016)

In our solar system, the era of giant impacts probably ended by a collision between the proto-Earth and another body that led to the formation of the EarthMoon system (Canup \& Asphaug 2001). Analyses of radioactive isotopes yield age estimates between 30 and $110 \mathrm{Myr}$ for this event (Jacobsen 2005; Touboul et al. 2007; Halliday 2008; Kleine et al. 2009). Quintana et al. (2016) used $N$-body simulations to examine the formation of planets in the terrestrial zone of a Sun-like star that harbors giant planets analogous to Jupiter and Saturn. Their initial disk included 26 Mars-sized planetary embryos and 260 smaller, approximately Moonsized bodies. They found that most of the collisions took place in less than $100 \mathrm{Myr}$ : in models where fragmentations were enabled, $90 \%$ of collisions occurred within the first $82 \mathrm{Myr}$, and $50 \%$ of them in the first $20 \mathrm{Myr}$. Nevertheless, based on the simulations, large collisions can happen even after hundreds of millions of years (up to $1 \mathrm{Gyr}$ ), although these are quite rare. Genda et al. (2015) reached a similar conclusion in their simulations without giant planets: the final giant impacts were found to occur at $73 \pm 74 \mathrm{Myr}$. The age distribution of EDDs differs significantly from these results. Although most of the systems are probably younger than $500 \mathrm{Myr}$, 


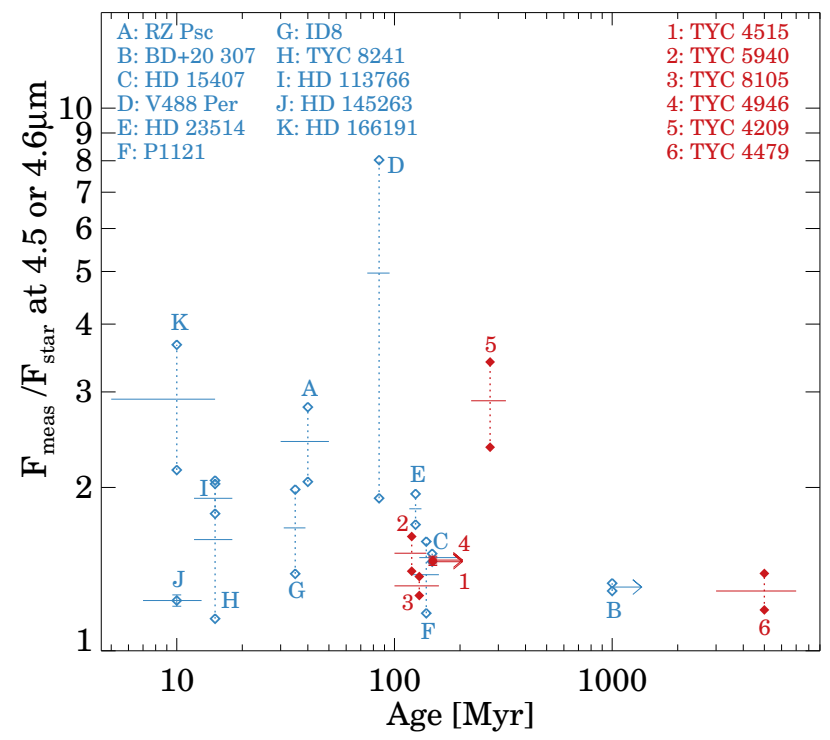

Figure 10. Ratios of the measured WISE2 band $(4.6 \mu \mathrm{m})$ flux densities to stellar photospheric fluxes as a function of age for extreme debris disks. Blue empty symbols show the eleven previously known objects, while red filled symbols denote our targets from Table 1. Variability ranges are shown by vertical dotted lines.

and thus can even be the result of a similar major collision, the majority of them ( $\sim 60 \%$ of the sample) are older than $100 \mathrm{Myr}$, and at least 3 of them $(\sim 20 \%)$ have an age of $>200 \mathrm{Myr}$. Thus if EDDs are associated with rocky planet formation, then our results displayed in Fig. 10 hint that the intensity of these processes - contrary to what solar system experienced and what is predicted by the simulations - does not decay significantly even after $100 \mathrm{Myr}$. Obviously, the simulations examined only a fraction of the possible parameter space. The presence of giant planets and their architecture, as well as the mass and location of embryos/planetesimals in the terrestrial zone at the beginning of the giant impact era may have a serious influence on the timeline of planet formation (Quintana et al. 2016; Barclay et al. 2017). However, it is questionable whether all these can cause such prolongation of rocky planet formation processes as suggested by our results.

\subsubsection{Alternative scenarios for the formation of EDDs}

Alternatively, it is possible that some other mechanism(s) can also produce EDDs and this process is more typical in slightly older systems. When speculating on such mechanisms, we must consider that, based on the available observational characteristics, the older EDDs are quite similar to the younger ones. Though the average fractional luminosity of disks older than $100 \mathrm{Myr}$ is somewhat lower than that of the younger objects, their characteristic dust temperatures are similar and they display mid-IR variations with the same frequency and comparable amplitudes. A possible alternative mechanism should be able to explain these properties.

Single giant collision in an inner planetesimal belt-Although the formation of EDDs is certainly not explicable by the long term steady state collisional evolution of an in situ planetesimal belt (Sect. 6.1), individual giant impacts may happen in a collisionally depleted belt as well. However, considering that during the collisional evolution the number of large bodies, whose destruction can reproduce the observed dust quantity, is also decreasing, the probability of witnessing such an event falls off as $\propto t_{\text {age }}^{-2}$ (Wyatt et al. 2007). This is inconsistent with the observed age distribution. Actually, according to Wyatt et al. (2007), disks with high $f_{\mathrm{d}} / f_{\mathrm{d}, \max }$ (Sect. 6.1), which cannot be the result of steady state evolution of a planetesimal belt, cannot be explained with such single giant collisions either.

Sublimation and distruption cometary bodies-Comets transported from an outer reservoir into the inner regions are also widely considered as possible sources of warm debris material (e.g., Wyatt et al. 2007; Morales et al. 2011; Bonsor et al. 2012; Ballering et al. 2017; Marino et al. 2017). Actually, in our solar system the zodiacal dust particles dominantly stem from comets (Nesvorný et al. 2010; Rowan-Robinson \& May 2013). Detections of non-photospheric, sometimes variable, absorption features toward several stars imply cometary activity in other systems as well (e.g., Beust et al. 1991; Kiefer et al. 2014; Welsh \& Montgomery 2015; Iglesias et al. 2018; Rebollido et al. 2020). Due to the subtantially longer collisional times, the depletion of cold outer planetesimal belts is much slower than that of inner Asteroid Belt analogs. Therefore, minor bodies originating from such reservoirs can supply dust in the inner zones even long after the local belt has largely been exhausted. These icy bodies can contribute to the production of warm dust in several ways. Dust grains can arise from the sublimation and disruption of comets, as well as from collisions of the inwardly scattered objects with each other and/or with inner rocky bodies.

Planetesimals can evolve into more eccentric orbits under the gravitational influence of planets or stellar companions, or because of a close stellar encounter. Investigation of scattering processes revealed that efficient, long term inward transport of planetesimals from an outer belt requires a special architecture: the presence of a chain of closely spaced low mass planets (Bonsor et al. 2012, 2014; Marino et al. 2018). As Faramaz et al. 
(2017) demonstrated, interactions between planetesimals and an outer eccentric planet can also drive a vast number of icy bodies to highly eccentric orbits, which then can produce warm dust grains in the inner system on gigayear time scales. Transient events with a shorter time scale may also be of interest. A rearrangement of the planetary system following a dynamical instability akin to the hypothesized Late Heavy Bombardment scenario in our solar system (Tera et al. 1974; Booth et al. 2009; Bottke \& Norman 2017) - can cause shorter lived, significantly enhanced cometary activity. A companion or a star passing close-by might also result in a transient spike in the warm dust production by initiating a comet shower from an outer planetesimal belt or from a reservoir analogous to the solar system's Oort cloud.

In the following, we examine whether the cometary scenario can explain the peculiarly high dust content of EDDs. As a minimal EDD, we consider a $0.25 \mathrm{au}$ wide debris belt that is located at 0.5 au around a Sun analog $\operatorname{star}\left(L_{*}=1 L_{\odot}, M_{*}=1 M_{\odot}\right)$ and has a fractional luminosity of 0.01 . To estimate its mass loss rate, we used eq. 29 from Wyatt et al. (2007) and obtained $\sim 0.17 \mathrm{M}_{\oplus} / \mathrm{Myr}$. Supposing a dust to ice mass ratio of 1 in the comets and that their material is converted to dust grains with a $100 \%$ efficiency, the sustenance of the above model EDD over $100 \mathrm{Myr}$ would require the destruction of $34 \mathrm{M}_{\oplus}$ of icy planetesimals in the inner zones. By simulating various multiplanet architectures, Marino et al. (2018) found that at most $\sim 7 \%$ of planetesimals scattered from an outer belt via the planets reached the inner regions. Taking this maximum fraction, the long term supply of the disk in a steady state manner would require an unrealistically high total planetesimal mass of $\gtrsim 500 \mathrm{M}_{\oplus}$ in the outer reservoir. This suggests that even in the cometary scenario, it is more likely that the observed phenomenon is related to a short-lived transient event rather than a continuous long term comet flux.

Based on the cometary model developed by Marboeuf et al. (2016), we can make an estimate of how many active comets would be needed to provide the above derived dust production rate. Using their eq. 1719 , we find that a comet with a radius of $1 \mathrm{~km}$ located 0.5 au from a star with $L_{*}=1 L_{\odot}$ releases dust with a rate of $\sim 5300 \mathrm{~kg} \mathrm{~s}^{-1}$. Roughly 6 million such active comets are needed to supply our minimum EDD. Supposing larger icy planetesimals with a radius of $100 \mathrm{~km}$, i.e., with a size roughly corresponding to the largest Centaurs in the solar system, the required number would be $\sim 600$. Since a large fraction of scattered icy bodies never reaches the snow line and even those that do, spend only a part of their orbital time within the line, the total number of comets involved in the process has to be much higher than this. However, this calculation only considers the dust production driven by the sublimation of icy bodies. According to Nesvorný et al. (2010) in the Solar system the spontaneous disruption of Jupiter family comets (JFCs) is the main source of the zodiacal dust. Moreover, the presence of a large number of comets in the terrestrial zone increases the chance of collisions with inner planets and other comets. Thus, it is entirely possible that in EDDs the latter two processes dominate the supply of dust and, therefore, much fewer active comets are sufficient to explain the observed excess.

Disruption of large comets and giant impacts is accompanied by rapid, vigorous dust release that can also explain the high amplitude mid-IR changes seen in EDDs. For TYC 4479 and TYC 4209, the observed midIR brightening requires a minimum dust cross section change of $2.3 \times 10^{-4} \mathrm{au}^{2}$ and $6.0 \times 10^{-3} \mathrm{au}^{2}$, respectively (Sect. 6.2). How large should a disintegrating comet be to produce the necessary amount of dust? In the solar system, disruption of JFCs produces mm to cm-sized particles that later collisionally grind down to smaller fragments (Nesvorný et al. 2010). Assuming that the dust material of the comet (half of its total mass) turns into $0.5 \mu \mathrm{m}-1 \mathrm{~mm}$ grains in a power-law size distribution with an index of -3.5 and taking an dust/ice density ratio of 3 , the reproduction of the derived cross sections needs the disruption of a planetesimal with a radius of $\sim 50 \mathrm{~km}$ and $\sim 160 \mathrm{~km}$ for TYC 4479 and TYC 4209, respectively. These sizes should be considered as lower limits since 1) the disruption may not be so efficient; 2 ) the average size of the emerging particles is probably underestimated; and 3) the estimated cross section values are also lower limits (Sect. 6.2). We note that collisions between objects of the sizes computed above can also provide a good explanation for the observed flux changes (e.g., Su et al. 2019b).

The possible role of dynamical instabilities - As mentioned earlier, the temporarily enhanced comet activity could be due to a dynamical instability of the planetary system. On their changed orbit, planets can perturb neighboring planetesimal populations, leading to their excited eccentricities, causing an increased flux of infalling icy planetesimals in the terrestrial regions. Although the aftermath of such instabilities can last for up to a few $10 \mathrm{Myr}$, the enhanced level of exozodiacal dust typically decays on a time scale of a few million years (Bonsor et al. 2013). Figure 2 of Bonsor et al. (2013) suggests that even within this period, there could be a shorter peak in the comet flux. By the end of such an instability event, the outer planetesimal belt can be 
substantially depleted (Booth et al. 2009). The mass inflow of comets might be sufficiently high to produce an extreme debris disk. However, although the NICE model of the solar system demonstrates that a dynamical instability can be delayed to hundred million years (Gomes et al. 2005), Bonsor et al. (2013) claims that such instabilities are more frequent in younger systems (typically $<10 \mathrm{Myr}$ ). Thus, this mechanism, similarly to the rocky planet formation scenario, is unable to offer a solution for the observed age distribution, although it may explain the origin of some individual systems.

The possible influence of wide companions - Intriguingly, we found that very wide-orbit pairs are more common in EDD systems than in the normal stellar population (Sect. 6.3.2). This is particularly true for EDDs older than 100 Myr: out of the 10 objects, 8 are located in wide binaries. This raises the question whether the two phenomena may be physically connected. One possibility is that, as a consequence of impulses from passing stars and torques from the Galactic tide, orbits of very wide ( $a \gtrsim 1000 \mathrm{au}$ ) binary stars can become very eccentric after a time (Jiang \& Tremaine 2010; Kaib et al. 2013; Bonsor \& Veras 2015). Due to the decreasing pericenter radius, the companion can launch a comet shower when approaching the outer population of planetesimals. Indeed, the 365-6000 au projected separations found in the sample are comparable to the size of the scattered disk or the Oort cloud in the solar system. This mechanism is able to produce an excess population of long period comets. A favorable aspect of this model is that the orbital change of the companion requires time, thus this process could occur much later than $100 \mathrm{Myr}$, too. However, this hypothesis has several drawbacks. Long period comets are on very eccentric orbits and spend only a small fraction of their revolution within the snow line thus the dust production is limited. Moreover, simulations of perturbations caused by a companion or a stellar flyby predict a comet flux increase of at most a few orders of magnitude, which means a few tens of such comets per year (Davis et al. 1984; Berski \& Dybczyński 2016), far below the required level. Therefore, this scenario alone probably cannot explain the observed dust contents.

A companion with an initial inclination $\left(i_{\mathrm{c}}\right)$ between $\sim 39^{\circ}$ and $\sim 141^{\circ}$ with respect to the orbital plane of a third body (e.g. a planetesimal or a planet) can cause oscillations in the eccentricity and inclination of this body via the Kozai-Lidov (KL) mechanism (Naoz 2016). The timescale of this effect depends on the mass $\left(m_{\mathrm{c}}\right)$, orbital period $\left(P_{\mathrm{c}}\right)$, and eccentricity $\left(e_{\mathrm{c}}\right)$ of the companion, the total mass of the primary, the perturber, and the disk $\left(m_{\mathrm{tot}}=m_{\mathrm{pr}}+m_{\mathrm{c}}+m_{\mathrm{disk}}\right)$, and the orbital period of the disk's particles $(P)$ (Nesvold et al. 2016):

$$
t_{\mathrm{KL}} \sim \frac{m_{\mathrm{tot}}}{m_{\mathrm{c}}} \frac{P_{\mathrm{c}}^{2}}{P}\left(1-e_{\mathrm{c}}^{2}\right)^{3 / 2} .
$$

The first eccentricity peak is achieved at $t_{\mathrm{KL}} / 2$, the maximum of the eccentricity can be approximated as $\sqrt{1-(5 / 3) \cos ^{2} i_{\mathrm{c}}}$ (Naoz 2016; Nesvold et al. 2016). Assuming that the detected wide companions fulfill the inclination criteria above $\left(39^{\circ} \leq i_{c} \leq 141^{\circ}\right)$ and are on a circular orbit, and adopting the projected separations as an estimate of the semi-major axes we can calculate the innermost radius where a third body can undergo the maximum eccentricity phase during the lifetime of system. In the cases of TYC 4209 and TYC 8105 , we deduced radii of $\sim 140 \mathrm{au}$ and $\sim 240 \mathrm{au}$, respectively. Even the largest known exo-Kuiper belts around solar-type stars have radii $<200$ au (Löhne et al. 2012; Faramaz et al. 2019; Sepulveda et al. 2019). Considering this, and that the eccentricities already started increasing inside the above derived radii, it is conceivable that in TYC 4209 the companion can affect the orbit of planetesimals in an outer belt. A direct entry of a perturbed planetesimal into the sublimation region inside the snow line is possible but would require a high eccentricity (e.g. $e>0.99$ for a comet originating from a belt at $>100 \mathrm{au}$ ), and thus would require a high inclination of the companion $\left(i_{\mathrm{c}} \gtrsim 84^{\circ}\right.$ for the previous example). However, even with smaller eccentricities, a planetesimal can cross a chaotic zone of a planet from where it can be transported inward, potentially by multiple scattering in a chain of planets. In BD+20 307, HD 23514, HD 15407, TYC 4479, TYC 4946, and TYC 4515, the eccentricity of a tertiary body can peak within the available time (the age of the system) already at $<1.5 \mathrm{au}, \sim 2 \mathrm{au}, 4 \mathrm{au}, 11 \mathrm{au},<8 \mathrm{au}$, and $<15$ au, respectively, due to the K-L mechanism. It means that in these cases, not only the outer planetesimal belt could be affected, but also the possible planetary system within the belt. Changing eccentricities of the planets can lead to a dynamical instability that can enhance the inward transport of comets further (see above). It is also a delayed effect, which can be consistent with the existence of EDDs at $>100 \mathrm{Myr}$.

In this calculation, for the probable white dwarf companion of $\mathrm{BD}+20307$ we adopted a mass of $0.48 \mathrm{M}_{\odot}$ (Gentile Fusillo et al. 2019) and a semi-major axis of $980 \mathrm{au}$. However, the progenitor of this companion was more massive and the mass loss during the stellar evolution was accompanied by a change in its orbit, making our result on the K-L mechanism in this system uncertain. Further investigation of the white dwarf companion would be important to provide a more accurate age 
determination (e.g. Fouesneau et al. 2019) and to study the dynamical evolution of this interesting triple system. This could lead to a better understanding of the formation of the dusty warm disk as well.

For the semi-major axes of the companions, we adopted the observed projected separations, which are likely smaller than the true semi-major axes. Considering a random distribution of orbital inclinations and the observed eccentricity distribution of binary systems Fischer \& Marcy (1992) found that the true semi-major axes are $1.26 \times$ larger on average than the projected separations. Taking into account this multiplicative factor in a statistical manner results in $\sim 1.6 \times$ larger inner radii for the area affected by the K-L mechanism. This would not change our main conclusions.

Most cometary models assume the presence of an outer, cold planetesimal belt in the system. Based on currently available observations, none of the EDDs with age $>100$ Myr show evidence for cold debris belts. Three systems have deep far-infrared photometry obtained with the Herschel Space Observatory. For HD 15407 and HD 23514, these observations implied the presence of two temperature components, however, even the colder dust has relatively high temperatures; $334 \mathrm{~K}$ and $168 \mathrm{~K}$, respectively (Vican et al. 2016). These temperatures are higher than what is required for sublimation of water ice, and thus they do not indicate regions in which icy planetesimals can persist for a long time. Considering their fractional luminosity, these components, even if they indicated separate rings, could still be of transient origin (Vican et al. 2016). In the case of BD+20 307, the 70 and $100 \mu \mathrm{m}$ Herschel photometries are consistent with the emission of a single warm component. P 1121 harbors no cold dust either (Meng et al. 2015; Su et al. 2019b). As for our six objects, the IRAS FSC catalog provides upper limits at $60 \mu \mathrm{m}$ for four of them (TYC 4515, TYC 5940, TYC 8105, and TYC 4209). For the other two targets, we used the IRAS SCANPI tool ${ }^{14}$ to derive upper limits in the same band. On the basis of these IRAS data, even in the best cases, it could only be established that there are no cold $(30-80 \mathrm{~K})$ disks with a fractional luminosity greater than 0.01 in our systems. Though there is no direct evidence for colder belts, in most cases the current data cannot exclude the existence of massive outer debris disks.

It is worth noting that in our previous analyses, we tacitly assumed that only grains for which the ratio of radiation pressure to gravitational force, $\beta$, exceeds 0.5 are blown out from the system $\left(\beta_{\mathrm{bl}}=0.5\right)$,

\footnotetext{
14 https://irsa.ipac.caltech.edu/applications/Scanpi/
}

which is valid if the parent bodies are on a circular orbit. Considering parent bodies on eccentric orbits $\left(e_{\mathrm{pb}}>0\right)$, such as comets, the $\beta_{\mathrm{bl}}$ can be computed as $\beta_{\mathrm{bl}}=0.5\left(\frac{1-e_{\mathrm{pb}}^{2}}{1+e_{\mathrm{pb}} \cos \Phi}\right)$, where $\Phi$ is the longitude of the release position on the orbit (Murray \& Dermott 1999; Sezestre et al. 2019). This means that depending on the eccentricity, particles with much smaller $\beta$ parameters (with much larger sizes) can become unbound and thus the released dust is removed more rapidly than in the circular case. Given typical comet orbits, this obviously requires higher replenishment rates than what obtained from our calculations.

\section{SUMMARY}

In this study, we conducted a survey to search for EDDs around Sun-like stars. Using the AllWISE infrared photometric and the Gaia TGAS astrometric catalogs, and applying careful selection criteria, we found six new EDDs: TYC 4515, TYC 5940, TYC 8105, TYC 4946, TYC 4209, and TYC 4479. Previously, only one of them, TYC 4479, was identified as a debris disk - but not as an EDD - in the literature (Cotten \& Song 2016). Host stars of these disks are main-sequence F5G9 stars located at distances between 164 and $279 \mathrm{pc}$ from the Sun. For their age estimates, we combined different empirical diagnostic methods based on the stars' lithium content, rotation, and kinematic properties. While the youngest objects have ages similar to that of the Pleiades $(\sim 120-130 \mathrm{Myr})$, the oldest one, TYC 4479 , has an age of $\sim 5 \mathrm{Gyr}$. Actually, the latter system is by far the oldest known EDD. Using astrometric data from Gaia EDR3, we found that five among the six host stars have co-moving pairs with projected separations ranging from 1820 to $6000 \mathrm{au}$. All these wide separation companions are low-mass, M-type stars.

To estimate the basic disk properties, we fitted a simple blackbody model to the observed IR excesses. The dust temperatures are higher than $300 \mathrm{~K}$ in all cases, and the derived fractional luminosities range between 0.01 and 0.07. Considering the ages, the observed high amount of warm dust indicates that these systems likely underwent a recent transient event of dust production. Using time-domain photometric data at 3.4 and $4.6 \mu \mathrm{m}$ from the WISE all sky surveys between 2010 and 2019, we concluded that the light curves of four systems (TYC 5940, TYC 8105, TYC 4209, and TYC 4479) show evidence of variable mid-IR emission on yearly timescales. We deduced that these variations are associated with the disks and not to the stars. By analyzing the observed 3.4 and $4.6 \mu \mathrm{m}$ variations, we found that at TYC 4209 and TYC 4479, the observed brightening events seem to be inconsistent with a model where 
the emitting area of the dust remains constant while its temperature increases. It is more likely that new dust was created temporarily. At TYC 4209, we discovered mid-IR variations at daily timescales as well.

With the six disks studied in this paper, the number of known EDDs increased to 17. Fifteen of them surround Sun-like (F5-K2) stars, while two have earlier F-type hosts. Using this new, unified sample we assessed what they indicate about the nature of these interesting objects. From the 15 Sun-like stars at least 9 reside in multiple systems, 8 of which are wide separation binaries with projected separations $>365$ au. By comparing this incidence with the corresponding statistics for solar-type field stars, we found that very wide-orbit pairs are significantly more common in EDD systems than in the normal stellar population. The contrast is even stronger if we consider only EDDs older than $100 \mathrm{Myr}$ (10 objects), since all 8 wide binary systems belong to this subsample. Based on the literature and on our analysis, 14 of the 17 known EDDs showed changes at $3-5 \mu \mathrm{m}$ over the past decade. This suggests that the mid-IR variability is an inherent characteristic of EDDs.

The formation of EDDs is generally thought to be linked to giant impacts occuring during the final, chaotic growth phase of terrestrial planets. In our solar system, this phase may have lasted up to $\sim 100$ million years. General numerical simulations of rocky planet formation predict that this era could extend up to a few hundred million years, but the vast majority of giant collisions happen in the first $100 \mathrm{Myr}$. The observed age distribution of the currently known EDDs is inconsistent with this picture. Only seven of them are younger than $100 \mathrm{Myr}$ and we know of at least two EDDs that are certainly older than $1 \mathrm{Gyr}$. If EDDs are indeed linked to rocky planet formation, then these results suggest that the intensity of this process does not decline significantly even after $100 \mathrm{Myr}$. Alternatively, some other mechanism(s) also produce EDDs. One possible explanation is that the observed dust comes from the disruption and/or collisions of comets delivered from an outer reservoir into the inner regions.

A high fraction of the oldest EDDs - whose existence is most difficult to explain by the classical model - are situated in wide binaries. This raises the possibility that these wide companions may play a role in initiating/maintaining the inward comet transport, e.g., by the Kozai-Lidov mechanism, or by launching a comet shower when an eccentric companion approaches an outer population of icy planetesimals. Both invoked mechanisms require time to be activated, and thus could explain the existence of older EDDs. However, further detailed anal- ysis is needed to assess whether these scenarios are able to explain all the observed features of EDDs. 


\section{ACKNOWLEDGMENTS}

The authors are grateful to the anonymous referee for the comments that improved the quality of this manuscript. This publication makes use of data products from the Wide-field Infrared Survey Explorer, which is a joint project of the University of California, Los Angeles, and the Jet Propulsion Laboratory/California Institute of Technology, and NEOWISE, which is a project of the Jet Propulsion Laboratory/California Institute of Technology. WISE and NEOWISE are funded by the National Aeronautics and Space Administration (NASA). This publication makes use of data products from the Two Micron All Sky Survey, which is a joint project of the University of Massachusetts and the Infrared Processing and Analysis Center/California Institute of Technology, funded by the National Aeronautics and Space Administration and the National Science Foundation. This work has made use of data from the European Space Agency (ESA) mission Gaia (https://www.cosmos.esa.int/gaia), processed by the Gaia Data Processing and Analysis Consortium (DPAC, https://www.cosmos.esa.int/web/ gaia/dpac/consortium). Funding for the DPAC has been provided by national institutions, in particular the institutions participating in the Gaia Multilateral Agreement. This research has made use of the NASA/ IPAC Infrared Science Archive, which is operated by the Jet Propulsion Laboratory, California Institute of Technology, under contract with the National Aeronautics and Space Administration. This research has also made use of the WEBDA database, operated at the Department of Theoretical Physics and Astrophysics of the Masaryk University We used the VizieR catalogue access tool and the Simbad object data base at CDS to gather data. Our work is partly based on observations obtained with the Apache Point Observatory 3.5-meter telescope, which is owned and operated by the Astrophysical Research Consortium. This project has been supported by the KH130526, K-125015, K-131508, K119517, and GINOP-2.3.2-15-2016-00003 grants of the National Research, Development and Innovation Office (NKFIH, Hungary) as well as the Lendület Program of the Hungarian Academy of Sciences, project No. LP2018-7/2019. Zs.M.Sz is supported by the ÚNKP-202 New National Excellence Program of the Ministry for Innovation and Technology from the source of the National Research, Development and Innovation Fund. AD was supported by the ÚNKP-20-5 New National Excellence Program of the Ministry for Innovation and Technology from the source of the National Research, Development amid Innovation Fund and the János Bolyai Research Scholarship of the Hungarian Academy of Sciences. A.D and Gy.M.Sz would like to thank the City of Szombathely for support under Agreement No. 67.17721/2016. G.C is supported by NAOJ ALMA Scientific Research Grant Number 2019-13B.
Facilities: Akari, IRAS, NEOWISE, Spitzer, TESS, WISE

Software: mpfit (Markwardt 2009), FITSH (Pál 2012), MUFRAN (Kollath 1990) 


\section{APPENDIX}

\section{A. CHECKING SOURCE CONFUSION}

While the emission is dominated by the stellar photosphere for all our sources in the W1 band, at longer wavelengths the contribution of excess increases (Table 4). In the W3 and W4 bands, the measured flux densities exceed the predicted photospheric contributions by at least $\sim 4.5$ and $\sim 8.7$ times, respectively. In the case of TYC 4209 , the observed excess is dominant even in the W2 band. If the excess is caused by a neighboring source(s), not resolved by WISE, then the position of the emitting source measured in W1 and in the other bands would be different. Another indication would be if the shape of the targets' profile does not match the typical profile of point sources. Examining the shape can also help to detect the effects of possible nearby nebulosity.

Since the AllWISE catalog does not include separate positional information for the different bands, we performed our analysis using the original WISE measurements. We utilized the "unWISE" coadds (Lang 2014) of the WISE all sky survey, which are designed to preserve the intrinsic resolution of the WISE images $\left(\sim 66^{\prime \prime} 1, \sim 6.6^{\prime \prime} 4, \sim 6\right.$ '. $^{\prime \prime}$, and $\sim 12$ '. 0 in the W1, W2, W3, and W4 bands, respectively). This provides a factor of $\sim 1.4$ narrower PSFs than those of the intentionally blurred "Atlas Image" products of the AllWISE Release. Figure 1 shows the vicinity of the six selected systems in all four WISE bands based on the unWISE data. We fitted two-dimensional Gaussians to the measured brightness profiles of our targets, determining their positions, the mean of the full width at half-maxima (FWHM) of the major and minor axes $\left(m_{A}\right)$, and the ratio of the FWHM of the minor to the major axis $\left(r_{A}\right)$. The same parameters were derived using the unWISE data of a comparison sample. For the comparison sample, we kept only those objects from the previously compiled AllWISE list (Sect. 2) for which the S/N of the flux measurement in each band is greater than the minimum $\mathrm{S} / \mathrm{N}$ for our six sources. This shortened list was also cross-matched with the TGAS catalog, but this time we did not remove giant stars and we made no cuts based on the parallax data, resulting in a database with 3732 stars.

For the six EDD candidates, the largest W2 and W3 band offsets compared to the W1 position were 0!'062 and 0 ". 415 . These do not count as outliers because $15 \%$ and $6 \%$ of the objects in the comparison sample display similar or larger offsets in the W2 and W3 bands, respectively. The W4 images of TYC 4946 and TYC 4479 show some extended nebulosity around the sources (in the case TYC 4479 this can already be noticed to some extent in the W3 image). This may explain that while for the other four objects the W4 offsets remain below $0{ }^{\prime \prime} 7$, for these two targets we measured offsets of $1^{\prime \prime} 1$. Nevertheless, even these latter values are consistent with those found for comparison objects with similar W4 band S/N (32\% and 27\% of them have larger offsets than that of TYC 4946 and TYC 4479, respectively). Neither the size nor the shape of the EDD candidates indicate significant contamination: we found that their $m_{A}$ and $r_{A}$ parameters fall within $2.3 \sigma$ of the comparison sample means in all four bands.

For three targets, additional higher spatial resolution mid-IR data are available. TYC 4479 was serendipitiously observed with the IRAC camera onboard Spitzer at $3.6 \mu \mathrm{m}$ and $4.5 \mu \mathrm{m}$ as part of a larger mapping project (Sect. 4.2), while TYC 4209 was the target of an extensive IRAC monitoring program at the same wavelengths (Moór et al. 2021, in preparation). Our targets appear as single sources in the obtained images that have three times better spatial resolution $\left(\mathrm{FWHM} \sim 2^{\prime \prime}\right)$ than that of WISE at the corresponding wavelengths. The recently released unWISE Catalog (Schlafly et al. 2019) - which considers all W1 and W2 band images obtained in the WISE and the first four year of the NEOWISE Reactivation missions - lists an object which is located 6".4 away from TYC 4515. This faint object ( $\sim 20 \times$ fainter than TYC 4515 in both bands) is actually an M-type companion of TYC 4515 (Sect. 3.3). However, the observed excess emission surely originates from the primary component. This is justified not only by the WISE observations, but by a $24 \mu \mathrm{m}$ Spitzer image where the position of the detected source coincides well with the optical position of TYC 4515 (Sect. 4.2). Thus, based on currently available IR data, we found no sign of confusion or significant contamination at our targets.

\section{B. ANALYSIS OF THE TESS DATA}

\section{B.1. Processing of TESS data}

Five of our stars were covered by the TESS spacecraft providing high quality 30 minute cadence photometric data. Table B1 shows the TESS Input Catalog (TIC) identifiers of our observed targets, as well as the log of their TESS measurements. We started the data reduction with the calibrated full-frame images which were downloaded from 
the MAST archive ${ }^{15}$. As a first step, following the outline given in Pál et al. (2020), we derived the plate solution using matched sources from the Gaia DR2 catalog. Considering the resemblance of the TESS throughput to that of Gaia $G_{\mathrm{RP}}$ (Ricker et al. 2015; Jordi et al. 2010), these matched objects were also utilized to compute a zero-point flux reference based on the $G_{\mathrm{RP}}$ magnitudes. By analyzing various TESS full-frame images, we found that the RMS of this flux calibration is $\sim 0.015$ mag. To extract the photometry of our sources, we performed differential image analysis utilizing the ficonv and fiphot tasks of the FITSH package (Pál 2012). The photometry was performed on $128 \times 128$ pixel subframes centered on the targets. The reference frame was constructed by computing a stray light-free median of 11 individual images measured close to the middle of the whole observing sequence. Lastly we identified and removed data points which were affected by momentum wheel desaturation or significant stray light (Pál et al. 2020). If the target was measured in more than one sector, then the above described procedure was carried out separately in each sector.

Table B1. TESS data

\begin{tabular}{c|ccccc}
\hline \hline Name & TIC ID & Sectors & Time range & $P_{\text {rot }}(\mathrm{d})$ & Amplitude (mag) \\
\hline TYC 4515 & TIC 142013492 & S19 & $11 / 27 / 2019-12 / 24 / 2019$ & 2.455 & 0.00026 \\
TYC 5940 & TIC 123977701 & S6 & $12 / 11 / 2018-01 / 07 / 2019$ & 3.756 & 0.0083 \\
TYC 8105 & TIC 231921033 & S5-7 & $11 / 15 / 2018-02 / 02 / 2019$ & $5.042 c$ & 0.0187 \\
TYC 4209 & TIC 233128866 & S15-17 & $08 / 15 / 2019-11 / 02 / 2019$ & 5.065 & 0.0032 \\
& & S19-20 & $11 / 27 / 2019-01 / 21 / 2020$ & & \\
& & S22-26 & $02 / 18 / 2020-07 / 04 / 2020$ & & \\
TYC 4479 & TIC 368374360 & S17-18 & $10 / 07 / 2019-11 / 27 / 2019$ & 32.08 & 0.0012 \\
& & S24-25 & $04 / 16 / 2020-06 / 08 / 2020$ & & \\
\hline
\end{tabular}

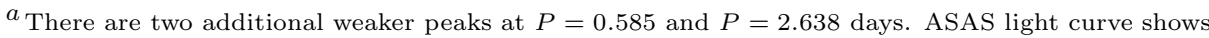
no convincing signals.

$b$ There are weaker Fourier-peaks at double/half frequencies. ASAS data show two close peaks $(P=$ 3.637 and $P=3.648$ ) confirming the findings from TESS observations.

${ }^{c}$ Two weaker peaks are found at $P=5.636$ and $P=2.509$ days. Some further irregular variations are still present after pre-whitening with these signals. ASAS light curve indicates one signal at $P=5.003$ days confirming the TESS analysis.

$d_{\text {There are additional nearby weak peaks, possibly due to differential rotation, and a signal at double }}$ frequency. ASAS light curve confirms the five-day signal $(P=5.126 d)$.

${ }^{e}$ Some weaker Fourier peaks with periods of $\approx 20$ days and amplitudes of $<50 \%$ that of the main signal are also present.

\section{B.2. Period analysis}

To identify periodic signals in the obtained TESS light curves, we performed a Fourier analysis with the MUlti FRequency ANalysis (MUFRAN) tool ${ }^{16}$ developed by Kollath (1990). Using MUFRAN, we prepared the discrete Fourier-transformation of the light curve for frequencies 0-10 1/days. We selected the most significant peaks, and after fitting the frequencies we pre-whitened the light curve, then iterated these steps until no more convincing Fourier peaks were found in the data. In many cases, longer trends (20-30 days) were found in the data. These might have astrophysical background or instrumental trends, but these cannot be confirmed with TESS data only, as their lengths are comparable to the observations themselves.

The TESS data showed periodic or quasi-periodic signals in all cases. In some targets, close to the main Fourier-peak additional smaller peaks were found: these are generally attributed to differential rotation of the stellar surface, i.e., regions of different latitudes showing slightly different rotational periods. Where available, we also checked light curves from the ASAS database to validate our findings from the TESS data. The derived main periods and amplitudes are summarized in Table B1.

\footnotetext{
15 https://mast.stsci.edu

16 http://www.konkoly.hu/staff/kollath/mufran.html
} 
Table C1. Stellar and disk properties of extreme debris systems identified prior to our study

\begin{tabular}{|c|c|c|c|c|c|c|c|c|c|c|c|c|}
\hline Name & (2) & $\begin{array}{c}\text { Dist. } \\
\text { (pc) } \\
(3)\end{array}$ & $\begin{array}{l}\text { Lum. } \\
\left(L_{\odot}\right) \\
(4) \\
\end{array}$ & $\begin{array}{l}T_{\text {eff }} \\
(\mathrm{K}) \\
(5) \\
\end{array}$ & Group & $\begin{array}{c}\text { Age } \\
(\mathrm{Myr}) \\
(7) \\
\end{array}$ & Mult. & $\begin{array}{l}T_{\mathrm{BB}} \\
(\mathrm{K}) \\
(9) \\
\end{array}$ & $\begin{array}{l}R_{\mathrm{BB}} \\
(\mathrm{au}) \\
(10) \\
\end{array}$ & (11) & Disk var. & (13) \\
\hline RZ Psc & KoIV & 184.7 & 1.0 & 5350 & - & $30_{-10}^{+10}$ & Y & $340-500$ & $0.3-0.7$ & $0.049-0.08$ & $\mathrm{Y}(\mathrm{W})$ & $9,17,21,35,37,38$ \\
\hline $\mathrm{BD}+20307$ & F9V & 116.9 & 2.8 & 6000 & - & $\gtrsim 1000$ & Y & $358-440$ & $0.7-0.9$ & $0.01-0.04$ & $\mathrm{Y}(\mathrm{S})$ & $5,11,16,19,29,43,48,49,50,51,52,53$ \\
\hline HD 15407 & F5V & 49.3 & 3.25 & 6500 & ABDor & $149_{-19}^{+51}$ & Y & $500-1020$ & $0.13-0.6$ & $0.006-0.0097$ & $\mathrm{Y}(\mathrm{S})$ & $3,26,29,32,49$ \\
\hline V488 Per & $\mathrm{K} 3 \mathrm{~V}$ & 172.9 & 0.3 & 4900 & $\alpha$ Per & $85_{-10}^{+10}$ & Y? & 820 & 0.06 & 0.16 & $\mathrm{Y}(\mathrm{W})$ & $2,8,24,54,55$ \\
\hline HD 23514 & F5V & 139.1 & 3.0 & 6450 & Pleiades & $125_{-8}^{+8}$ & $\mathrm{Y}$ & $600-1080$ & $0.1-0.4$ & 0.02 & $\mathrm{Y}(\mathrm{S}, \mathrm{W})$ & $7,29,39,40,44,49$ \\
\hline P 1121 & F9V & 440.4 & 1.6 & 6050 & NGC 2422 & $140_{-20}^{+20}$ & $\mathrm{~N}$ & 460 & 0.5 & $0.014-0.020$ & $\mathrm{Y}(\mathrm{S}, \mathrm{W})$ & $1,5,6,13,29,45$ \\
\hline ID 8 & G6V & 355.7 & 0.7 & 5500 & NGC 2547 & $35_{-4}^{+4}$ & $\mathrm{~N}$ & 400 & 0.4 & $0.025-0.032$ & $\mathrm{Y}(\mathrm{S}, \mathrm{W})$ & $14,18,28,30,31,45$ \\
\hline TYC 8241-2652-1 $a$ & $\mathrm{~K} 2 \mathrm{~V}$ & 121.3 & 0.6 & 4950 & LCC & $15_{-3}^{+3}$ & $\mathrm{~N}$ & 450 & 0.3 & 0.11 & $\mathrm{Y}(\mathrm{O}, \mathrm{W})$ & $15,27,34$ \\
\hline HD 113766 & $\mathrm{~F} 2 \mathrm{~V}$ & 108.6 & 3.9 & 6800 & $\mathrm{LCC}$ & $15_{-3}^{+3}$ & Y & 490 & 0.64 & 0.017 & $\mathrm{Y}(\mathrm{S})$ & $4,10,22,32,33,47$ \\
\hline HD 145263 & $\mathrm{~F} 2 \mathrm{~V}$ & 141.2 & 4.5 & 6800 & US & $10_{-3}^{+3}$ & $\mathrm{~N}$ & $240-290$ & $2.0-2.9$ & $0.01-0.02$ & $\mathrm{~N}(\mathrm{~S}, \mathrm{~W})$ & $10,12,19,23,25,30,33,42$ \\
\hline HD 166191 & G0 & 100.8 & 4.0 & 6000 & HD $166191^{b}$ & $10_{-5}^{+5}$ & $\mathrm{~N}$ & 760 & 0.3 & 0.06 & $\mathrm{Y}(\mathrm{S})$ & $20,32,36,41,46$ \\
\hline
\end{tabular}

Note- Column (1): Name of the star. Column (2): Spectral type. Column (3): Distance (Bailer-Jones et al. 2020). Column (4): Luminosity. Literature data are scaled corresponding to the new distances from Col. 3 when needed. Column (5): Effective temperature. Column (6): Group membership. ABDor: AB Doradus moving group; LCC: Lower Centaurus Crux association; US: Upper Scorpius association. Column (7): Stellar age. In the case of group members, age of the corresponding group is quoted. Column (8): Multiplicity. See Sect. 6.3.2 for more details. Column (9): Dust temperature or temperature range when several different estimates are available in the literature. In cases where the SED was fitted with two temperature components, the warmer one is considered. Column (10): Disk radius. For comparability with our results presented in Table 1 we quote the blackbody radii that were computed using luminosities and dust temperatures from Col. (4) and Col. (9). For possible caveats related to this approach, see Sect. 4.3. Column (11): Fractional luminosity. If this is a range, then it shows the scatter of the literature data and is not because of variability. Column (12): Disk variability in the wavelength regime between 3 and $5 \mu \mathrm{m}$. Entries in parentheses show what type of measurements were used to examine the variability - S: Spitzer photometry; W: WISE photometry; G: ground based instruments. Column (13): References: (1) Gaia Collaboration et al. (2018a); (2) Barrado y Navascués et al. (2004); (3) Bell et al. (2015); (4) Chen et al. (2006); (5) Cotten \& Song (2016); (6) Cummings \& Kalirai (2018); (7) David \& Hillenbrand (2015); (8) Deacon \& Kraus (2020); (9) de Wit et al. (2013); (10) de Zeeuw et al. (1999); (11) Fekel et al. (2012); (12) Fujiwara et al. (2013); (13) Gorlova et al. (2004); (14) Gorlova et al. (2007); (15) Günther et al. (2017); (16) Hartman \& Lépine (2020); (17) Herbig (1960); (18) Jeffries \& Oliveira (2005); (19) Kennedy \& Wyatt (2013); (20) Kennedy \& Wyatt (2014); (21) Kennedy et al. (2020); (22) Lisse et al. (2017); (23) Lisse et al. (2020); (24) Luo et al. (2019); (25) Mannings \& Barlow (1998); (26) Melis et al. (2010); (27) Melis et al. (2012); (28) Meng et al. 2014); (29) Meng et al. (2015); (30) Mittal et al. (2015); (31) Olofsson et al. (2012); (32) Oudmaijer et al. (1992); (33) Pecaut et al. (2012); (34) Pecaut \& Mamajek (2016); 35) Potravnov et al. (2014); (36) Potravnov et al. (2018); (37) Potravnov et al. (2019); (38) Punzi et al. (2018); (39) Rhee et al. (2008); (40) Rodriguez et al. (2012); (41) Schneider et al. (2013); (42) Smith et al. (2008); (43) Song et al. (2005); (44) Stauffer et al. (1998); (45) Su et al. (2019b); (46) Su et al. (2019a); (47) Su et al. (2020); (48) Thompson et al. (2019); (49) Vican et al. (2016); (50) Weinberger (2008); (51) Weinberger et al. (2011); (52) Whitelock et al. (1991); (53) Zuckerman et al. (2008); (54) Zuckerman et al. (2012); (55) Zuckerman (2015)

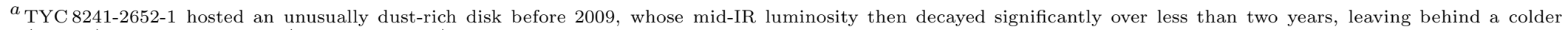
$(<200 \mathrm{~K})$, more tenuous disk (Melis et al. 2012). The quoted data show the disk parameters measured before 2009.

${ }^{b}$ Kennedy \& Wyatt (2014) found that HD 166191 is comoving with the well known Herbig Ae star, HD 163296. Later, Potravnov et al. (2018) identified some additional young stars showing similar kinematics and located close to HD 166191 and they argued that all these objects belong to a $\sim 10$ Myr old kinematic group.

\section{PROPERTIES AND LONG TERM MID-INFRARED VARIABILITY OF PREVIOUSLY KNOWN EDDS}

Prior to our study, eleven $\mathrm{F}-\mathrm{K}$ type stars were reported to host extreme debris disks. In Table $\mathrm{C} 1$ we summarize the fundamental properties of these systems.

Using the available single-exposure photometry acquired in the W1 and W2 bands over the different WISE mission phases, we explored the long term infrared variability of the 11 previously known EDDs listed in Table C1. Some of these targets are brighter than 8 and 7 mags in the W1 and W2 bands, respectively, and thus are potentially affected by non-linearity and saturation effects. As Mainzer et al. (2014) showed for sources brighter than the saturation limits, fluxes measured in the reactivated NEOWISE mission could be significantly overestimated relative to the AllWISE mission data (see their fig. 6). Therefore, our previously used quality criteria (Sect. 4.1) were supplemented with an additional one: we ignored those data points where the fraction of the saturated pixels in the profile-fitting area exceeds 0.05 (w1sat>0.05, w2sat>0.05). For the three brightest sources, HD 15407, HD 113766, and HD 166191, this criterion removes essentially all data points. In the cases of BD+20 307 and HD 145263, the W1 band data turned out to be heavily saturated, but we could extract the light curve in the W2 band. From the rest of the sample, only the W1 photometry of HD 23514 was somewhat affected by this issue, but it did not prevent the compilation of the light curve. The photometry of ID8 has a different quality issue: the reduced $\chi^{2}$ of all of its W1 band profile-fit photometry measurements (w1rchi2) obtained during the AllWISE mission phase are higher than the critical value we defined $(>5$, Sect. 4.1). However, looking at the specific observation windows, the standard deviation of the points did not 
appear to be substantially higher than in the other windows and therefore we have waived the application of this quality criterion.

In order to assess the long term variability of the targets, we used the same strategy as described in Sect. 5.1. For BD+20 307 and HD 145263, we applied only the $\chi^{2}$ test and found both objects to be constant within the errors of the $4.6 \mu \mathrm{m}$ observations. The mid-IR flux of the disk around TYC 8241-2652-1 dropped significantly before the start of the WISE mission (Melis et al. 2012) and recent observations indicated that the disk has remained depleted (Günther et al. 2017). In the W1/W2 bands, the source does not exhibit significant excess, thus it is not surprising that we found no variability in either filter. The other five objects (ID 8, P 1121, V488 Per, RZ Psc, and HD 23514), however, proved to be variable at both 3.4 and $4.6 \mu \mathrm{m}$. Apart from HD 23514, significant color (W1-W2) variations were also detected. Figure $\mathrm{C} 1$ displays their average disk flux densities in each observational window, typically separated by $\sim 6$ months, between 2010 and 2019. To compute these data, we subtracted the photospheric contributions from the measured flux densities. For photosphere models, the stellar parameters were taken from the literature and the corresponding ATLAS9 model was fitted to the available optical and near-infrared photometric data of the given source. The bottom panels of each plot show the ratio of W1/W2 disk fluxes and the corresponding color temperatures. 

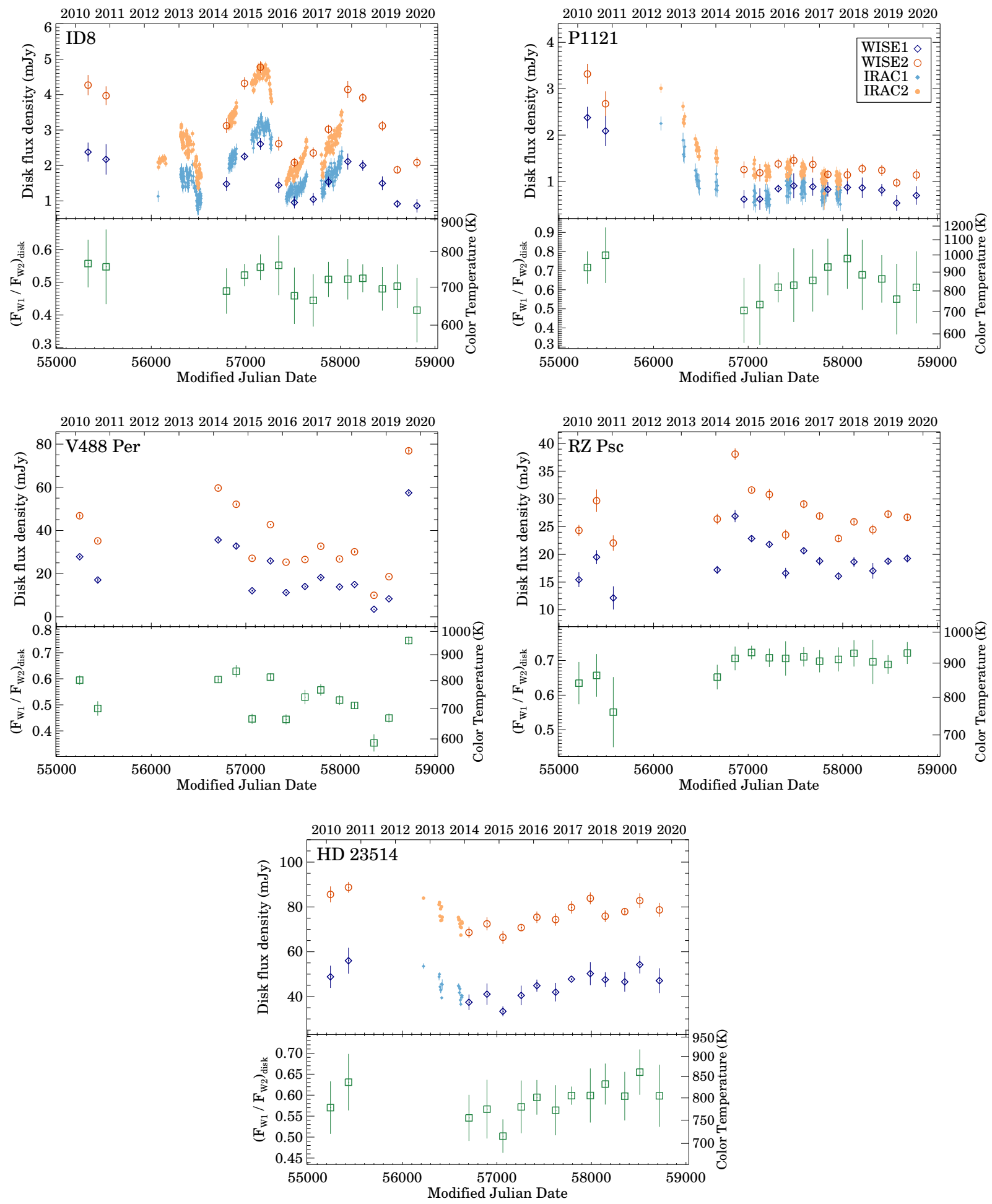

Figure C1. Seasonal averages of W1 and W2 band disk fluxes (top panels) and their ratios (bottom panels) between 2010 and 2019 for five previously known EDDs. Disk fluxes derived from multi-year Spitzer photometric monitoring of HD 23514, ID 8, and P 1121 (Meng et al. 2015; Su et al. 2019b) are also plotted with smaller filled symbols. 


\section{REFERENCES}

Abbott, T. M. C., Abdalla, F. B., Allam, S., et al. 2018, ApJS, 239, 18

Agüeros, M. A., Bowsher, E. C., Bochanski, J. J., et al. 2018, ApJ, 862, 33

Alam, S., Albareti, F. D., Allende Prieto, C., et al. 2015, ApJS, 219, 12

Almeida-Fernandes, F., \& Rocha-Pinto, H. J. 2018, MNRAS, 480, 4903

Amard, L., \& Matt, S. P. 2020, ApJ, 889, 108

Andrews, J. J., Chanamé, J., \& Agüeros, M. A. 2017, MNRAS, 472, 675

Angus, R., Aigrain, S., Foreman-Mackey, D., \& McQuillan, A. 2015, MNRAS, 450, 1787

Bailer-Jones, C. A. L., Rybizki, J., Fouesneau, M., Demleitner, M., \& Andrae, R. 2020, arXiv e-prints, arXiv:2012.05220

Balachandran, S. 1995, ApJ, 446, 203

Ballering, N. P., Rieke, G. H., Su, K. Y. L., \& Gáspár, A. 2017, ApJ, 845, 120

Balog, Z., Kiss, L. L., Vinkó, J., et al. 2009, ApJ, 698, 1989

Baraffe, I., Homeier, D., Allard, F., \& Chabrier, G. 2015, A\&A, 577, A42

Barclay, T., Quintana, E. V., Raymond, S. N., \& Penny, M. T. 2017, ApJ, 841, 86

Barnes, S. A. 2003, ApJ, 586, 464

—. 2007, ApJ, 669, 1167

Barnes, S. A., Weingrill, J., Fritzewski, D., Strassmeier, K. G., \& Platais, I. 2016, ApJ, 823, 16

Barrado y Navascués, D., Stauffer, J. R., \& Jayawardhana, R. 2004, ApJ, 614, 386

Beichman, C. A., Lisse, C. M., Tanner, A. M., et al. 2011, ApJ, 743, 85

Bell, C. P. M., Mamajek, E. E., \& Naylor, T. 2015, MNRAS, 454, 593

Berski, F., \& Dybczyński, P. A. 2016, A\&A, 595, L10

Beust, H., Vidal-Madjar, A., \& Ferlet, R. 1991, A\&A, 247, 505

Blanco-Cuaresma, S., Soubiran, C., Heiter, U., \& Jofré, P. 2014, A\&A, 569, A111

Boeche, C., \& Grebel, E. K. 2016, A\&A, 587, A2

Boesgaard, A. M., \& Tripicco, M. J. 1986, ApJL, 302, L49

Bonsor, A., Augereau, J. C., \& Thébault, P. 2012, A\&A, 548, A104

Bonsor, A., Raymond, S. N., \& Augereau, J.-C. 2013, MNRAS, 433, 2938

Bonsor, A., Raymond, S. N., Augereau, J.-C., \& Ormel, C. W. 2014, MNRAS, 441, 2380

Bonsor, A., \& Veras, D. 2015, MNRAS, 454, 53
Booth, M., Wyatt, M. C., Morbidelli, A., Moro-Martín, A., \& Levison, H. F. 2009, MNRAS, 399, 385

Bossini, D., Vallenari, A., Bragaglia, A., et al. 2019, A\&A, 623, A108

Bottke, W. F., \& Norman, M. D. 2017, Annual Review of Earth and Planetary Sciences, 45, 619

Brandeker, A., \& Cataldi, G. 2019, A\&A, 621, A86

Bressan, A., Marigo, P., Girardi, L., et al. 2012, MNRAS, 427,127

Burgasser, A. J., Kirkpatrick, J. D., Reid, I. N., et al. 2003, ApJ, 586, 512

Canup, R. M., \& Asphaug, E. 2001, Nature, 412, 708

Cardelli, J. A., Clayton, G. C., \& Mathis, J. S. 1989, ApJ, 345,245

Cargile, P. A., James, D. J., \& Jeffries, R. D. 2010, ApJL, 725, L111

Carpenter, J. M., Bouwman, J., Mamajek, E. E., et al. 2009, ApJS, 181, 197

Castelli, F., \& Kurucz, R. L. 2004, ArXiv Astrophysics e-prints, astro-ph/0405087

Chambers, J. E. 2013, Icarus, 224, 43

Chambers, K. C., Magnier, E. A., Metcalfe, N., et al. 2016, arXiv e-prints, arXiv:1612.05560

Chen, C. H., Mittal, T., Kuchner, M., et al. 2014, ApJS, 211,25

Chen, C. H., Sargent, B. A., Bohac, C., et al. 2006, ApJS, 166,351

Cotten, T. H., \& Song, I. 2016, ApJS, 225, 15

Cruz-Saenz de Miera, F., Chavez, M., Bertone, E., \& Vega, O. 2014, MNRAS, 437, 391

Cummings, J. D., \& Kalirai, J. S. 2018, AJ, 156, 165

Cutri, R. M., \& et al. 2013, VizieR Online Data Catalog, II $/ 328$

Cutri, R. M., Skrutskie, M. F., van Dyk, S., et al. 2003, VizieR Online Data Catalog, 2246

da Silva, L., Torres, C. A. O., de La Reza, R., et al. 2009, A\&A, 508, 833

Dahm, S. E. 2015, ApJ, 813, 108

Daniel, S. A., Latham, D. W., Mathieu, R. D., \& Twarog, B. A. 1994, PASP, 106, 281

David, T. J., \& Hillenbrand, L. A. 2015, ApJ, 804, 146

Davis, M., Hut, P., \& Muller, R. A. 1984, Nature, 308, 715 de Wit, W. J., Grinin, V. P., Potravnov, I. S., et al. 2013, A\&A, 553, L1

de Zeeuw, P. T., Hoogerwerf, R., de Bruijne, J. H. J., Brown, A. G. A., \& Blaauw, A. 1999, AJ, 117, 354

Deacon, N. R., \& Kraus, A. L. 2020, MNRAS, 496, 5176

Dobbie, P. D., Lodieu, N., \& Sharp, R. G. 2010, MNRAS, 409, 1002 
Douglas, S. T., Curtis, J. L., Agüeros, M. A., et al. 2019, ApJ, 879, 100

Duchêne, G., \& Kraus, A. 2013, ARA\&A, 51, 269

Elliott, P., Huélamo, N., Bouy, H., et al. 2015, A\&A, 580, A88

Ertel, S., Defrère, D., Hinz, P., et al. 2020, AJ, 159, 177

Fabricius, C., Luri, X., Arenou, F., et al. 2020, arXiv e-prints, arXiv:2012.06242

Faramaz, V., Ertel, S., Booth, M., Cuadra, J., \& Simmonds, C. 2017, MNRAS, 465, 2352

Faramaz, V., Krist, J., Stapelfeldt, K. R., et al. 2019, AJ, 158,162

Fekel, F. C., Cordero, M. J., Galicher, R., et al. 2012, ApJ, 749,7

Fischer, D. A., \& Marcy, G. W. 1992, ApJ, 396, 178

Flaherty, K., Hughes, A. M., Mamajek, E. E., \& Murphy, S. J. 2019, ApJ, 872, 92

Fouesneau, M., Rix, H.-W., von Hippel, T., Hogg, D. W., \& Tian, H. 2019, ApJ, 870, 9

Frandsen, S., \& Lindberg, B. 1999, in Astrophysics with the NOT, ed. H. Karttunen \& V. Piirola, Piikkio, Finland : University of Turku, Tuorla Observatory, 1999, p.71

Fujiwara, H., Ishihara, D., Onaka, T., et al. 2013, A\&A, 550, A45

Gagné, J., Mamajek, E. E., Malo, L., et al. 2018, ApJ, 856, 23

Gaia Collaboration, Brown, A. G. A., Vallenari, A., et al. 2020, arXiv e-prints, arXiv:2012.01533

Gaia Collaboration, Babusiaux, C., van Leeuwen, F., et al. 2018a, A\&A, 616, A10

Gaia Collaboration, Brown, A. G. A., Vallenari, A., et al. 2018b, A\&A, 616, A1

Garcia, D. E., \& Hughes, A. M. 2019, in American Astronomical Society Meeting Abstracts, Vol. 233, American Astronomical Society Meeting Abstracts \#233, 163.02

Geiler, F., \& Krivov, A. V. 2017, MNRAS, 468, 959

Genda, H., Kobayashi, H., \& Kokubo, E. 2015, ApJ, 810, 136

Gentile Fusillo, N. P., Tremblay, P.-E., Gänsicke, B. T., et al. 2019, MNRAS, 482, 4570

Giampapa, M. S., Hall, J. C., Radick, R. R., \& Baliunas, S. L. 2006, ApJ, 651, 444

Gomes, R., Levison, H. F., Tsiganis, K., \& Morbidelli, A. 2005, Nature, 435, 466

Gorlova, N., Balog, Z., Rieke, G. H., et al. 2007, ApJ, 670, 516

Gorlova, N., Padgett, D. L., Rieke, G. H., et al. 2004, ApJS, 154, 448
Günther, H. M., Kraus, S., Melis, C., et al. 2017, A\&A, 598, A82

Halliday, A. N. 2008, Philosophical Transactions of the Royal Society of London Series A, 366, 4163

Hartman, Z. D., \& Lépine, S. 2020, ApJS, 247, 66

Helou, G., Khan, I. R., Malek, L., \& Boehmer, L. 1988, ApJS, 68, 151

Henden, A. A., Templeton, M., Terrell, D., et al. 2016, VizieR Online Data Catalog, 2336

Herbig, G. H. 1960, ApJ, 131, 632

Høg, E., Fabricius, C., Makarov, V. V., et al. 2000, A\&A, 357,367

Hughes, A. M., Duchêne, G., \& Matthews, B. C. 2018, ARA\&A, 56, 541

Iglesias, D., Bayo, A., Olofsson, J., et al. 2018, MNRAS, 480,488

Ishihara, D., Onaka, T., Kataza, H., et al. 2010, A\&A, 514, A1

Jackson, A. P., \& Wyatt, M. C. 2012, MNRAS, 425, 657

Jacobsen, S. B. 2005, Annual Review of Earth and Planetary Sciences, 33, 531

Jeffries, R. D., \& Oliveira, J. M. 2005, MNRAS, 358, 13

Jiang, Y.-F., \& Tremaine, S. 2010, MNRAS, 401, 977

Jordi, C., Gebran, M., Carrasco, J. M., et al. 2010, A\&A, 523, A48

Juarez, A. J., Cargile, P. A., James, D. J., \& Stassun, K. G. 2014, ApJ, 795, 143

Kaib, N. A., Raymond, S. N., \& Duncan, M. 2013, Nature, 493, 381

Kaufer, A., Stahl, O., Tubbesing, S., et al. 1999, The Messenger, 95, 8

Kawaler, S. D. 1988, ApJ, 333, 236

Kennedy, G. M., Kenworthy, M. A., Pepper, J., et al. 2017, Royal Society Open Science, 4, 160652

Kennedy, G. M., \& Piette, A. 2015, MNRAS, 449, 2304

Kennedy, G. M., \& Wyatt, M. C. 2013, MNRAS, 433, 2334 —. 2014, MNRAS, 444, 3164

Kennedy, G. M., Ginski, C., Kenworthy, M. A., et al. 2020, MNRAS, 496, L75

Kiefer, F., Lecavelier des Etangs, A., Augereau, J. C., et al. 2014, A\&A, 561, L10

Kleine, T., Touboul, M., Bourdon, B., et al. 2009, GeoCoA, 73,5150

Kochanek, C. S., Shappee, B. J., Stanek, K. Z., et al. 2017, PASP, 129, 104502

Kollath, Z. 1990, Konkoly Observatory Occasional Technical Notes, 1, 1

Kraft, R. P. 1967, ApJ, 150, 551

Krivov, A. V. 2010, Research in Astronomy and Astrophysics, 10, 383 
Lada, C. J., Muench, A. A., Rathborne, J., Alves, J. F., \& Lombardi, M. 2008, ApJ, 672, 410

Lang, D. 2014, AJ, 147, 108

Lindegren, L., Lammers, U., Bastian, U., et al. 2016, A\&A, 595, A4

Lindegren, L., Klioner, S. A., Hernández, J., et al. 2020, arXiv e-prints, arXiv:2012.03380

Lisse, C. M., Sitko, M. L., Russell, R. W., et al. 2017, ApJL, 840, L20

Lisse, C. M., Meng, H. Y. A., Sitko, M. L., et al. 2020, ApJ, 894,116

Löhne, T., Augereau, J. C., Ertel, S., et al. 2012, A\&A, 537, A110

Luo, A. L., Zhao, Y. H., Zhao, G., \& et al. 2019, VizieR Online Data Catalog, V/164

Mainzer, A., Bauer, J., Cutri, R. M., et al. 2014, ApJ, 792, 30

Makovoz, D., \& Marleau, F. R. 2005, PASP, 117, 1113

Mamajek, E. E., \& Hillenbrand, L. A. 2008, ApJ, 687, 1264

Mamajek, E. E., Meyer, M. R., \& Liebert, J. 2002, AJ, 124, 1670

Mann, A. W., Feiden, G. A., Gaidos, E., Boyajian, T., \& von Braun, K. 2015, ApJ, 804, 64

Mannings, V., \& Barlow, M. J. 1998, ApJ, 497, 330

Marboeuf, U., Bonsor, A., \& Augereau, J. C. 2016, Planet. Space Sci., 133, 47

Marino, S., Bonsor, A., Wyatt, M. C., \& Kral, Q. 2018, MNRAS, 479, 1651

Marino, S., Wyatt, M. C., Panić, O., et al. 2017, MNRAS, 465,2595

Markwardt, C. B. 2009, in Astronomical Society of the Pacific Conference Series, Vol. 411, Astronomical Data Analysis Software and Systems XVIII, ed. D. A. Bohlender, D. Durand, \& P. Dowler, 251

Meibom, S., Mathieu, R. D., \& Stassun, K. G. 2009, ApJ, 695,679

Melis, C., Zuckerman, B., Rhee, J. H., \& Song, I. 2010, ApJL, 717, L57

Melis, C., Zuckerman, B., Rhee, J. H., et al. 2012, Nature, 487, 74

Meng, H. Y. A., Rieke, G. H., Su, K. Y. L., et al. 2012, ApJL, 751, L17

Meng, H. Y. A., Su, K. Y. L., Rieke, G. H., et al. 2014, Science, 345, 1032

Meng, H. Y. A., Su, K. Y. L., Rieke, G. H., et al. 2015, ApJ, 805, 77

Michalik, D., Lindegren, L., \& Hobbs, D. 2015, A\&A, 574, A115

Mittal, T., Chen, C. H., Jang-Condell, H., et al. 2015, ApJ, 798,87
Montesinos, B., Eiroa, C., Krivov, A. V., et al. 2016, A\&A, 593, A51

Morales, F. Y., Rieke, G. H., Werner, M. W., et al. 2011, ApJL, 730, L29

Moshir, M. e. 1990, in IRAS Faint Source Catalogue, version 2.0 (1990)

Murray, C. D., \& Dermott, S. F. 1999, Solar system dynamics (Cambridge, UK: Cambridge University Press)

Naoz, S. 2016, ARA\&A, 54, 441

Nesvold, E. R., Naoz, S., Vican, L., \& Farr, W. M. 2016, ApJ, 826, 19

Nesvorný, D., Jenniskens, P., Levison, H. F., et al. 2010, ApJ, 713, 816

Neugebauer, G., Habing, H. J., van Duinen, R., et al. 1984, ApJL, 278, L1

Olofsson, J., Juhász, A., Henning, T., et al. 2012, A\&A, 542, A90

Oudmaijer, R. D., van der Veen, W. E. C. J., Waters, L. B. F. M., et al. 1992, A\&AS, 96, 625

Pál, A. 2012, MNRAS, 421, 1825

Pál, A., Szakáts, R., Kiss, C., et al. 2020, ApJS, 247, 26

Pascucci, I., Testi, L., Herczeg, G. J., et al. 2016, ApJ, 831, 125

Patel, R. I., Metchev, S. A., \& Heinze, A. 2014, ApJS, 212, 10

Patel, R. I., Metchev, S. A., Heinze, A., \& Trollo, J. 2017, AJ, 153, 54

Pawellek, N., Krivov, A. V., Marshall, J. P., et al. 2014, ApJ, 792, 65

Pecaut, M. J., \& Mamajek, E. E. 2013, ApJS, 208, 9

—. 2016, MNRAS, 461, 794

Pecaut, M. J., Mamajek, E. E., \& Bubar, E. J. 2012, ApJ, 746,154

Pilbratt, G. L., Riedinger, J. R., Passvogel, T., et al. 2010, A\&A, 518, L1

Potravnov, I. S., Eselevich, M. V., Kondratieva, T. E., \& Sokolov, I. V. 2018, Astronomy Letters, 44, 603

Potravnov, I. S., Grinin, V. P., Ilyin, I. V., \& Shakhovskoy, D. N. 2014, A\&A, 563, A139

Potravnov, I. S., Grinin, V. P., \& Serebriakova, N. A. 2019, A\&A, 630, A64

Potravnov, I. S., Mkrtichian, D. E., Grinin, V. P., Ilyin, I. V., \& Shakhovskoy, D. N. 2017, A\&A, 599, A60

Punzi, K. M., Kastner, J. H., Melis, C., et al. 2018, AJ, 155,33

Qian, S.-B., Shi, X.-D., Zhu, L.-Y., et al. 2019, Research in Astronomy and Astrophysics, 19, 064

Quintana, E. V., Barclay, T., Borucki, W. J., Rowe, J. F., \& Chambers, J. E. 2016, ApJ, 821, 126 
Raghavan, D., McAlister, H. A., Henry, T. J., et al. 2010, ApJS, 190, 1

Rebollido, I., Eiroa, C., Montesinos, B., et al. 2020, A\&A, 639, A11

Rebull, L. M., Stauffer, J. R., Bouvier, J., et al. 2016, AJ, 152, 113

Reidemeister, M., Krivov, A. V., Stark, C. C., et al. 2011, A\&A, 527, A57

Rhee, J. H., Song, I., \& Zuckerman, B. 2008, ApJ, 675, 777

Rhee, J. H., Song, I., Zuckerman, B., \& McElwain, M. 2007, ApJ, 660, 1556

Ricker, G. R., Winn, J. N., Vanderspek, R., et al. 2015, Journal of Astronomical Telescopes, Instruments, and Systems, 1, 014003

Rieke, G. H., Young, E. T., Engelbracht, C. W., et al. 2004, ApJS, 154, 25

Riello, M., De Angeli, F., Evans, D. W., et al. 2020, arXiv e-prints, arXiv:2012.01916

Rigley, J. K., \& Wyatt, M. C. 2020, MNRAS, 497, 1143

Rodriguez, D. R., Marois, C., Zuckerman, B., Macintosh, B., \& Melis, C. 2012, ApJ, 748, 30

Rowan-Robinson, M., \& May, B. 2013, MNRAS, 429, 2894

Sarajedini, A., Dotter, A., \& Kirkpatrick, A. 2009, ApJ, 698, 1872

Schatzman, E. 1962, Annales d'Astrophysique, 25, 18

Schlafly, E. F., Meisner, A. M., \& Green, G. M. 2019, ApJS, 240, 30

Schneider, A., Song, I., Melis, C., et al. 2013, ApJ, 777, 78

Secrest, N. J., \& Satyapal, S. 2020, ApJ, 900, 56

Sepulveda, A. G., Matrà, L., Kennedy, G. M., et al. 2019, ApJ, 881, 84

Sestito, P., \& Randich, S. 2005, A\&A, 442, 615

Sezestre, É., Augereau, J. C., \& Thébault, P. 2019, A\&A, 626, A2

Shappee, B. J., Prieto, J. L., Grupe, D., et al. 2014, ApJ, 788,48

Sibthorpe, B., Kennedy, G. M., Wyatt, M. C., et al. 2018, MNRAS, 475, 3046

Silverberg, S. M., Wisniewski, J. P., Kuchner, M. J., et al. 2020, ApJ, 890, 106

Silverstone, M. D., Meyer, M. R., Mamajek, E. E., et al. 2006, ApJ, 639, 1138

Skrutskie, M. F., Cutri, R. M., Stiening, R., et al. 2006, AJ, 131, 1163

Smith, R., Wyatt, M. C., \& Dent, W. R. F. 2008, A\&A, 485,897

Sneden, C. A. 1973, PhD thesis, THE UNIVERSITY OF TEXAS AT AUSTIN.

Soderblom, D. R., Jones, B. F., Balachandran, S., et al. 1993, AJ, 106, 1059
Sokolovsky, K. V., Gavras, P., Karampelas, A., et al. 2017, MNRAS, 464, 274

Song, I., Zuckerman, B., Weinberger, A. J., \& Becklin, E. E. 2005, Nature, 436, 363

Soubiran, C., Cantat-Gaudin, T., Romero-Gómez, M., et al. 2018, A\&A, 619, A155

Stauffer, J., Rebull, L., Bouvier, J., et al. 2016, AJ, 152, 115

Stauffer, J. R., Schultz, G., \& Kirkpatrick, J. D. 1998, ApJL, 499, L199

Steinhauer, A., \& Deliyannis, C. P. 2004, ApJL, 614, L65

Stetson, P. B. 1996, PASP, 108, 851

Su, K., Rieke, G., \& Sand, D. 2019a, The Astronomer's Telegram, 12986, 1

Su, K. Y. L., Rieke, G. H., Melis, C., et al. 2020, ApJ, 898, 21

Su, K. Y. L., Rieke, G. H., Stansberry, J. A., et al. 2006, ApJ, 653, 675

Su, K. Y. L., Jackson, A. P., Gáspár, A., et al. 2019b, AJ, 157,202

Telting, J. H., Avila, G., Buchhave, L., et al. 2014, Astronomische Nachrichten, 335, 41

Tera, F., Papanastassiou, D. A., \& Wasserburg, G. J. 1974, Earth and Planetary Science Letters, 22, 1

Thompson, M. A., Weinberger, A. J., Keller, L. D., Arnold, J. A., \& Stark, C. C. 2019, ApJ, 875, 45

Thureau, N. D., Greaves, J. S., Matthews, B. C., et al. 2014, MNRAS, 445, 2558

Tokovinin, A., \& Briceño, C. 2020, AJ, 159, 15

Tonry, J. L., Stubbs, C. W., Lykke, K. R., et al. 2012, ApJ, 750,99

Touboul, M., Kleine, T., Bourdon, B., Palme, H., \& Wieler, R. 2007, Nature, 450, 1206

Twarog, B. A., Anthony-Twarog, B. J., Deliyannis, C. P., \& Thomas, D. T. 2015, AJ, 150, 134

VandenBerg, D. A., \& Stetson, P. B. 2004, PASP, 116, 997

Vican, L., Schneider, A., Bryden, G., et al. 2016, ApJ, 833, 263

Villanova, S., Carraro, G., \& Saviane, I. 2009, A\&A, 504, 845

Weinberg, M. D., Shapiro, S. L., \& Wasserman, I. 1987, ApJ, 312, 367

Weinberger, A. J. 2008, ApJL, 679, L41

Weinberger, A. J., Becklin, E. E., Song, I., \& Zuckerman, B. 2011, ApJ, 726, 72

Welsh, B. Y., \& Montgomery, S. L. 2015, Advances in Astronomy, 2015, 980323

Werner, M. W., Roellig, T. L., Low, F. J., et al. 2004, ApJS, 154, 1

Whitelock, P. A., Menzies, J. W., Catchpole, R. M., et al. 1991, MNRAS, 250, 638 
Wright, E. L., Eisenhardt, P. R. M., Mainzer, A. K., et al. 2010, AJ, 140, 1868

Wyatt, M. C. 2008, ARA\&A, 46, 339

Wyatt, M. C., Smith, R., Greaves, J. S., et al. 2007, ApJ, 658,569

Zuckerman, B. 2015, ApJ, 798, 86
Zuckerman, B., Fekel, F. C., Williamson, M. H., Henry, G. W., \& Muno, M. P. 2008, ApJ, 688, 1345

Zuckerman, B., Klein, B., \& Kastner, J. 2019, ApJ, 887, 87

Zuckerman, B., Melis, C., Rhee, J. H., Schneider, A., \& Song, I. 2012, ApJ, 752, 58 